\title{
An Enhanced Measurement of the Angular Response of Photomultiplier Tubes at the Sudbury Neutrino Observatory
}

Olivier Simard, B. Sc.

\author{
A thesis submitted to the \\ Faculty of Graduate Studies and Research \\ in partial fulfillment of the requirements \\ for the degree of \\ Master of Sciences
}

Ottawa-Carleton Institute for Physics

Department of Physics

Carleton University

Ottawa, Ontario, Canada

January, 2005

(C) copyright

2005, Olivier Simard 


$\begin{array}{ll}\begin{array}{l}\text { Library and } \\ \text { Archives Canada }\end{array} & \begin{array}{l}\text { Bibliothèque et } \\ \text { Archives Canada }\end{array} \\ \begin{array}{l}\text { Published Heritage } \\ \text { Branch }\end{array} & \begin{array}{l}\text { Direction du } \\ \text { Patrimoine de l'édition }\end{array} \\ \begin{array}{l}\text { 395 Wellington Street } \\ \text { Ottawa ON K1A ON4 }\end{array} & \begin{array}{l}\text { 395, rue Wellington } \\ \text { Ottawa ON K1A ON4 } \\ \text { Canada }\end{array}\end{array}$

Your file Votre référence ISBN: 0-494-00790-7

Our file Notre référence

ISBN: 0-494-00790-7

NOTICE:

The author has granted a nonexclusive license allowing Library and Archives Canada to reproduce, publish, archive, preserve, conserve, communicate to the public by telecommunication or on the Internet, loan, distribute and sell theses worldwide, for commercial or noncommercial purposes, in microform, paper, electronic and/or any other formats.

The author retains copyright ownership and moral rights in this thesis. Neither the thesis nor substantial extracts from it may be printed or otherwise reproduced without the author's permission.
AVIS:

L'auteur a accordé une licence non exclusive permettant à la Bibliothèque et Archives Canada de reproduire, publier, archiver, sauvegarder, conserver, transmettre au public par télécommunication ou par l'Internet, prêter, distribuer et vendre des thèses partout dans le monde, à des fins commerciales ou autres, sur support microforme, papier, électronique et/ou autres formats.

L'auteur conserve la propriété du droit d'auteur et des droits moraux qui protège cette thèse. $\mathrm{Ni}$ la thèse ni des extraits substantiels de celle-ci ne doivent être imprimés ou autrement reproduits sans son autorisation.
In compliance with the Canadian

Privacy Act some supporting forms may have been removed from this thesis.

While these forms may be included in the document page count, their removal does not represent any loss of content from the thesis.
Conformément à la loi canadienne sur la protection de la vie privée, quelques formulaires secondaires ont été enlevés de cette thèse.

Bien que ces formulaires aient inclus dans la pagination, il n'y aura aucun contenu manquant.

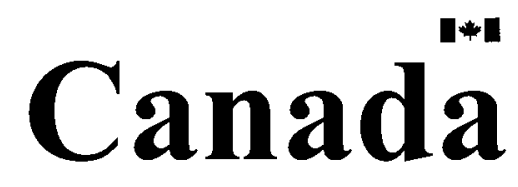




\begin{abstract}
The angular response of the photomultiplier tubes (PMTs) of the Sudbury Neutrino Observatory (SNO) is measured for an extended detector volume. Analytic and Monte Carlo studies of the reflections on the acrylic vessel made possible a full analysis of the optical data taken in a fiducial volume of $550 \mathrm{~cm}$ in radius, a $25 \%$ increase compared to previous analysis methods.

The heavy and light water total extinctions found in that extended volume are consistent with previous measurements. The PMT angular response is also consistent, but extends in the range of incident angle $\left[40^{\circ}, 45^{\circ}\right]$. An approximation of the error due to this new optics on the energy scale uncertainty leads to a reduction of $1.25 \%$ on that uncertainty.
\end{abstract}


Et dire que j'étais venu ici pour faire mes études.

Pour l'instant j'suis rien, mais j'm'en sortirai bien.

Pour ma famille, et ceux qui ont suivi mes démarches.

- Mystik 


\section{Acknowledgements}

Je remercie les membres immédiats et éloignés de mes deux familles. Mon père Jacques et ma mère Céline. Merci de votre constant appui. Vous devez sûrement être très heureux. Mes frères Julien et Alexis. Merci de votre présence et pour les rires. Merci aux Roy à Ottawa, Pierre et Ghislaine, qui ont su m'accueillir comme l'un des leurs. Merci à ma cousine Judith à Montréal avec laquelle j'ai apprécié discuter de la vie, entre deux capitales. Merci aux autres Simard et Gobeil que je ne peux nommer en une page.

Un merci particulier aux membres du devot. Les philosophes Tommy, la morale, et Jean-Philippe, la conscience. L'économe roux Sébastien, toujours aussi tanant. Le casé Patrick, l'homme de la situation. Et les autres: Mathieu, Guillaume, Amélie, Anne-Marie, Marie-Pierre et Yannick. J'en oublie c'est sûr, merci à tous de votre amitié.

This SNO optics study would not have been possible without the help of Ranpal S. Dosanjh. He brought great ideas to Carleton and was a great teacher to me. Thanks for your patience and your wisdom.

Thanks to my collegues at Carleton, namely, Louise Anne Heelan, Étienne Rollin, 
Gordana Tešić and Pierre-Luc Drouin. Thanks to the members of the Carleton optical calibration crew, Ranpal and David Waller. Thanks to the SNO-Carleton faculties: my advisor Alain Bellerive, Richard Hemingway and David Sinclair. Thanks also to Cliff Hargrove for sharing stories. A final thanks to the SNO calibration group for their expertise. Namely José Maneira and Kevin Graham at Queen's for their advise, Peter Skensved, and Bryce Moffat for his milestone optical calibration thesis. 


\section{Statement of Originality}

The work presented in this thesis is the author's own and is original except where noted. All use of work other than the author's and references to additional information are cited in the text.

The author's contribution to the SNO experiment is the optical characterization of the detector. The SNO detector optical model and the original optical calibration methods were determined by Bryce A. Moffat [1]. Periodic optical constant measurements are necessary for SNO to correctly measure neutrino properties; as a member of the SNO calibration group, the author has directly contributed to the SNO results since January 2003.

The first type of light source used for optical calibrations was designed and built by Richard J. Ford [5], and adapted for production data taking by Moffat at Queen's University. Over the years, many light sources have been used. The source used to produce the data analyzed in this thesis was built by Darren R. Grant [7], following the procedures of Moffat, in an attempt to make uniform the source light distribution.

The analysis of the optical data is done through a series of processing steps. The first few steps consist of organizing the raw detector data. This is done through an 
automated program, autosno, that has been developed by many people within the SNO collaboration. The last few data processing steps that lead to the determination of the detector optical constants presented in this thesis were performed by the author. The programs used rely on the QSNO [11] and ROOT [13] libraries which provide supporting routines. The programs were first written by Moffat, and then modified and optimized by Ranpal S. Dosanjh and the author at Carleton University, and by José C. Maneira at Queen's University. A great effort was made to standardize the optical data analysis for updates to the SNO code releases.

The analysis of the AV reflections within the SNO detector geometry was developed by the author. The reflection analytic model developed was tested by the author using the geometrical optics program (QOptics) written by Chris Jillings, Aksel Hallin, and Moffat at Queen's University. The geometry of the SNO detector is contained in SNOMAN $[12,17]$, the SNO simulation and analysis program, developed by many people, mostly based at Oxford University. The validity of the reflection model was checked by the author using the previous existing programs. The simulations presented in this thesis were performed by the author with the help of Dosanjh, who adapted the laser sources simulation command files of Kevin Graham at Queen's to simulate focused beams directed on specific PMTs.

The author's contribution to the official optical data processing was to implement the reflection code in the existing routines to allow the use of optical data in the full SNO fiducial volume. The primary result of this thesis is obtained from the application of the new code on the optical data. It leads to a better parametrization of the PMT angular response as a function of the incident angle of the incoming light.

viii 
The analysis of the uncertainty on the energy response of the SNO detector was done by Moffat using the response processor developed by Mark Boulay. The author's contribution to the new uncertainties found in this thesis was to repeat Moffat's error propagation analysis with new calibration data and analysis methods. 


\section{Contents}

Abstract

Acknowledgements $\quad$ V

Statement of Originality vii

$\begin{array}{ll}\text { Contents } & \mathbf{x}\end{array}$

List of Tables $\quad$ xiv

List of Figures $\quad$ XV

List of Acronyms xix

1 Introduction 1

1.1 Detector Components . . . . . . . . . . . . . . . 2

1.1.1 Acrylic Vessel . . . . . . . . . . . . . . . . . . 2

1.1.2 Photomultiplier Tubes . . . . . . . . . . . . . 3

1.1 .3 Electronics................... . . 5

1.1.4 Light Spectrum . . . . . . . . . . . . . . . 5

1.2 Optical Calibration . . . . . . . . . . . . . . 7

1.2.1 Optical Source Device . . . . . . . . . . . . 7

1.2 .2 Optical Scan . . . . . . . . . . . . . 7

1.2 .3 Optical Data Analysis . . . . . . . . . . . . 9

1.3 Optical Model . . . . . . . . . . . . . . . . . 11

1.3 .1 Parameters ................... 11 


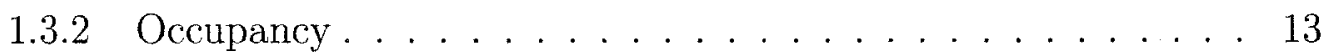

1.4 Occupancy Ratio Method . . . . . . . . . . . . . . . . . . . 14

1.5 PMT Efficiencies Method . . . . . . . . . . . . . . . . . 15

1.6 Synopsis of this Thesis . . . . . . . . . . . . . . 16

2 Optical Data Selection $\quad 18$

2.1 Optical Data Organization . . . . . . . . . . . . . . . . . . 19

2.1.1 Analysis Tools . . . . . . . . . . . . . . . . . . . 19

2.1 .2 PMT Flags . . . . . . . . . . . . . . . . . 20

2.2 Prompt AV Reflections (PAVRs) . . . . . . . . . . . . 23

2.2.1 Default PAVR Selection Criterion . . . . . . . . . . 23

2.2 .2 Light Peaks . . . . . . . . . . . . . . . . . 27

2.3 Motivation for a New PAVR Cut . . . . . . . . . . . . . . 29

3 An Analytic Model for AV Reflections 33

3.1 Return to the Basics ... . . . . . . . . . . . . . 33

3.2 Analytic Model . . . . . . . . . . . . . . . . 36

3.2.1 Model Geometry . . . . . . . . . . . . . . . . . . 36

3.2 .2 Derivations . . . . . . . . . . . . . . . . 38

3.2 .3 Solutions . . . . . . . . . . . . . . . . 40

3.2 .4 Probabilities. . . . . . . . . . . . . . 43

3.3 Merging the Analytic Model with the Existing Optics . . . . . . . . . 45

3.3.1 Transmitted Light Information . . . . . . . . . . . . . . . 46

3.3 .2 Reflected Light Information . . . . . . . . . . . . . . . . 47

3.4 Summary . . . . . . . . . . . . . . . . . . . 52

4 Monte Carlo Checks $\quad \mathbf{5 5}$

4.1 Introduction . . . . . . . . . . . . . . . . . 55

4.1 .1 Time Information . . . . . . . . . . . . . . 56

4.1.2 Light Production . . . . . . . . . . . . . . 58

4.2 Laserdisk Source . . . . . . . . . . . . . . . . . 59

4.2 .1 Laserdisk Time Information . . . . . . . . . . . . . 60

4.3 Photon Bomb Source . . . . . . . . . . . . . . . . . . . . . . . 62 
4.3.1 Photon Bomb Time Information . . . . . . . . . . . . . 63

4.4 Combined Timing Information . . . . . . . . . . . . . . . 65

4.5 Comparison with the Analytic Model . . . . . . . . . . . . . 67

4.5 .1 Source Configurations . . . . . . . . . . . . 68

4.5 .2 Results . . . . . . . . . . . . . . . 70

4.6 Discussion . . . . . . . . . . . . . . . . . 71

4.6 .1 Time Differences . . . . . . . . . . . . . . . 72

4.6.2 Reflection Causes and Types . . . . . . . . . . . 76

4.6 .3 Summary . . . . . . . . . . . . . . . . 76

$\begin{array}{lll}5 & \text { Implementation of the New Code } & 77\end{array}$

5.1 Adding the New Code . . . . . . . . . . . . . . . . 77

5.1 .1 Calculations . . . . . . . . . . . . . 78

5.1 .2 Speed or Precision . . . . . . . . . . . . . . 78

5.2 Class Parameters . . . . . . . . . . . . . . . . . . . . . 79

5.2 .1 Source Position Radius . . . . . . . . . . . . . . . . 79

5.2 .2 Time Window Acceptance . . . . . . . . . . . . . . . . . 79

5.2 .3 Summary . . . . . . . . . . . . . . . . . 82

6 Results of the Enhanced Processing Method 84

6.1 Changes in the Statistics . . . . . . . . . . . . . . 85

6.1.1 Statistics of High Radius Runs . . . . . . . . . . . . 86

6.1 .2 Statistics of an Entire Scan . . . . . . . . . . . . 88

6.2 Fit Results . . . . . . . . . . . . . . . . . . . . . . . 91

6.2.1 Uncertainties . . . . . . . . . . . . . . 93

6.2.2 Media Attenuations . . . . . . . . . . . . . . . . . . 94

6.2.3 PMT Angular Response . . . . . . . . . . . . . . . 96

6.2.4 Quality of the Fits . . . . . . . . . . . . . . 99

6.3 Uncertainties due to the Optics . . . . . . . . . . . . . . . . 100

6.3.1 The Detector Response Function . . . . . . . . . . . 100

6.3 .2 Uncertainty on the Energy Scale . . . . . . . . . . . 101

xii 
7 Discussion and Conclusion $\quad 103$

7.1 More on the Enhanced Method . . . . . . . . . . . . . . 103

7.1.1 Time Windows . . . . . . . . . . . . . . . . . . . . . 104

7.1 .2 Uncertainties . . . . . . . . . . . . . . . . . . 104

7.2 Comparison of the Optical Constants . . . . . . . . . . . . . . 105

7.2 .1 Media Attenuations . . . . . . . . . . . . . . . . 105

7.2 .2 PMT Response . . . . . . . . . . . . . . . 106

7.2 .3 Uncertainties . . . . . . . . . . . . . . . 107

7.3 Improvements Needed for the NCD Phase . . . . . . . . . . . . . . 107

7.3 .1 Fit Alternatives . . . . . . . . . . . . . . 107

7.4 Conclusion . . . . . . . . . . . . . . . . . . 109

$\begin{array}{ll}\text { A Laserdisk Command File } & 111\end{array}$

B MC Time Distributions $\quad 113$

B.1 Laserdisk Source Radius . . . . . . . . . . . . . . . . . . . . . . . 113

B.2 Time Distribution Fits . . . . . . . . . . . . . . . . . . 117

B.3 Comments on SNOMAN . . . . . . . . . . . . . . . . . . . 117

B.3.1 Laserball Source . . . . . . . . . . . . . . . . . 117

B.3.2 3D-PMT Model . . . . . . . . . . . . . . . . . 119

C Additional Fit Results $\quad \mathbf{1 2 5}$

C.1 Comparisons with Contaminated Data Fits . . . . . . . . . . . . . . 125

C.1.1 Media Attenuations . . . . . . . . . . . . . . . . . 126

C.1.2 PMT Response . . . . . . . . . . . . . . . . . . . . 129

C.1.3 Summary . . . . . . . . . . . . . . . . . 130

$\begin{array}{lr}\text { Bibliography } & 138\end{array}$

xiii 


\section{List of Tables}

2.1 PMT flags used in the optical data selection process. . . . . . . . 20

3.1 Example of AV reflection solutions predicted by the analytic model. . 42

3.2 Analytic model AV reflection solution types. . . . . . . . . . . . 43

3.3 Reflected light information as a function of the source position radius for sources on the negative $z$-axis. . . . . . . . . . . . . . . 49

4.1 Monte Carlo sources settings. . . . . . . . . . . . . . . . . . . . 69

4.2 Laserdisk source configuration parameters and data filters. . . . . . . 70

4.3 Time differences comparison for selected PMTs of configuration A. . . 73

4.4 Time differences comparison for selected PMTs of configuration B. . . 73

4.5 Time differences comparison for selected PMTs of configuration C. . . 74

4.6 Time difference estimators comparison for PMTs of each configuration. 75

6.1 QOptics flag details. . . . . . . . . . . . . . . 86

6.2 Processing methods for the October 2003 scan. . . . . . . . . . . . 93

6.3 Systematic uncertainties. . . . . . . . . . . . . . . . . . 94

6.4 Energy scale uncertainties due to the optics for different processing methods and fiducial volumes. . . . . . . . . . . . . . . . . 102

B.1 Gaussian fit parameters and time differences between reflection and prompt light peaks for different laserdisk source radius sizes. . . . . . 114

C.1 More processing methods for the October 2003 scan. . . . . . . . . 126

C.2 Summary of the optical parameters variations with respect to the processing method used. . . . . . . . . . . . . . . 137

xiv 


\section{List of Figures}

1.1 Acrylic vessel and its support structure. . . . . . . . . . . 3

1.2 Typical SNO PMT and its reflector assembly. . . . . . . . . 4

1.3 Cerenkov spectrum convolved with the relative PMT quantum effi-

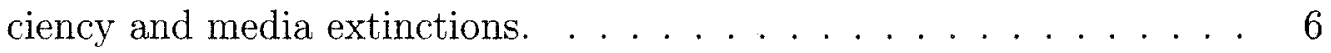

1.4 Laserball assembly. . . . . . . . . . . . . . . . . . . . . . 8

1.5 Source manipulator system and allowed positioning regions. . . . . 10

2.1 View of the PMTs flagged for a source at the center of the detector. . 22

2.2 Maximum incident angle of incoming light on PMT surfaces as a function of the source position radius. . . . . . . . . . . . 24

2.3 Vector representation of the source and the PMT positions within the detector. . . . . . . . . . . . . . . . . . 25

2.4 View of the PMTs flagged for a source at high radius. . . . . . . 26

2.5 Example of a time distribution plot for a central run. . . . . . . . 28

2.6 Time distribution plot for runs of the October 2003 scan. . . . . . 28

3.1 Fresnel reflection coefficient applied to SNO refraction indices as a function of the incident angle on the AV . . . . . . . . . 35

3.2 Vector geometry and coordinate systems used in the AV reflection analytic model. . . . . . . . . . . . . . . . . . . 37

3.3 Analytic functions of the incident and reflection angles on the AV surface. . . . . . . . . . . . . . . . . 4 41

3.4 Reflection predictions of the analytic model for PMTs located at the equator and at the top of the detector. . . . . . . . . . 44 
3.5 Scatter plot of the incident angle on the PMT surface versus the prompt light path as a function of the source position radius. . . . . 46

3.6 Incident angle on the PMT surface distributions for sources along the negative $z$-axis. . . . . . . . . . . . . . . . . . 48

3.7 Angle of incidence on the inner AV surface as a function of the source position radius. . . . . . . . . . . . . . 50

3.8 Scatter plot of the incident angle on the PMT surface versus the reflection light path as a function of the source position radius. . . . . 51

3.9 Scatter plot of the time differences between the reflected and prompt light paths versus the PMT positions as a function of the source position radius. . . . . . . . . . . . . . . . . 53

3.10 Scatter plot of the time differences between the reflected and prompt light paths versus the PMT positions as a function of the source position radius for a maximum of two reflections. . . . . . . . 54

4.1 Monte Carlo laserdisk source and various light paths. . . . . . . . . 60

4.2 View of the PMT hits obtained with a Monte Carlo laserdisk source. 61

4.3 Typical time distribution obtained using the laserdisk source. . . . . . 62

4.4 View of the PMT hits obtained with a Monte Carlo photon bomb source. . . . . . . . . . . . . . . . . 63

4.5 Typical time distribution obtained using the photon bomb source. . . 64

4.6 Monte Carlo sources complementary information. . . . . . . . . . . 65

4.7 View of the PMT hits obtained in various laserdisk configurations. . . 71

4.8 View of the PMT filtered hits obtained in laserdisk configurations. . . 72

5.1 Time windows acting on the time data of a single PMT channel. . . . 82

5.2 Simplistic view of the modified cut due to prompt AV reflected light. 83

6.1 Effect of applying the new PAVR cut on the statistics of run 34865. . 87

6.2 Effect of applying the new PAVR cut on the statistics of run 34964. . 87

6.3 Effect of applying the new PAVR cut on the statistics of the runs at $420 \mathrm{~nm}$ from the October 2003 scan. . . . . . . . . . . 90 
6.4 Net difference in statistics between the enhanced and the standard processing methods for runs at $420 \mathrm{~nm}$ from the October 2003 scan. . 92

6.5 $\mathrm{D}_{2} \mathrm{O}$ attenuation as a function of wavelength for the October 2003 scan. 95

$6.6 \mathrm{H}_{2} \mathrm{O}$ attenuation as a function of wavelength for the October 2003 scan. 95

6.7 PMT angular response at $500 \mathrm{~nm}$ as a function of incident angle for the October 2003 scan. . . . . . . . . . . . . . . . . . . . 97

6.8 Statistical and systematic errors of the PMT angular response at 500 $\mathrm{nm}$ for the October 2003 scan. . . . . . . . . . . . . . . . . 97

6.9 Reduced $\chi^{2}$ obtained from the optical data fits. . . . . . . . . . . 99

B.1 Monte Carlo laserdisk source PMT time distributions and fits to double Gaussian functions. . . . . . . . . . . . . . . . . . 115

B.2 Monte Carlo laserdisk source PMT time distributions for different radius sizes. . . . . . . . . . . . . . . . . 116

B.3 Monte Carlo PMT time distributions for various laserdisk source configurations used for the comparisons to the analytic model. . . . . . 118

B.4 Monte Carlo laserdisk source prompt and reflected light peak fits. . . 121

B.5 Monte Carlo PMT time distributions for various photon bomb configurations. . . . . . . . . . . . . . . . . . . . . 122

B.6 Monte Carlo PMT time distributions for various laserdisk configurations obtained from selected channels. . . . . . . . . . . . . . 123

B.7 Monte Carlo PMT time distributions for various photon bomb configurations obtained from a group of selected channels. . . . . . . . 124

C.1 $\mathrm{D}_{2} \mathrm{O}$ and $\mathrm{H}_{2} \mathrm{O}$ attenuations obtained from runs with $R_{S}^{\max }=500$ and

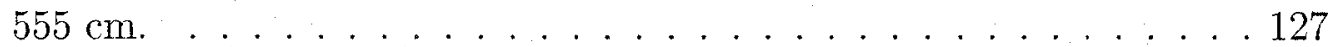

C.2 Effect of adding high radius runs on the $\mathrm{D}_{2} \mathrm{O}$ and $\mathrm{H}_{2} \mathrm{O}$ attenuations when using various processing methods. . . . . . . . . . . . . . . 128

C.3 Relative PMT angular response obtained from runs with $R_{S}^{\max }=500$ cm. . . . . . . . . . . . . . . . . . 131

C.4 Relative PMT angular response obtained from runs with $R_{S}^{\max }=555$ cm. . . . . . . . . . . . . . . . . . . . 132 
C.5 Effect of adding high radius runs on the relative PMT angular response when using the standard processing method. . . . . . . . 133

C.6 Effect of adding high radius runs on the relative PMT angular response when no cut is applied for AV reflections. . . . . . . . . 134

C.7 Effect of adding high radius runs on the relative PMT angular response when using the enhanced processing method. . . . . . . . 135

C.8 Net effect of adding high radius runs and using the enhanced processing method on the relative PMT angular response. . . . . . . 136 


\section{List of Acronyms}

\begin{tabular}{|c|c|}
\hline AV & Acrylic Vessel \\
\hline CHCS & CHannel Calibration Status \\
\hline DQXX & Data acQuisition bit \\
\hline LB & LaserBall \\
\hline $\mathrm{MC}$ & Monte Carlo \\
\hline MPE & Multiple Photo-Electron \\
\hline NCD & Neutral Current Detector \\
\hline $\mathrm{NDF}$ & Number of Degrees of Freedom \\
\hline NHIT & Number of PMTs signalling a hit in an event \\
\hline OCA & Optical CAlibration \\
\hline PAVR & Prompt AV Reflection \\
\hline PCA & Pmt CAlibration \\
\hline PMT & PhotoMultiplier Tube \\
\hline PMTR & PhotoMultiplier Tube angular Response \\
\hline PSUP & Pmt SUPport structure \\
\hline QOCA & SNO Optical CAlibration module in QSNO \\
\hline QSNO & SNO analysis package integrated with ROOT \\
\hline $\mathrm{RCH}$ & Root Calibration Histogram \\
\hline RMS & Root Mean Square \\
\hline RSP & SNO detector Response Processor \\
\hline ROOT & Object-oriented data analysis package in $\mathrm{C}++$ \\
\hline SNO & Sudbury Neutrino Observatory \\
\hline SNOMAN & SNO Monte carlo and ANalysis program \\
\hline UV & UltraViolet \\
\hline XSNOED & X-windows SNO Event Display \\
\hline
\end{tabular}




\section{Chapter 1}

\section{Introduction}

The Sudbury Neutrino Observatory (SNO) is a water Cerenkov detector located two kilometers underground in the Creighton mine near Sudbury, Ontario, Canada. It was designed to measure the solar neutrino flux and to solve the solar neutrino problem. The detector consists of 1000 tonnes of heavy water $\left(\mathrm{D}_{2} \mathrm{O}\right)$ held in an acrylic vessel (AV) which is shielded by light water $\left(\mathrm{H}_{2} \mathrm{O}\right)$ in the cavern. SNO uses 9438 photomultiplier tubes (PMTs) that look inward at the heavy water volume for Čerenkov light being emitted by relativistic charged particles.

The energy of a neutrino is a function of the total Cerenkov radiation emitted when it interacts with a deuterium (D) atom. To precisely measure this energy, the detector components need to be calibrated in many different ways. One such way is the optical calibration of the detector [1], which provides a measurement of the PMT array response and the media attenuations, that are then used to convert the signal into neutrino energy, position, and direction.

This introduction is a short description of the detector as an optical device for 
the first phases of the SNO experiment: the pure $\mathrm{D}_{2} \mathrm{O}$ phase (December 1999 to May 2001) and the salt phase (May 2001 to August 2003). Detailed explanations of the optical calibration process and the basics of the optical data analysis during those phases can be found in [1]. A description of the SNO detector can be found in $[2]$.

\subsection{Detector Components}

In the SNO detector, the Cerenkov photons can be absorbed, re-emitted, attenuated, and refracted before being detected by the PMT array. Understanding the geometry of the detector and the interactions of light propagating is essential in extracting the correct optical response.

\subsubsection{Acrylic Vessel}

The $\mathrm{D}_{2} \mathrm{O}$ is contained in an acrylic vessel which has been optically characterized. This AV is a $12 \mathrm{~m}$ diameter hollow sphere built from 125 panels with a nominal thickness of $5.5 \mathrm{~cm}$. The equatorial band of the AV has thicker panels $(11.4 \mathrm{~cm})$ to accommodate support ropes. The top of the AV has a chimney, also called the neck, which is $8 \mathrm{~m}$ high and $1.5 \mathrm{~m}$ in diameter. It serves as the opening to deploy calibration sources into the $\mathrm{D}_{2} \mathrm{O}$ volume. The AV sphere is UV light transparent but not the chimney. Figure 1.1 shows the AV panels, the support ropes, and the chimney. 


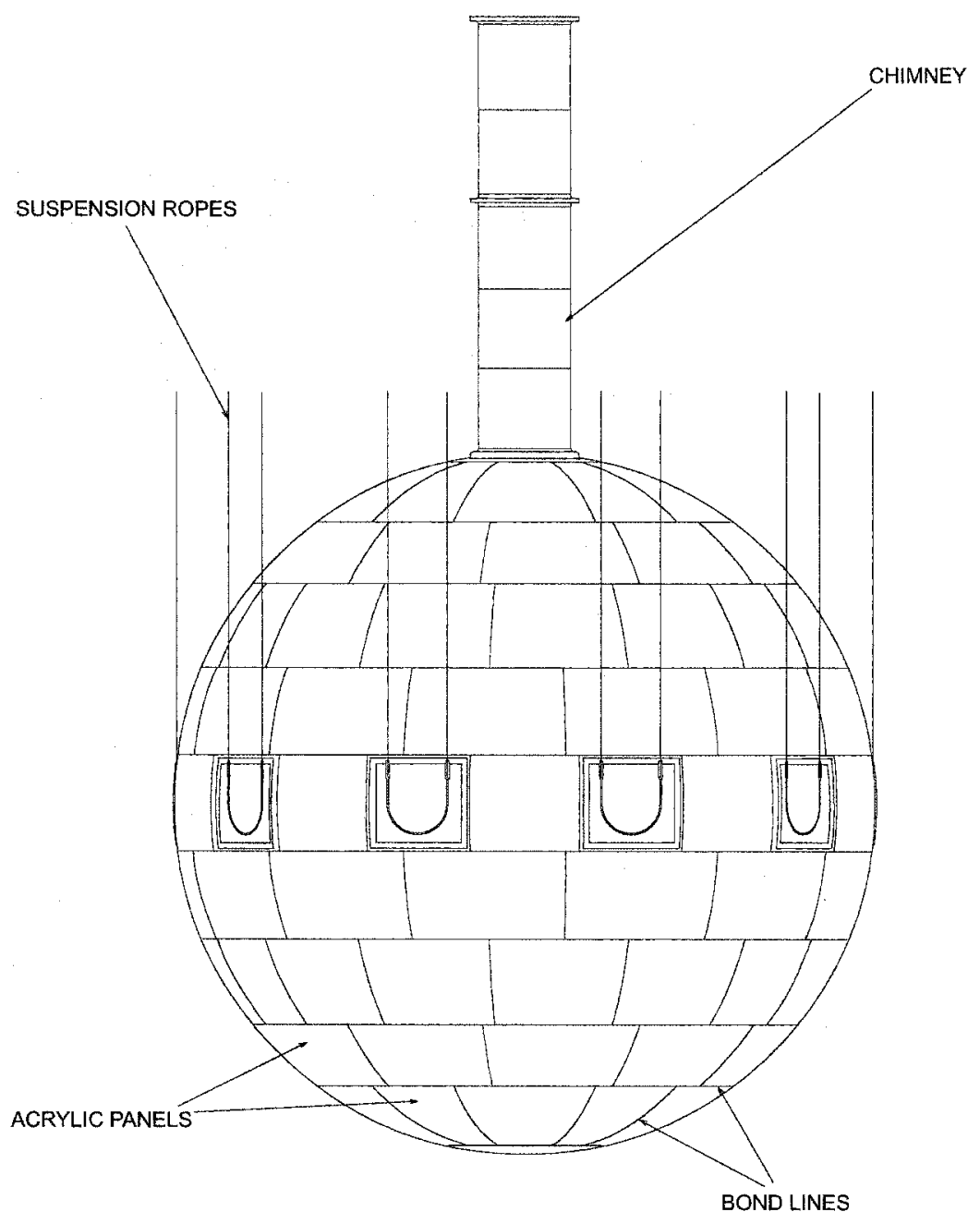

Figure 1.1: The acrylic vessel and its support structure. The belly plates are the equatorial thicker acrylic panels where the suspension ropes are anchored.

\subsubsection{Photomultiplier Tubes}

A photomultiplier tube is a device used to detect photons. SNO uses a total of 9829 R1408 $20 \mathrm{~cm}$ PMTs manufactured by Hamamatsu. They were selected to provide the best compromise between high photocathode coverage, good single photon detection efficiency, small transit time spread (1.5 ns), low noise rate, good reliability and, of 


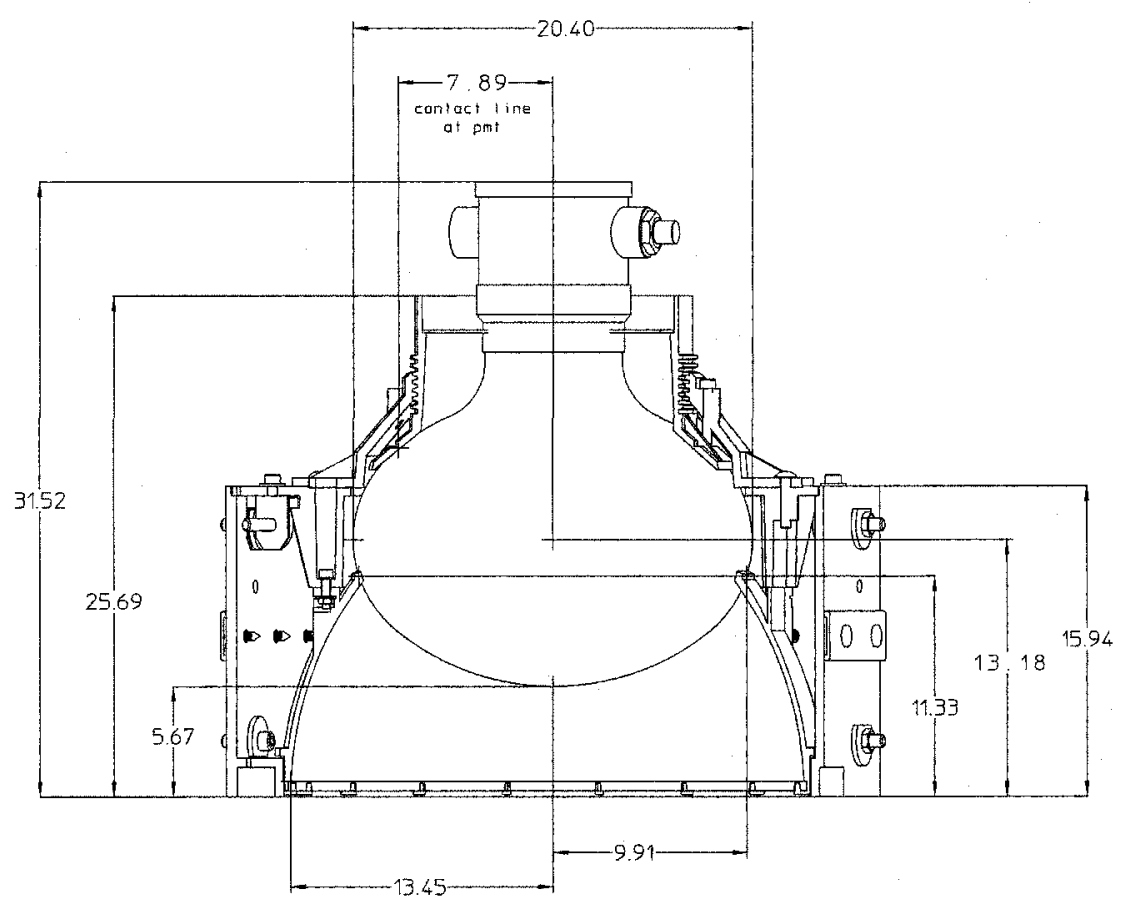

Figure 1.2: A typical SNO PMT and its reflector assembly. Note that dimensions are given in $\mathrm{cm}$.

course, low cost.

The PMTs are positioned on the phototube support structure (PSUP) in the $\mathrm{H}_{2} \mathrm{O}$ surrounding the AV. The PSUP consists of a $17 \mathrm{~m}$ diameter stainless steel geodesic sphere. The inward looking PMTs point to the center of the detector; their surface normal vectors almost point to the origin of the PSUP. The photocathode coverage of the $\mathrm{D}_{2} \mathrm{O}$ volume is about $34 \%$. To increase the surface covered without increasing the number of PMTs of the array, $27 \mathrm{~cm}$ diameter reflectors, or concentrators, were added to the PMTs to force the photons to reflect and hit the photocathode. The effective coverage of the volume is about $59 \%$ with the added PMT reflectors, but reduces to $54 \%$ when the reflectivity of the concentrators is considered. Figure 1.2 shows a typical PMT with reflectors and its dimensions. 


\subsubsection{Electronics}

The electronic system of SNO was designed to measure signals coming from single photons. All the PMT electronic channels are linked to the data acquisition system (DAQ) that is located above the water volume. After light has been captured by the PMTs, the electronic system combines the channel pulses into $400 \mathrm{~ns}$ long events. The system is triggered if many PMT channels fire within a window of $100 \mathrm{~ns}$. The events are stored whenever the detector trigger conditions are met. The number of PMTs that fire pulses in an event is called NHIT. The events are basically made of two observables: charge and relative timing. The charge is an integrated sum of the triggered PMT channel pulses. The relative timing is used to group the channels and to recognize the pattern of an event.

\subsubsection{Light Spectrum}

A characterization of all the detector components is necessary to understand the spectrum of the light to be detected. The Čerenkov spectrum $[3,4]$ is convolved with the transmission of the SNO media and the PMT photon detection efficiency to obtain the light spectrum to be observed by SNO as depicted in Figure 1.3. The AV dominates the absorption below $300 \mathrm{~nm}$. The relative PMT quantum efficiency (QE) has a maximum of $21.5 \%$ at $440 \mathrm{~nm}$ [1] and dominates the absorption above 700 $\mathrm{nm}$. The spectrum of the photons in SNO is contained in the interval $300 \leqslant \lambda \leqslant 650$ $\mathrm{nm}$, which defines the wavelengths to be used in optical calibrations. 

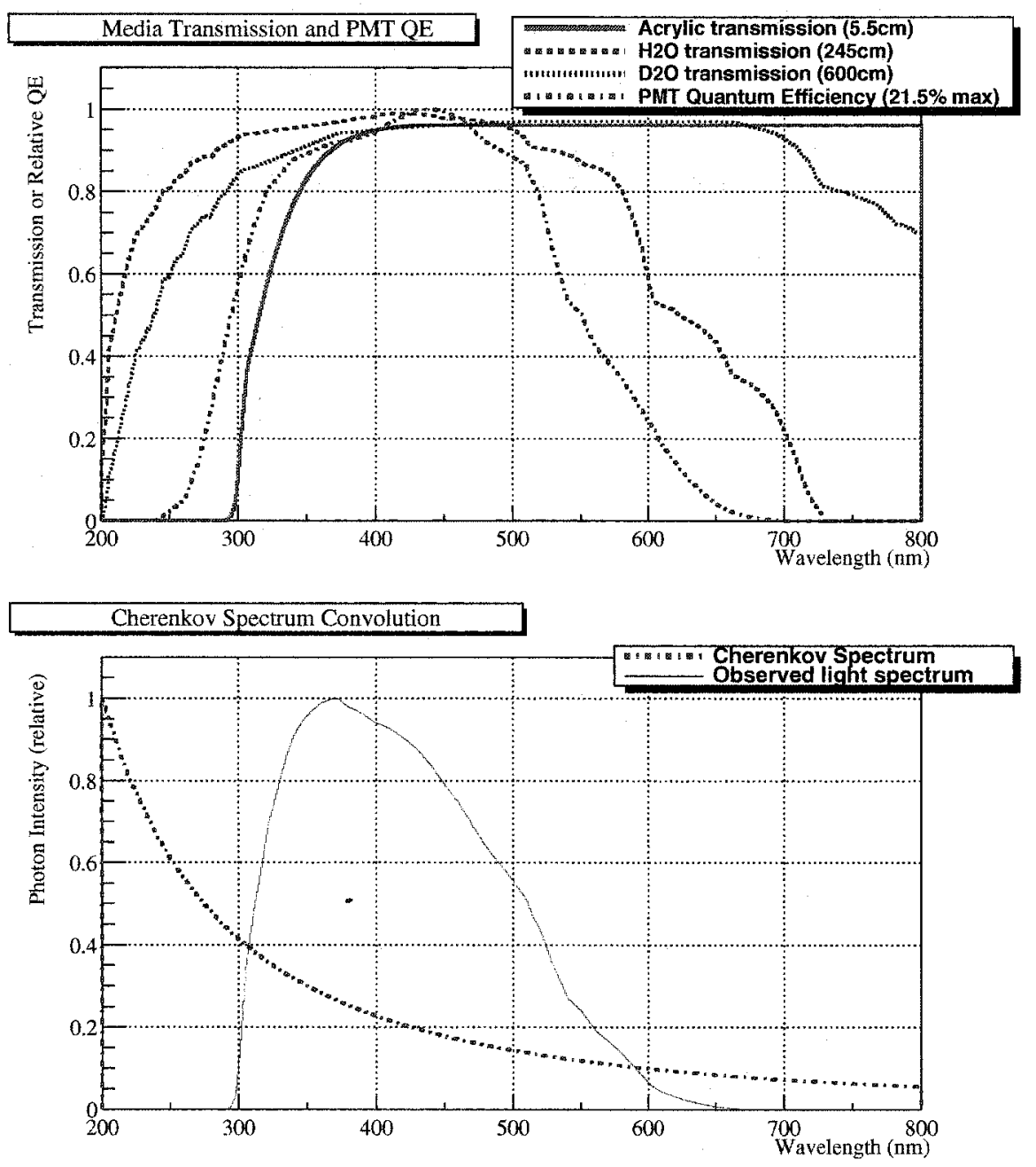

Figure 1.3: Čerenkov spectrum convolved with the relative PMT quantum efficiency and media extinctions. The detector is only sensitive to light between 300 and 650 nm. Figure courtesy of Dr. Bryce Moffat [1]. 


\subsection{Optical Calibration}

The following is a summary of the optical calibration process during the pure $\mathrm{D}_{2} \mathrm{O}$ and salt phases of SNO.

\subsubsection{Optical Source Device}

The SNO calibration group uses multiple sources that serve different calibration purposes. Optical calibrations are performed with a Nitrogen $\left(\mathrm{N}_{2}\right)$ laser system $[5,6]$ connected to a source deployed in the detector called the laserball $[1,7]$. The laser system has a natural wavelength of $337 \mathrm{~nm}$ and emits about $1.7 \times 10^{14}$ photons per pulse. Dye cells are used to change that wavelength such that six wavelengths are available for the calibrations: $\lambda=337,365,386,421,500$, and $620 \mathrm{~nm}$. The laserball shown in Figure 1.4 has a diameter of $10.9 \mathrm{~cm}$. It is made of a diffuse material that outputs the nearly monochromatic laser light almost isotropically into the detector.

\subsubsection{Optical Scan}

The optical calibration is done in many runs as the detector response is sensitive to the source position and to the wavelength of the light. An optical scan consists of $N_{\text {run }}$ runs taken at different positions and wavelengths. A source manipulator is used to position the laserball in the detector. The system uses three ropes attached to a pivot on top of the source assembly to move the source in the volume contained by the AV. The positions can either be in the $x z$ or the $y z$ planes of the detector, where $(x, y, z)=(0,0,0)$ defines the center of the detector. Otherwise the source can also be deployed with only one rope in guide tubes from the top to the bottom of 


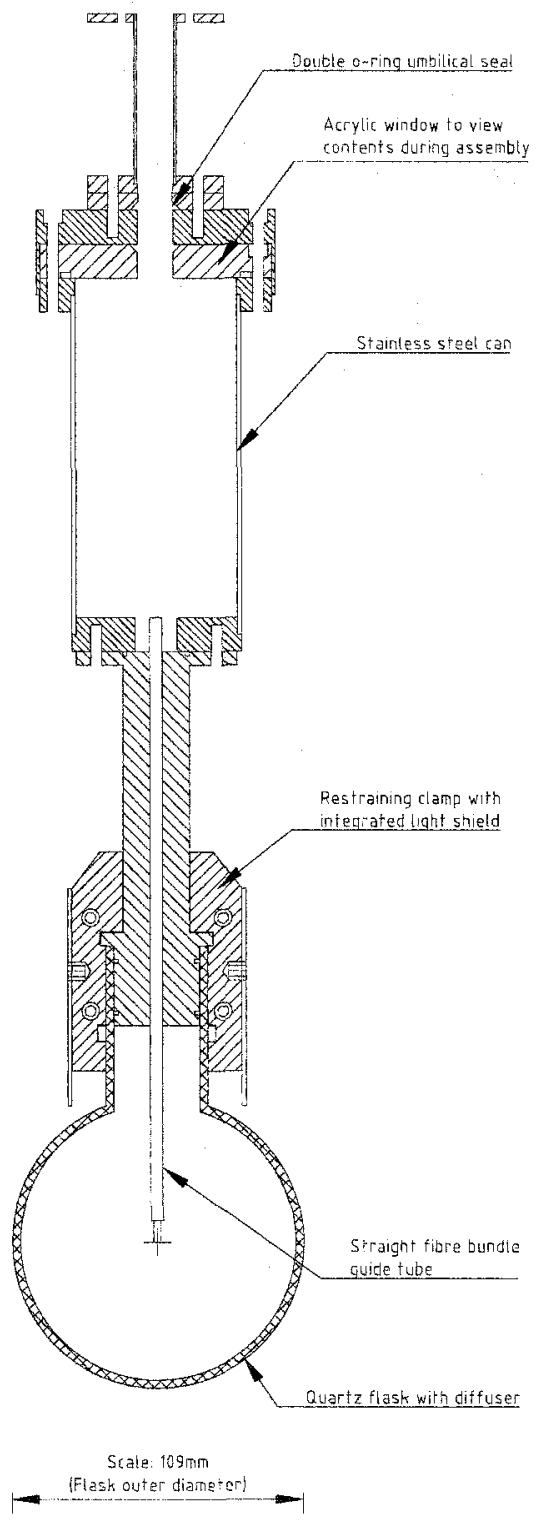

Figure 1.4: The laserball assembly. 
the detector. In this case the position can only change along an axis parallel to the $z$-axis of the detector. Figure 1.5 shows an example view of the manipulator system ropes. The dots drawn on the grid represent source positions taken during a typical scan.

Each run contains $N_{P M T}$ valid PMTs, or channels, that are in a good state to acquire data. Central runs, taken at the center of the detector, are important since they provide information about the symmetry of the spherical detector; they are also used as normalization runs for the analysis of the optical data in the pure $\mathrm{D}_{2} \mathrm{O}$ and salt phases of the experiment. A typical central run lasts between 30 minutes and two hours with a laser pulse emission rate of approximately $40 \mathrm{~Hz}$. A typical off-center run lasts 15 minutes, also with a laser pulse emission rate of $40 \mathrm{~Hz}$. The latter are required to measure the response of the detector in different regions of the water volume. Equivalently, these runs are used to measure the response of the PMTs to the incoming light at different incident angles, and to measure the media attenuations by comparing the light path length differences with the central run.

\subsubsection{Optical Data Analysis}

The analysis of the optical data relies on the optical model of the detector presented in Section 1.3. The light used for that analysis is called prompt light, which is the direct light from the source to a given PMT. Once the position and wavelength are known for a specific run, the SNO optical calibration software performs a series of calculations. For instance, the incident angles on the PMTs and the distances in the different media from the source to each PMT are calculated from the detector geometry. It also analyzes the prompt light charge and time data recorded for each 


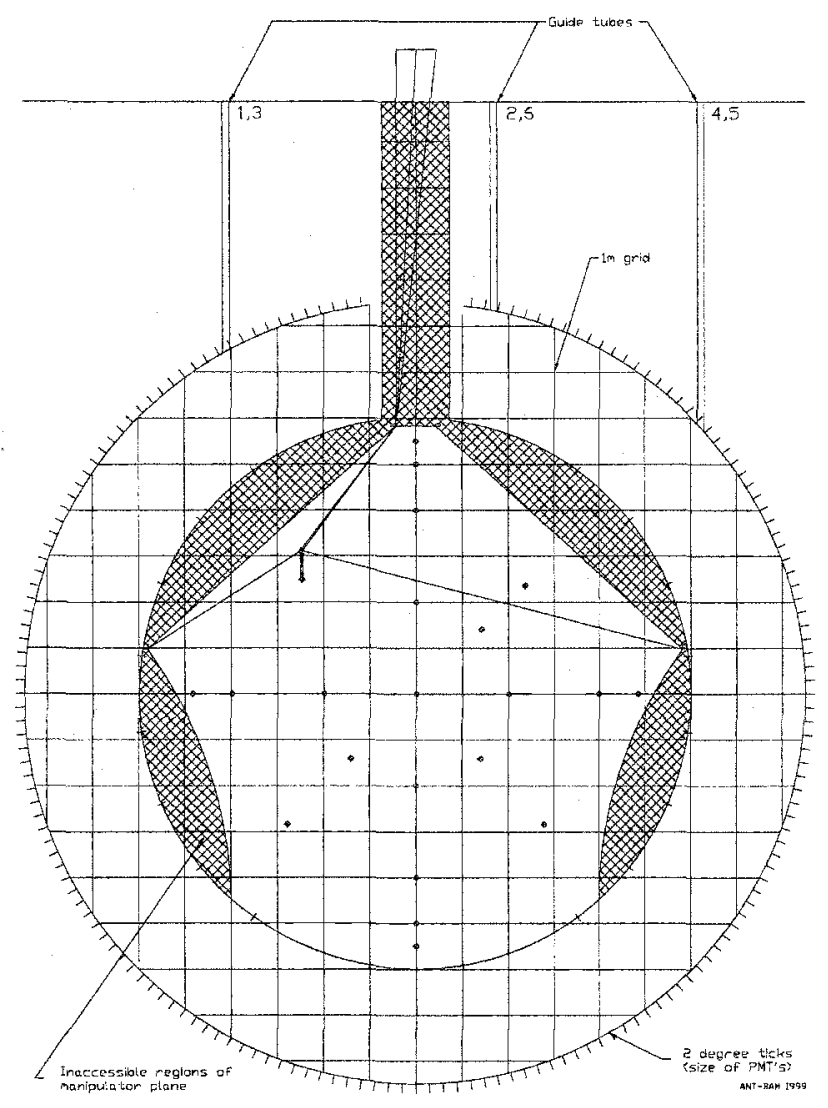

Figure 1.5: Source manipulator system and allowed positioning regions. The source is moved using three ropes in one plane of the detector. The source positions of a typical scan are represented by the dots on the grid. The shadowed regions are unaccessible to the manipulator system. The guides tubes allow deployment of the source in the volume outside the AV. Figure courtesy of Dr. Bryce Moffat [1].

PMT channel. In this way, each PMT is considered as a data point. A fit of the optical model to the data provides the detector optical constants, that are used in the neutrino data analysis to determine the energy, position, and direction associated with neutrino events. 


\subsection{Optical Model}

The optical model incorporates the detector components previously mentioned, plus the details of light collection in a typical phototube. Most of the light detected in a run is contained in what is called the prompt peak. The light selected for the optical data analysis has a half width of 4 ns centered on the prompt peak. It is a smaller time window than the 10 ns window used in the neutrino energy estimation analysis.

The detector optical response is characterized by the optical constants. The latter are parameters of the model extracted from the fit of the model to the data. The PMT response as a function of the light angle of incidence, or the PMT angular response (PMTR), and the media attenuation coefficients, or inverse attenuation lengths, are the most important optical constants to measure.

\subsubsection{Parameters}

The prompt intensity, $N_{i j}$, observed for run $i$ in PMT $j$ has been defined first in [1] and then modified in [8] and is parametrized as follows:

$$
N_{i j}^{\text {model }}=N_{i} \Omega_{i j} R_{i j} L_{i j} T_{i j} \epsilon_{i j} e^{-\left(d_{\mathrm{d}} \alpha_{\mathrm{d}}+d_{\mathrm{a}} \alpha_{\mathrm{a}}+d_{\mathrm{h}} \alpha_{\mathrm{h}}\right)},
$$

where the multiplicative factors can be either calculated or extracted from the laserball data: 
$N_{i} \quad$ The number of photons emitted by the laserball in run $i$ and detected within the prompt timing window at each PMT. This term is the intensity normalization for this run and cannot be measured directly with great precision.

$\Omega_{i j} \quad$ The solid angle in run $i$ for the PMT $j$, i.e. the acceptance. This term is calculated based on the detector geometry.

$R_{i j} \quad$ Additional geometric correction factors which describe the phototube and reflector angular response beyond the solid angle (also referred as PMTR). They correspond to the light transmission of the phototube glass material and the reflectivity of the reflectors. It is extracted from the laserball data.

$L_{i j} \quad$ Laserball light distribution expressed as a function of angles relative to the laserball position. $L_{i j} \equiv L_{i j}\left(\theta_{L B}, \phi_{L B}\right)$. It is extracted from the laserball data.

$T_{i j} \quad$ Fresnel transmission coefficients for the $\mathrm{D}_{2} \mathrm{O} /$ acrylic $/ \mathrm{H}_{2} \mathrm{O}$ interfaces. These terms are calculated analytically, given a source and a PMT position.

$\epsilon_{i j} \quad$ The relative quantum efficiency in run $i$ for the PMT $j$. It is used to characterize the wavelength-dependent probability of registering a hit. In principle, it can be extracted from the data.

$d_{\mathrm{d}, \mathrm{a}, \mathrm{h}} \quad$ Light path lengths through the heavy water (d), acrylic (a) and light water (h), respectively. These distances are calculated based on the detector geometry. 
$\alpha_{\mathrm{d}, \mathrm{a}, \mathrm{h}} \quad$ Attenuation coefficients for the heavy water (d), acrylic (a) and light water (h), respectively. These attenuations are extracted from the laserball data.

\subsubsection{Occupancy}

In a run $i, N_{i}^{\text {pulses }}$ laser pulses are expected to be detected by the PMTs. In a PMT $j$, the raw occupancy is defined as the ratio of the prompt pulses detected to the total pulses emitted by the laser:

$$
O_{i j}^{\text {raw }}=\frac{N_{i j}^{\text {prompt }}}{N_{i}^{\text {pulses }}}
$$

This number is then corrected for multiple photons, coming from a single laser pulse, reaching a PMT. When that happens, the PMT registers only one hit, but many photoelectrons are produced in the tube. The correction assumes Poisson statistics for multiple photoelectrons (MPE) being produced in the PMT [9]. The MPE-corrected prompt occupancy is

$$
O_{i j}^{\text {prompt }}=-\ln \left(1-O_{i j}^{\text {raw }}\right)
$$

The number of prompt photons detected by a PMT $j$ can then be found by multiplying this ratio by the normalization, which defines the estimator for the prompt intensity:

$$
N_{i j}^{\text {data }} \equiv N_{i j}^{\mathrm{MPE}, \text { prompt }}=N_{i}^{\text {pulses }} O_{i j}^{\mathrm{prompt}}
$$

Sections 1.4 and 1.5 summarize how the data is organized before the fit is performed. 


\subsection{Occupancy Ratio Method}

The model of Equation (1.1) has thousands of parameters for an entire scan. The principle of the occupancy ratio $(O R)$ method is to compare two different run occupancies. One run acts as the normalization (subscript $i=0$ ), and the other is the run under study. For both the $\mathrm{D}_{2} \mathrm{O}$ and salt phases, the normalization run used is usually a central run, taking advantage of the detector symmetry. The occupancy ratio is calculated for each PMT such that the PMT efficiencies $\epsilon_{i j}$ cancel, assuming the efficiencies do not vary during a scan. This approach reduces considerably the number of parameters allowed to vary in the fit and gives a number of degrees of freedom of typically 150,000 , depending on the number of runs in a scan.

The solid angles $\Omega_{i j}$ and the transmission coefficients $T_{i j}$ terms of Equation (1.1) can be directly calculated from the geometry of the detector given the source position in run $i$ and the positions of all the $j$ PMTs. To reduce even more the number of varying parameters, the data is corrected with the factor

$$
C_{i j}=\frac{\Omega_{0 j} T_{0 j}}{\Omega_{i j} T_{i j}}
$$

so that the data occupancy ratio is defined as

$$
O R_{i j}^{\text {data }}=\frac{N_{i j}^{\text {data }}}{N_{0 j}^{\text {data }}} \times C_{i j}=\frac{N_{i j}^{\text {data }}}{N_{0 j}^{\text {data }}}\left(\frac{\Omega_{0 j} T_{0 j}}{\Omega_{i j} T_{i j}}\right)
$$

The model occupancy ratio is then given by:

$$
O R_{i j}^{\text {model }}=\frac{N_{i j}^{\text {model }}}{N_{0 j}^{\text {model }}} \times C_{i j}=\frac{N_{i}}{N_{0}}\left(\frac{R_{i j} L_{i j}}{R_{0 j} L_{0 j}}\right) e^{-\left(\delta d_{\mathrm{d}} \alpha_{\mathrm{d}}+\delta d_{\mathrm{a}} \alpha_{\mathrm{a}}+\delta d_{\mathrm{h}} \alpha_{\mathrm{h}}\right)},
$$


where $\delta d=d_{i j}-d_{0 j}$ is the path difference in one of the three media. The distance $d_{i j}$ is calculated from the off-center run source position to the PMT $j$; the distance $d_{0 j}$ is calculated from the central run source position to the same PMT $j$. The only parameters left to be fitted are the run normalizations $N_{i}$, the PMT response $R_{i j}$, the laserball distribution $L_{i j}$, and the media attenuation coefficients $\alpha_{\mathrm{d}, \mathrm{a}, \mathrm{h}}$. The fit for these optical parameters is done by minimizing the chi-square differences between the data and the model:

$$
\chi^{2}=\sum_{i}^{N_{\text {run }}} \sum_{j}^{N_{P M T}} \frac{\left(O R_{i j}^{\mathrm{data}}-O R_{i j}^{\text {model }}\right)^{2}}{\left(\Delta O R_{i j}\right)^{2}+\sigma_{i j}^{2}}
$$

where $\Delta O R_{i j}$ is the statistical uncertainty in the occupancy ratio due to counting statistics and $\sigma_{i j}$ is an additional systematic uncertainty introduced to account for the PMT response variability as a function of the angle of incidence of the light.

\subsection{PMT Efficiencies Method}

The occupancy ratio method does not allow the PMT efficiencies $\epsilon_{i j}$ to vary in the fit, leaving the quantum efficiencies of the tubes unknown. According to [8], the efficiencies can be extracted from an optical scan, using the occupancy ratio fit results and the optical model intensity prediction, and used for another optical scan. This method provides a run-independent measurement of the QE of a PMT $j$ and does not depend on two run positions like the occupancy ratio method. The efficiency weighted occupancy $N_{i j}^{\text {model }}$ of a PMT $j$ in a run $i$ is then equivalent to the 
prompt intensity $N_{i j}^{\text {data }}$. The fit compares the data to the model in the same way:

$$
\chi^{2}=\sum_{i}^{N_{\text {run }}} \sum_{j}^{N_{P_{M T}}} \frac{\left(N_{i j}^{\text {data }}-N_{i j}^{\text {model }}\right)^{2}}{\left(\Delta N_{i j}\right)^{2}+\sigma_{i j}^{2}},
$$

which is more precise, assuming the error on the measurement of the efficiencies is small. The $\sigma_{i j}$ value is found via a polynomial function of the incident angle which takes into account the larger spread in PMT efficiencies at large incident angle [10]. Using this method, the efficiencies cannot be re-extracted. At the end, the PMT efficiencies method is less biased since the efficiencies are not dependent on the other optical parameters.

\subsection{Synopsis of this Thesis}

Optical calibration techniques and analyses are required to ensure that SNO can precisely measure the energies, positions, and directions of incoming solar neutrinos using optical and energy response functions. The SNO analyses select events according to a fiducial volume of radius $R_{\text {fid }}$ from the center; the volume is contained inside the AV volume to avoid the low energy radioactive background signal detected from the $\mathrm{H}_{2} \mathrm{O}$ volume. Similarly, the optically calibrated volume can be attributed a $R_{\text {fid }}^{\text {opt }}$ radius. To increase the precision of the optical response in the entire fiducial volume, data must be taken far away from the detector center and completely analyzed so that $R_{\text {fid }}^{\text {opt }} \gtrsim R_{\text {fid }}$.

In this thesis, a method to increase the optical volume radius $R_{\text {fid }}^{\text {opt }}$ is proposed, based on Monte Carlo data and on a simple detector geometry analytic model. The application of this method in the current or standard optical data processing 
enhances the way the data is selected before it is passed to the fit, for the data taken in the volume contained by the AV. The results presented in the following chapters have been processed and fitted using the occupancy ratio method of Section 1.4 only. The impact of the new technique is verified by looking at the most important optical parameters given by the occupancy ratio fit: the PMT angular response per incident angle and the media attenuation coefficients.

Chapter 2 describes the way the optical data is selected when processed. The data selection criteria explained are valid for the standard processing method of the pure $\mathrm{D}_{2} \mathrm{O}$ and salt phases of the experiment. Chapter 3 provides the details of the analytic model used in a larger $R_{\text {fid }}^{\text {opt }}$ analysis. Chapter 4 explains how the analytic model can be tested with a Monte Carlo simulation. Chapter 5 summarizes how the analytic model code is implemented and added to the actual optical data analysis software. Chapter 6 shows the fit results when the method is applied to the data of a laserball scan. There is also a comparison made with previous fits obtained with the current standard method. Chapter 7 is a discussion of the results and of the importance to further improve the optical calibration analyses and data processing methods for the future phases of SNO, in order to ensure the best precision when measuring the detector optical constants. 


\section{Chapter 2}

\section{Optical Data Selection}

This chapter describes the standard optical data selection process for the scans performed in the two first phases of SNO. It explains how the PMT occupancies are organized in a structure usable by the fitting program, which gives the optical model parameters. This selection is necessary to remove low or high occupancy PMTs that would affect the fit considerably and lead to unphysical optical constants.

Runs taken in the outer portion of the central volume, with source position radius $R_{S}$ of $450 \mathrm{~cm}$ and greater, are subject to more severe selection criteria than runs taken near the center. The reason is that the time spectrum of the photons detected resulting from those runs is not totally understood. A careful study of the PMT selection process combined to a time spectrum comparison on a run-by-run basis led to an improved understanding of the reasons why many PMTs are discarded for those runs. An enhanced data selection method is proposed to include more data in this region of the detector. 


\subsection{Optical Data Organization}

The statistics available in an optical data set depends on the total number of PMTs. Recall from Section 1.2 that there are $N_{P M T}$ entries representing each valid PMT for each run. Thus, for a typical scan containing $N_{\text {run }}$ runs, there are $N_{\text {data }}=$ $N_{\text {run }} \times N_{P M T}$ data points to be considered in the analysis. For a PMT $j$ associated with a run $i$, a series of flags, $f_{i j}$, exist to verify the availability of a specific PMT or its associated electronic channel before it is used. More than $80 \%$ of the PMTs are found to be valid for most runs.

\subsubsection{Analysis Tools}

The main software tools used for the optical data analysis are embedded in QOCA, the QSNO [11] optical data analysis module, and SNOMAN [12]. QOCA contains all the $\mathrm{C}++$ classes, written to be used with ROOT [13], necessary to manipulate and store the optical data. SNOMAN is the SNO Monte Carlo simulation and analysis program. It precisely reproduces the detector response under specific conditions.

QOCA contains many classes performing specific tasks. Briefly, QOptics calculates the light paths. Then, QPath uses QOptics to find the source positions, to calculate the occupancy ratios of Equation (1.6), and to store the data in a structure called a QOCATree. The QOCATree structure contains $N_{\text {run }}$ QOCARun's, each QOCARun contains $N_{P M T}$ QOCAPmt's.

QOptics is the principal class used for the optical calibration analyses. It loads the detector geometry and the PMT positions (as in SNOMAN), and determines the light paths from a given source position to all the PMTs of the detector. Given 


\begin{tabular}{|c|c|c|c|}
\hline \hline Flag $f_{i j}$ & Object & Reason & Verification \\
\hline \hline$f_{\text {belly }}$ & AV belly plate & Lensing & Light path \\
$f_{\text {neck }}$ & AV neck region & Absorption & Light path \\
$f_{\text {rope }}$ & AV support rope & Absorption & Light path \\
$f_{\text {pipe }}$ & D $_{2}$ O circulation pipe & Lensing & Light path \\
\hline$f_{\text {pavr }}$ & AV Reflectivity & Additional light & Relative position \\
\hline$f_{\text {basic }}$ & Electronic & PMT basic invalidity & Read banks \\
\hline$f_{\text {bad }}$ & None & Global PMT flag & Logical OR \\
$f_{\text {cbad }}$ & None & Global PMT flag (center) & Logical OR \\
\hline \hline
\end{tabular}

Table 2.1: PMT flags used in the optical data selection process. The objects may change the way the PMTs detect the incoming light. The reason why the flag is applied is given along with the verification made using the optical analysis software.

a specific PMT, the determination of the light paths in the different media of SNO leads QOptics to automatically find the distances, the times, the angles of the incident and transmitted light at the interfaces, the Fresnel transmission coefficients, etc.

\subsubsection{PMT Flags}

Table 2.1 contains the flags that can be applied to PMTs or to light paths between the source and the PMTs. The methods of QOptics check if the light paths traverse optically unclear objects. The objects (see Section 1.1) which can affect a regular light path are the equatorial belly plates, suspension ropes, water circulation pipes, and neck region. If the light path does cross these objects, within some tolerance, specific flags are raised. The flags are $f_{\text {belly }}, f_{\text {neck }}, f_{\text {rope }}$, and $f_{\text {pipe }}$, respectively. The light path then carries a bad flag, as interaction with these objects may result in lens- 
ing effects, abnormal scattering, or absorption of the incoming light. Consequently, the PMT might detect altered light and be flagged as a bad PMT. This selection is necessary because the optical model of Section 1.3 does not consider these unclear objects. Figure 2.1 shows an XSNOED [14] view of the PMTs flagged because of the first four reasons of Table 2.1 for a source at the center of the detector. The other flags of Table 2.1 are not applied based on light path calculations. They are explained below.

The $f_{\text {pavr }}$ flag is reserved for AV reflections. This flag is applied only if the relative position between the source and a PMT fulfills a certain condition: there is no path calculation. It discards PMTs that are subject to detect the reflected light, which adds an extra amount of light to the prompt light. This is discussed in more detail in Section 2.2 .

The $f_{\text {basic }}$ flag is an electronic channel flag. The electronic channels associated with the PMTs must be found in a good state; the channel calibration status (CHCS) bank contains information about the state of each channel. This information is based on the charge distribution for each PMT and the different discriminator walk for the different channel thresholds. Offline tubes are also identified in data acquisition (DQXX) banks. The flag is applied if the PMTs is already identified as bad in one of these banks: there is no path calculation necessary.

Finally, there are global flags that look at all the other flags. The $f_{\text {bad }}$ flag is applied to off-center runs and the $f_{\text {cbad }}$ flag is a similar flag for central runs. If only one flag is raised for any reason, the global flag is also raised. It provides a way to verify all the flags at once for a PMT. The global flags are not applied based on physical conditions: there is no path calculation. These flags perform a summary 


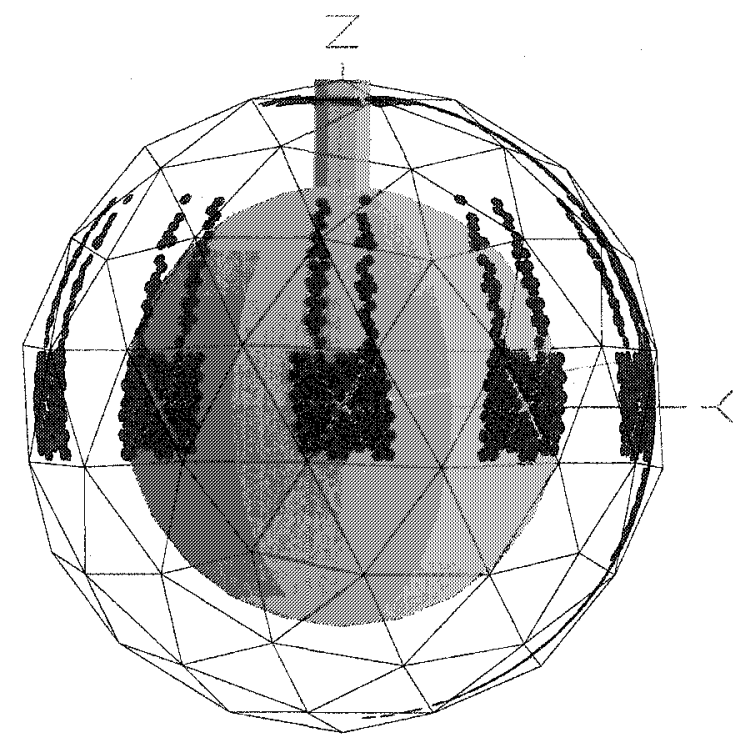

(a) Acrylic vessel inside the PSUP geodesic sphere.

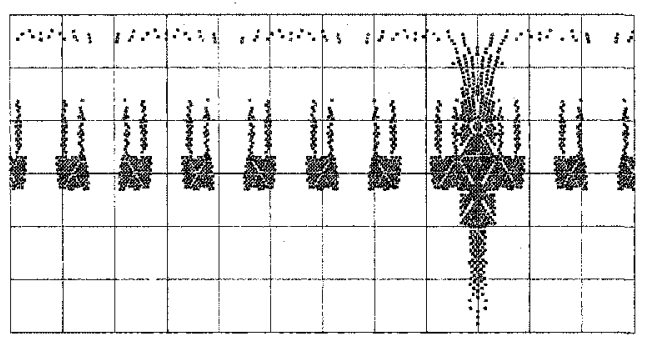

(b) Rectangular projection view.

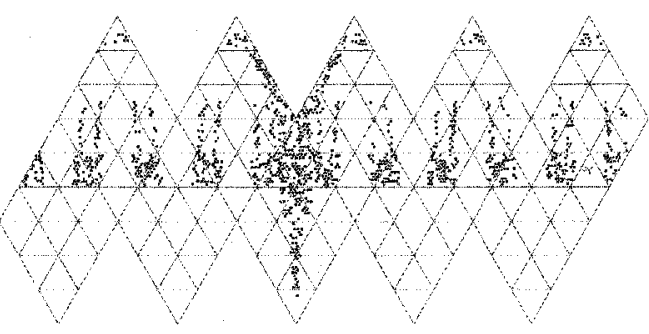

(c) Flat map view.

Figure 2.1: PMTs flagged by QOptics for a source at the center of the detector. Only the flags due to belly plates, ropes, circulation pipe, and AV neck are used. In this case, the belly plates and support ropes affect PMTs at the equator region of the PSUP sphere. The circulation pipe is going from the bottom to the top which explains the PMTs flagged along a vertical line. The PMTs flagged around the neck aperture are due to the non-transparent $A V$ neck region. For other source positions, the number of PMTs flagged is approximately the same but the PMTs are different. 
check, or a logical OR condition, of all the other flags.

\subsection{Prompt AV Reflections (PAVRs)}

Prompt AV reflections are reflections that can be treated as prompt light by mistake. The light path of a PAVR is very similar to the path of the prompt light. A PAVR and a prompt light photons are both detected by a PMT within the same time interval, which causes the prompt peak, used in the analysis of the SNO optical data, to be contaminated with an extra amount of light. This phenomenon only occurs when the source position is at large radius, close to the acrylic vessel. The runs with these source positions are called high radius runs $\left(R_{S} \geqslant 450 \mathrm{~cm}\right)$. Figure 2.2 shows the importance of having positions close to the AV. These runs allow a measurement of the high angle PMTR parameters. The incident angle of light on the PMT surface increases with the source radius $R_{S}$. As the source comes closer to the $\mathrm{AV}$, the light is detected at an incident angle of nearly $50^{\circ}$. The remainder of this chapter explains how the $f_{\text {pavr }}$ flag is applied and its influence on the selection process of the data of high radius runs.

\subsubsection{Default PAVR Selection Criterion}

The default QOptics code flag which takes into account possible contamination of the prompt peak by PAVRs $\left(f_{\text {pavr }}\right)$ is only verified for high radius runs:

$$
450 \mathrm{~cm} \leqslant R_{S} \leqslant 580 \mathrm{~cm} .
$$




\section{Maximum Incident Angle vs Source Radius}

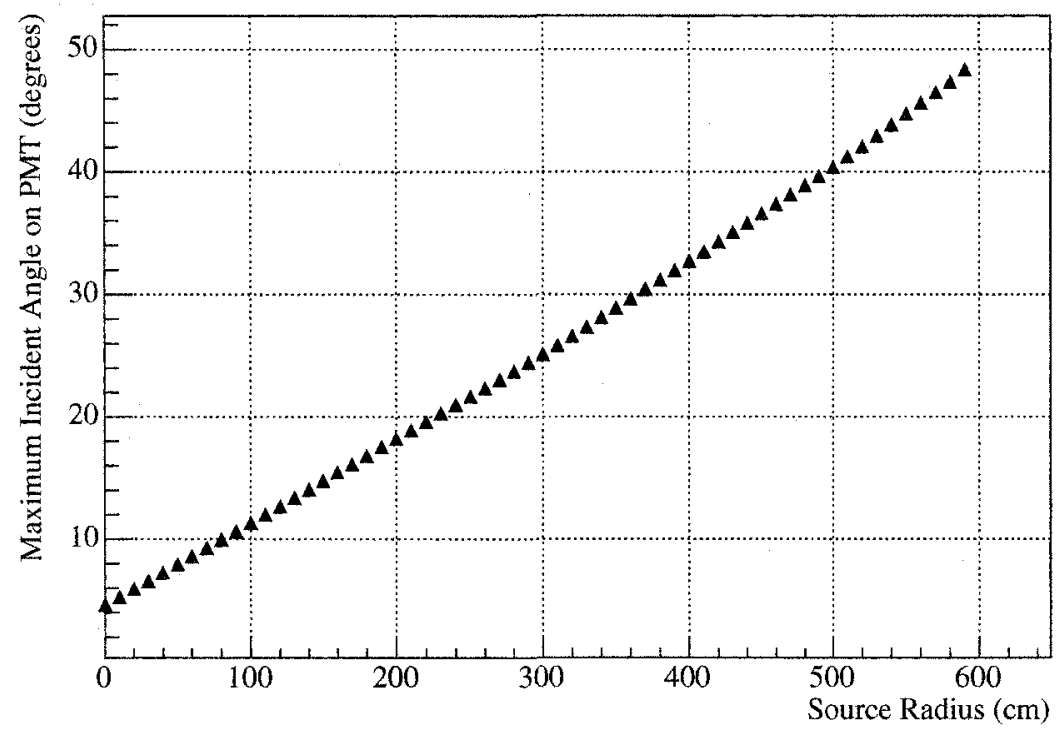

Figure 2.2: Maximum incident angle on PMT surfaces as a function of the source position radius. The $A V$ is at $600 \mathrm{~cm}$ from the center. The calculations were done with QOptics.

The QOptics function IsPromptAvRefI $(\mathrm{s}, \mathrm{p})$ looks at the source position vector $\vec{s}$ with magnitude $R_{S}$ from the center of the detector to the source and for the PMT vector $\vec{p}$ with magnitude $R_{P S U P}$ from the center to a given PMT. Figure 2.3 shows the two vectors. Let $\hat{s}$ and $\hat{p}$ be the unit vectors associated with $\vec{s}$ and $\vec{p}$, respectively, and $\theta_{p s}$ the angle between the two vectors. If the scalar product condition

$$
\hat{p} \cdot \hat{s} \equiv \cos \theta_{p s}<0.1
$$

is fulfilled, or,

$$
\theta_{p s}>1.47 \mathrm{rad} \simeq 85^{\circ}
$$




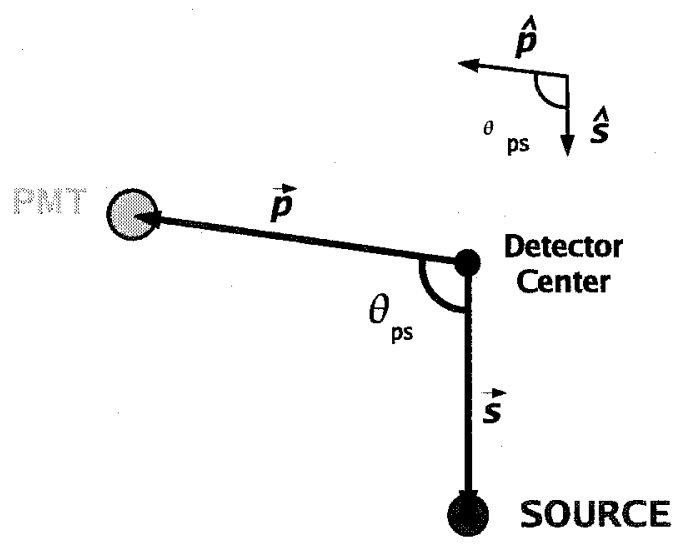

Figure 2.3: Vector representation of the source and the PMT positions within the detector.

the path is flagged and later the PMT is not analyzed or used in the fit. This default cut is also called the $85^{\circ}$ cut and is shown in Figure 2.4 for a source on the negative $z$-axis as displayed by XSNOED. The cut is insensitive to $R_{S}$ in the range defined by Equation (2.1).

Data from high radius runs with $R_{S}>500 \mathrm{~cm}$ are usually not included in the fit, since high occupancy tubes contained in these runs bias the fit for the PMTR, even with the $85^{\circ}$ cut described above. This is mainly caused by the PAVR contamination as the reflected light goes back to the $\mathrm{D}_{2} \mathrm{O}$ volume and is detected by other tubes with a short time delay. The time spectrum (PMT time distribution) of high radius runs must be understood before considering modifications which would improve the default selection criterion of Equation (2.2). 


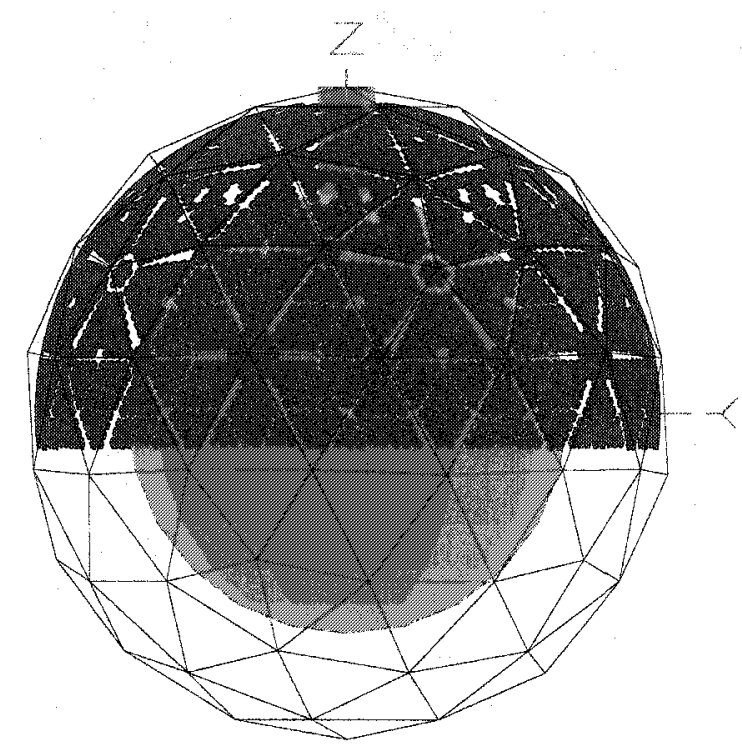

(a) Acrylic vessel inside the PSUP geodesic sphere.

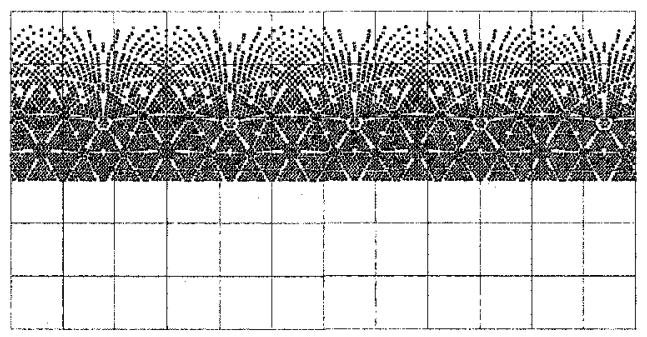

(b) Rectangular projection view.

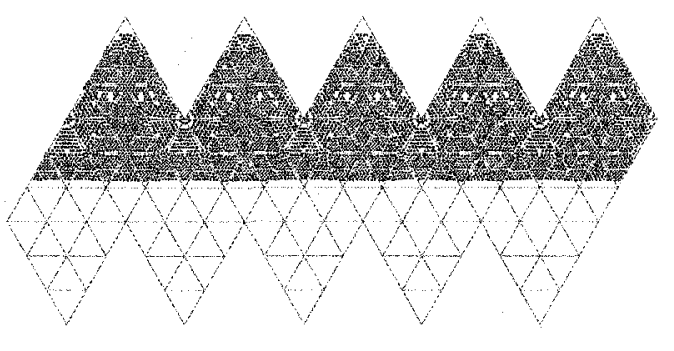

(c) Flat map view.

Figure 2.4: Example of PAVR cut for a source along the negative z-axis for any source position at high radius $\left(R_{S} \geqslant 450 \mathrm{~cm}\right)$. Only the PMTs flagged using the default $85^{\circ}$ cut are shown. More than $50 \%$ of the 9438 valid tubes are removed for a single high radius run. For this particular case, 5189 (55\%) PMTs are flagged. In principle, even more PMTs could be added with the action of other flags (see Figure 2.1). 


\subsubsection{Light Peaks}

Figure 2.5 shows a typical time distribution for a run taken at the center of the detector. It is also called a time residuals plot. The time values plotted have been corrected for the prompt peak central time of all the PMTs to be centered at $t=0$ ns. The time residual plots give global statistics of all the PMT hits added together, with no cut applied. The data selection is not done directly from these plots, but they do provide good diagnostics for a given run.

The peaks can be identified from Figure 2.5 since all the PMTs basically detect light at the same time (given the perfect spherical detector with the source at the center). The largest peak is the prompt light peak centered at $t=0$ ns. It is caused by the direct light. The smaller peaks on each sides are the PMT pre- and late-pulsing, respectively. The other peaks (for $t>40 \mathrm{~ns}$ ) are reflection peaks. The $\mathrm{AV}$ and PSUP reflections happen when the light reflects and travels back through the entire detector central volume to hit a PMT on the opposite side. The central run reflections are also called $180^{\circ}$ reflections. Since the PSUP is further from the source than the AV, the PSUP reflection peak comes after the AV reflection peak in time. The extra distance is $2 \times\left(R_{P S U P}-R_{A V}\right) \simeq 500 \mathrm{~cm}$ which corresponds approximately to 23 ns. For a central run, all the PMTs will detect these reflections. The importance of the reflection peaks compared to the prompt peak is non-negligible, but still small (about $1 \%$ ). The $35^{\circ}$ PMT reflector reflections are also important enough to be considered. The incident light comes at normal incidence on the PMT but hits the reflector and bounces back at $35^{\circ}$ with respect to the PMT normal towards another PMT.

Figure 2.6 shows similar time spectra for runs taken at a wavelength of $500 \mathrm{~nm}$ 


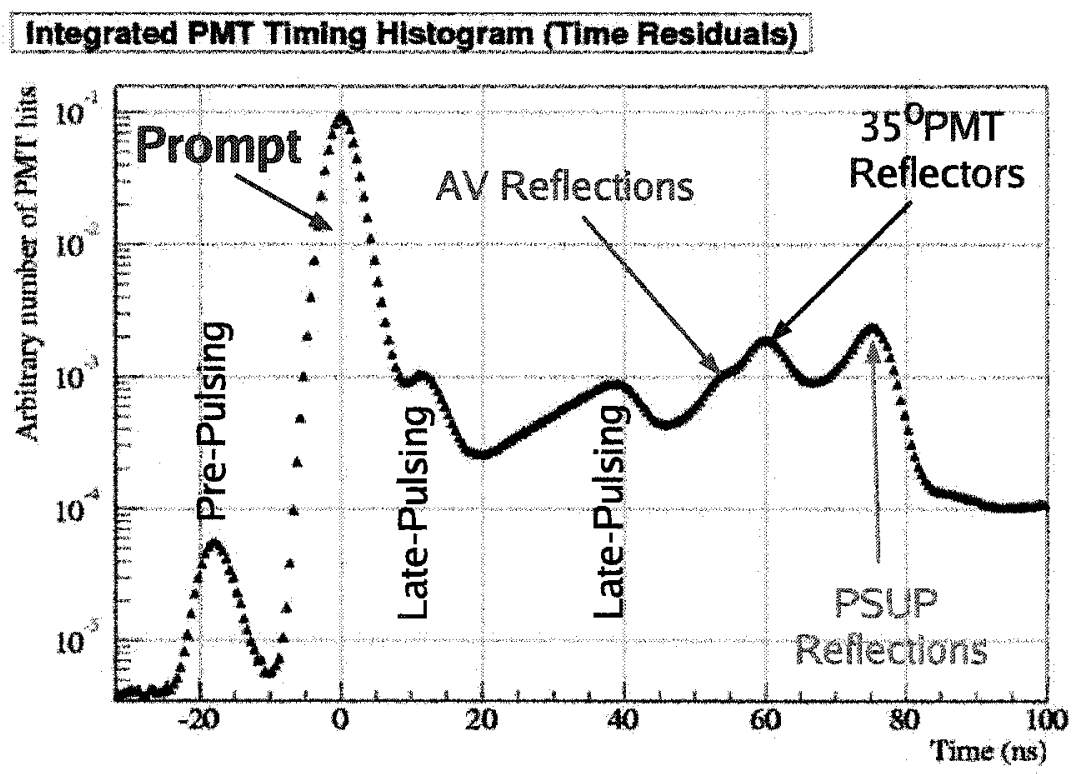

Figure 2.5: Example of a time distribution plot for a central run. The light peaks are identified.

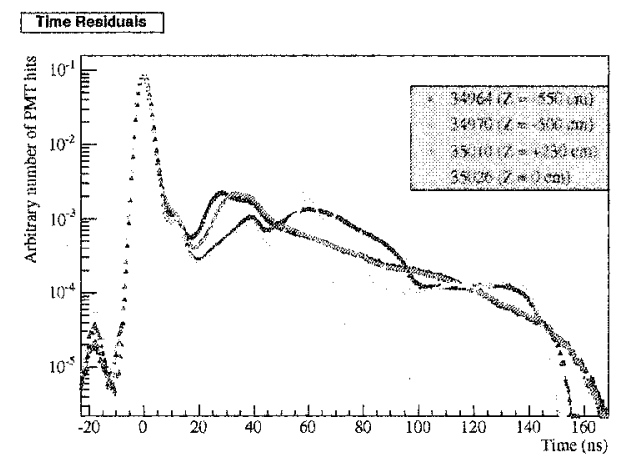

(a) All peaks.

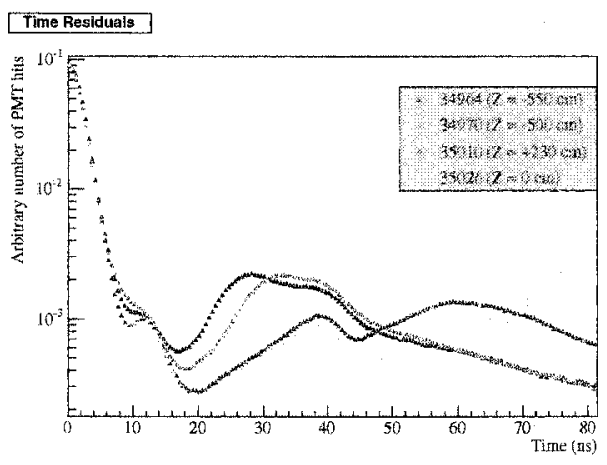

(b) Reflection peaks.

Figure 2.6: Time distribution plot for runs of the October 2003 scan. These runs were taken on the vertical central $z$-axis of the detector at $\lambda=500 \mathrm{~nm}$. View these plots in color. 
from the October 2003 scan. For each run, the source was positioned on the vertical $z$-axis of the detector, $(0,0, z)$. There is a central run $(35026)$ plotted as a reference. As the $z$ coordinate of the position increases, the radius $R_{S}=|z|$ increases and the reflection peaks decouple in time because the various reflected photons are detected at different times by different PMTs. The light peaks change in time and relative importance. This is a serious argument for the consideration of a PAVR selection criterion that depends on $R_{S}$.

The prompt peak used in the analysis is selected from the time residual plots with a time window, the QOCA prompt time window, that has a full width of $8 \mathrm{~ns}$. The contamination of the prompt peak happens when a reflection peak reaches the prompt peak. In Figure 2.6(b), the reflection tails for runs 34964 and 34970 overlap with the prompt peak. Between the prompt and the first late-pulsing peak, there is a shoulder indicating that the prompt peak tail and the reflection peak data add together in time. Knowing the AV is the closest object to the source at high radius, those reflections are caused by the AV.

\subsection{Motivation for a New PAVR Cut}

A new selection criterion combined with a better understanding of the AV reflections is necessary to include all the runs and fully analyze the optical data set of a scan. It is important to investigate how the AV reflections happen, determine if the probability of occurrence is significant, and verify if the effect is the same for all high radii values in the range determined by Equation (2.1). The time residual plots already showed that the reflection light peaks are changing in size and time 
as a function of $R_{S}$ compared to the prompt peak. Hence, the default PAVR cut should be replaced by an appropriate $R_{S}$-dependent selection criterion. The goal of that replacement is to improve the precision at which the QOCA analysis extracts the $\mathrm{D}_{2} \mathrm{O}$ and $\mathrm{H}_{2} \mathrm{O}$ attenuations, and the PMT angular response. The consequences of such a change are:

- the PMTR measurement in the range $\left[0^{\circ}, 40^{\circ}\right]$ of incident angle would most likely have more statistics and provide a more precise angular response parametrization of the PMTs,

- the runs with $R_{S}>500 \mathrm{~cm}$ would be included in the fit, such that new information in the range $\left[40^{\circ}, 50^{\circ}\right]$ could be measured with better precision,

- all the parameters from the optical model would be extracted for a larger fiducial volume, and

- possible bias in the calibration method could be identified from the previously inaccessible regions of the detector.

By keeping the actual format of the QOptics class, slight modifications of the criterion of Equation (2.2) would not change the way the optical data is processed. Equation (2.2) can be rewritten to show explicitly the limits of the scalar product:

$$
-1.0<\hat{p} \cdot \hat{s}<+0.1
$$


where the lower limit -1.0 means $\theta_{p s}=180^{\circ}$ (PMT on opposite side of the detector with respect to the source). These two cut limits are defined as follows:

$$
\begin{aligned}
& \cos \theta_{p s}^{\max }=-1.0, \\
& \cos \theta_{p s}^{\min }=+0.1 .
\end{aligned}
$$

This notation can be extended to a radius $\left(R_{S}\right)$ dependent criterion of the form:

$$
\cos \theta_{p s}^{\max }\left(R_{S}\right)<\hat{p} \cdot \hat{s}<\cos \theta_{p s}^{\min }\left(R_{S}\right) .
$$

This new condition is expected to give varying number of flagged PMTs for different runs with the same $R_{S}$ because of the different intrinsic PMT positions. It is caused by the fact that the PMTs lie on a geodesic structure, not a perfect sphere. This introduces an additional complication since one has to find the two limits for each high radius run. It ensures however that the flag $f_{\text {parr }}$ is applied in a general way.

In Chapter 3, an AV reflection analytic model is developed. The model is made such that the calculations of the radius dependent cut limits can be made within the QOptics class. In Chapter 4, the results of the analytic model are tested against Monte Carlo simulations. In Chapter 5, the AV reflection analytic model is inserted into the QOCA processing. In Chapter 6, measurements of $\cos \theta_{p s}^{\max }\left(R_{S}\right)$ and $\cos \theta_{p s}^{\min }\left(R_{S}\right)$ are made and replaced in the AV reflection QOptics flagging function, and the data of a selected scan is reprocessed with the new PAVR method. The impact of the changes on the fit for the optical constants is also summarized.

For post-optics data analysis, the optical response function is used for the determination of the detector energy response function. Since the optically cali- 
brated fiducial volume of the detector increases with the maximum source radius

$R_{\mathrm{fid}}^{\mathrm{opt}}=R_{S}^{\max }$, the entire fiducial volume will be optically calibrated ( $R_{\mathrm{fid}}^{\text {opt }}=R_{\mathrm{fid}}$ ) and the energy scale systematic uncertainties probably reduced. Finally, the impact on the PMTR and media attenuations measurements is discussed in Chapter 7. 


\section{Chapter 3}

\section{An Analytic Model for AV Reflections}

An analytic model was developed to verify if the AV reflections contaminate the prompt peak used for optical calibration analyses in the range $450 \leqslant R_{S} \leqslant 580 \mathrm{~cm}$. Understanding the PAVRs allows for changes in the data analysis of high radius runs, which in return allows a more accurate optical calibration of the detector. The analytic model presented in this chapter is an extension of the current path modelling contained in the QOptics code. An extensive description of light path modelling within SNO can be found in [15].

\subsection{Return to the Basics}

Consider light coming to an interface between two media. The light comes from an initial medium $m_{i}$, with an index of refraction $n_{i}$, at an angle of incidence $\theta_{i}$ with respect to the normal of the interface. Some transmitted light crosses the interface 
and enters medium $m_{t}$, with an index of refraction $n_{t}$, where the light leaves the interface at an angle of transmission $\theta_{t}$ with respect to the normal of the surface. The remaining amount of reflected light stays in medium $m_{i}$ and reflects with an angle of reflection $\theta_{r}$. Equations (3.1) and (3.2) are fundamental laws that govern the reflection, transmission, and refraction when light comes to an interface between two media:

$$
\begin{aligned}
\text { Law of reflection : } & \theta_{i} & =\theta_{r}, \\
\text { Law of refraction : } & n_{i} \sin \theta_{i} & =n_{t} \sin \theta_{t} .
\end{aligned}
$$

The amount of light reflected is found by multiplying the light intensity by the Fresnel reflection coefficients $R$ as described in [16]. The factor $R$ is found using the transmission coefficient, $T$, found from both parallel and perpendicular incident light polarizations:

$$
\begin{aligned}
t_{\|} & =\frac{2 n_{i} \cos \theta_{i}}{n_{t} \cos \theta_{i}+n_{i} \cos \theta_{t}} \\
t_{\perp} & =\frac{2 n_{i} \cos \theta_{i}}{n_{i} \cos \theta_{i}+n_{t} \cos \theta_{t}}
\end{aligned}
$$

such that

$$
R=1-T=1-\frac{1}{2}\left(\frac{n_{t} \cos \theta_{t}}{n_{i} \cos \theta_{i}}\right)\left(t_{\|}^{2}+t_{\perp}^{2}\right)
$$

where

$$
\cos \theta_{t}=\sqrt{1-\left(\frac{n_{i}}{n_{t}} \sin \theta_{i}\right)^{2}}
$$

is found using Equation (3.2).

The transmission coefficients are wavelength dependent since the refraction in- 


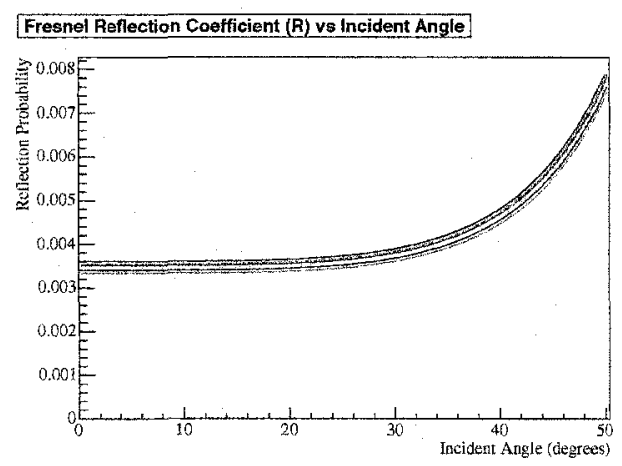

(a) Low incident angles.

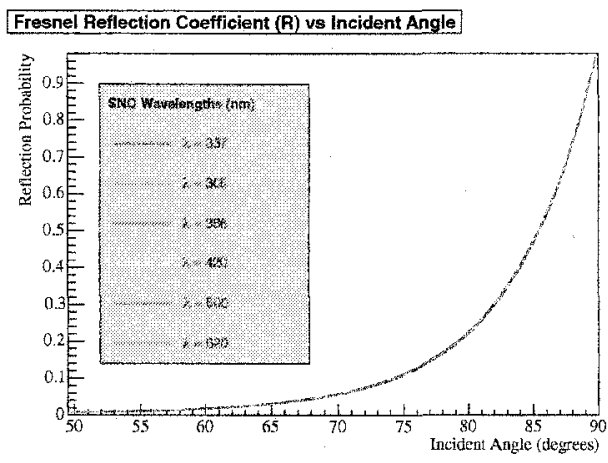

(b) High incident angles.

Figure 3.1: Fresnel reflection coefficient applied to the $D_{2} O-A V$ interface as a function of the incident angle on the $A V$. At low incident angles, the probability increases at around $35^{\circ}$ and a smaller difference exists between the various wavelengths. At high incident angles, the amount of reflected light becomes important.

dices for all SNO media are themselves functions of the wavelength. For the optical calibration of SNO, the source is inside the AV such that $m_{i}=\mathrm{D}_{2} \mathrm{O}, m_{t}=\mathrm{AV}$, $n_{i}=n_{\mathrm{D}_{2} \mathrm{O}}(\lambda)$, and $n_{t}=n_{A V}(\lambda)$. Figure 3.1 is a plot of $R$ versus the incident angle of light on the $\mathrm{AV}$ for $\lambda=337,365,386,420,500$, and $620 \mathrm{~nm}$. There is a small variation of $R$ with respect to the wavelength. For all wavelengths, the function increases rapidly at an incident angle of $35^{\circ}$.

Equations (3.1) to (3.5) can be used because the AV is approximated as a flat surface since its curvature is small. The second AV reflections that may occur on the outer AV surface (5.5 cm thick) are neglected. The multiple reflections happening at two different points on the AV (reflection of the reflected light) are also neglected based on the low value of the Fresnel reflection probability at small incident angles. 


\subsection{Analytic Model}

This section presents an analytic model which keeps track of the reflections on the $\mathrm{AV}$ for different source positions at low and high radii. The model consists of two concentric spheres: the AV and PSUP, which are both modelled, for simplicity, as perfect spheres with radii $R_{A V}=600 \mathrm{~cm}$ and $R_{P S U P}=845 \mathrm{~cm}$, respectively. To obtain reflections on the inner AV surface, source positions inside the $\mathrm{D}_{2} \mathrm{O}$ volume must be used: $0 \leqslant R_{S} \leqslant R_{A V}$. Three dimensional vector algebra and spherical coordinates define the positions of the source, the inner AV surface, and the PMTs. Straightforward calculations lead to radii, paths lengths, angles, etc. The symmetry of the detector about the $z$-axis is used to simplify the three dimensional model into a two dimensional model. This is done when verifying if the reflection condition defined by Equation (3.1) is fulfilled, assuming the incoming and reflected light are in the same plane.

\subsubsection{Model Geometry}

Figure 3.2 defines the important quantities used in the analytic model. The origin refers to the center of the detector. It corresponds to the common center of both AV and PSUP spheres. The following items are the main vectors, angles, and coordinate systems used:

$\vec{s} \quad$ The source vector from the origin to the source with magnitude $R_{S}$.

$\vec{p} \quad$ The PMT vector from the origin to the selected PMT with magnitude $R_{P S U P}$, which corresponds to the PSUP radius. 


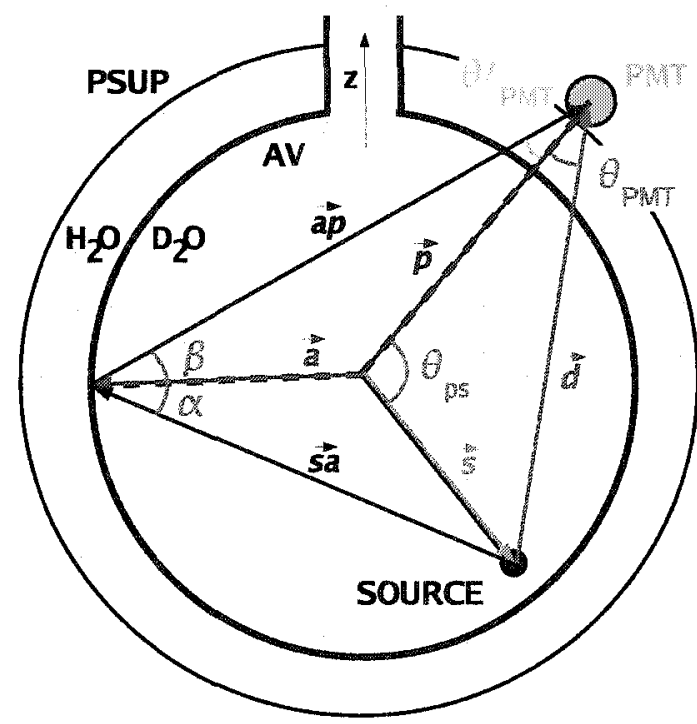

(a) Vector geometry.

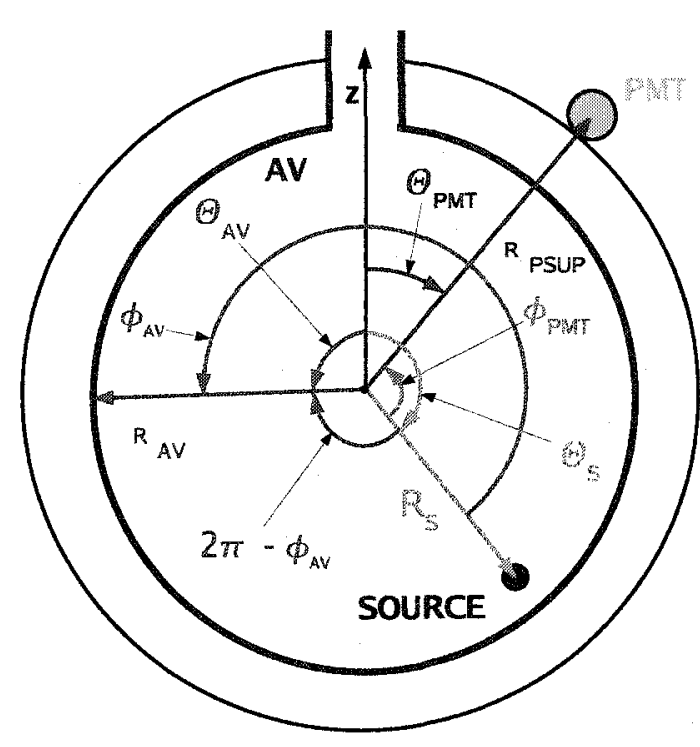

(b) Coordinate systems.

Figure 3.2: Vector geometry and coordinate systems used in the AV reflection analytic model. Dashed lines represent normals to the $A V$ reflection surface or to a PMT surface. Capital greek letters refer to spherical coordinates, small ones to the polar coordinates, except for $\theta$ reserved for angles between vectors. The source vector $\vec{s}$ defines $\phi=0$ in the polar plane. See texts for details. View this figure in color.

$\vec{a} \quad$ The AV vector from the origin to the inner AV surface with magnitude $R_{A V}$. It intercepts the AV reflection point.

$\vec{d} \quad$ The direct or prompt path vector from the source to the selected PMT with magnitude $r_{d}$, the distance traveled by the light directly from the source to the PMT. $\vec{d}=\vec{p}-\vec{s}$.

$\overrightarrow{s a} \quad$ The source-AV vector from the source to the AV reflection point with magnitude $r_{s a} . \overrightarrow{s a}=\vec{a}-\vec{s}$.

$\overrightarrow{a p} \quad$ The AV-PMT vector from the AV reflection point to the selected PMT with magnitude $r_{a p} \cdot \overrightarrow{a p}=\vec{p}-\vec{a}$. 
$\alpha \quad$ The incident angle of light from the source on the inner AV surface. Equivalent to $\theta_{i}$ of Equation (3.1). $\alpha=\arccos (\overrightarrow{s a} \cdot \vec{a})$.

$\beta \quad$ The reflection angle of reflected light from the AV reflection point to the selected PMT. Equivalent to $\theta_{r}$ of Equation (3.1). $\beta=$ $\pi-\arccos (\overrightarrow{a p} \cdot \vec{a})$.

$\theta_{p s} \quad$ The angle between the selected PMT vector $\vec{p}$ and the source vector $\vec{s}$, as defined in Chapter 2. $\theta_{p s}=\arccos (\vec{p} \cdot \vec{s})$.

$\theta_{P M T} \quad$ The incident angle of the prompt light on the selected PMT surface. $\theta_{P M T}=\arccos (\vec{p} \cdot \vec{d})$.

$\theta_{P M T}^{\prime} \quad$ The incident angle of the reflected light on the selected PMT surface. $\theta_{P M T}^{\prime}=\arccos (\vec{p} \cdot \overrightarrow{a p})$.

$r, \Theta, \Phi$ Spherical coordinate system. $\Theta$ is the polar angle used to define the angular position. The model is symmetric under $\Phi$.

$r, \phi \quad$ Polar coordinates system. $\phi$ is equivalent to the angular position $\Theta$, for $\Phi=$ constant in the spherical coordinate system. The source vector $\vec{s}$ defines $\phi=0$.

The model needs the source vector $\vec{s}$, the PMT vector $\vec{p}$, and the AV vector $\vec{a}$ to find all the relevant information.

\subsubsection{Derivations}

To apply the equations of Section 3.1 to the model, the reflections need to be ideal. The normal to the reflection surface, the incoming light, and the reflected light must be in the same plane. Using the source and the PMT vectors, and the detector center, a plane that contains these three objects can be constructed. This reduces 
the 3D spherical model, with coordinates $(r, \Theta, \Phi)$, to a 2D plane model, with polar coordinates $(r, \phi)$. Figure 3.2(b) explains the different coordinate systems used and how they are related. Knowing $\Theta_{S}$ and $\Theta_{P M T}$, the polar positions of the source and the PMT, other important parameters can be calculated using simple trigonometry. For instance, the various distances $\left(r_{d}, r_{s a}, r_{a p}\right)$, the incident and reflection angles $(\alpha, \beta)$, and the angular position of the AV reflection point $\Theta_{A V}$.

As an example, consider the case where the source is positioned along the negative $z$-axis $(x, y, z)=\left(0,0,-R_{S}\right)$, or in spherical coordinates $\left(R_{S}, \pi, 0\right)$. The source position fixes $\phi_{P M T}=\theta_{p s}$, which reduces the solution of an $\mathrm{AV}$ reflection to depend only on one variable, $\phi_{A V}$ :

$$
\begin{aligned}
\phi_{P M T} & =\Theta_{S}-\Theta_{P M T}=\pi-\Theta_{P M T}=\theta_{p s}, \\
\phi_{A V} & =\Theta_{S} \pm \Theta_{A V}=\pi \pm \Theta_{A V} .
\end{aligned}
$$

Due to the spherical symmetry of the model under $\Phi, \phi_{P M T}$ is restricted in the $\left[0^{\circ}\right.$, $\left.180^{\circ}\right]$ interval. However, $\phi_{A V}$ is a standard polar angle allowed in the interval $\left[0^{\circ}\right.$, $360^{\circ}$. The plus or minus sign is used to differentiate the $\mathrm{AV}$ positions that have the same $\Theta_{A V}$ but that are not similar from the PMT position point of view. Found from the law of cosines, Equations (3.9) to (3.13) define all the needed quantities in terms of the known parameters $R_{S}, R_{A V}, R_{P S U P}$, and $\phi_{P M T}$, and the only unknown parameter $\phi_{A V}$.

$$
\begin{aligned}
r_{d} & =\sqrt{R_{S}^{2}+R_{P S U P}^{2}-2 R_{S} R_{P S U P} \cos \phi_{P M T}} \\
r_{s a} & =\sqrt{R_{A V}^{2}+R_{S}^{2}-2 R_{A V} R_{S} \cos \phi_{A V}}
\end{aligned}
$$




$$
\begin{aligned}
r_{a p} & =\sqrt{R_{A V}^{2}+R_{P S U P}^{2}-2 R_{A V} R_{P S U P} \cos \left(\phi_{P M T}-\phi_{A V}\right)} \\
\alpha & =\arccos \left\{\frac{R_{A V}-R_{S} \cos \phi_{A V}}{r_{s a}}\right\} \\
\beta & =\arccos \left\{\frac{R_{A V}-R_{P S U P} \cos \left(\phi_{P M T}-\phi_{A V}\right)}{r_{a p}}\right\} .
\end{aligned}
$$

It is important to note the following dependencies. In Equation (3.12), $\alpha$ depends on the radius of the source, $R_{S}$, and on the angular position of the reflection point, $\phi_{A V}$ : $\alpha \equiv \alpha\left(R_{S}, \phi_{A V}\right)$. Similarly, in Equation (3.13), $\beta$ depends on the angular position of the selected PMT, $\phi_{P M T}$, and also on the angular position of the reflection point, $\phi_{A V}: \beta \equiv \beta\left(\phi_{P M T}, \phi_{A V}\right)=\beta\left(\theta_{p s}, \phi_{A V}\right)$.

\subsubsection{Solutions}

The values assigned to $R_{S}$ and $\phi_{P M T}$ are the input parameters in the algorithm which finds numerically the $k$ solution(s) $\phi_{A V}^{k}$ to Equation (3.14) or (3.1):

$$
\alpha^{k}\left(R_{S}, \phi_{A V}^{k}\right)=\beta^{k}\left(\phi_{P M T}, \phi_{A V}^{k}\right)
$$

These analytic functions are plotted as a function of the AV angular positions in Figure 3.3. The ordinate gives $\alpha$ and $\beta$ in degrees, and the abscissa, the position of the reflection points on the $\mathrm{AV}$ in degrees, $0^{\circ}$ being the negative $z$-axis, increasing counterclockwise on the polar plane as explained in Figure 3.2(b). The $k$ solution(s) for each set of parameters $\left(R_{S}, \phi_{P M T}\right)$ are the values $\phi_{A V}^{k}$ where both curves cross each other $(\alpha=\beta)$. The time differences $\Delta t^{k}$ between a reflection light path $k$ and the prompt light path can then be calculated using the distances in the different media and the group velocities. Referring to Figure 3.3, Table 3.1 gives an example 


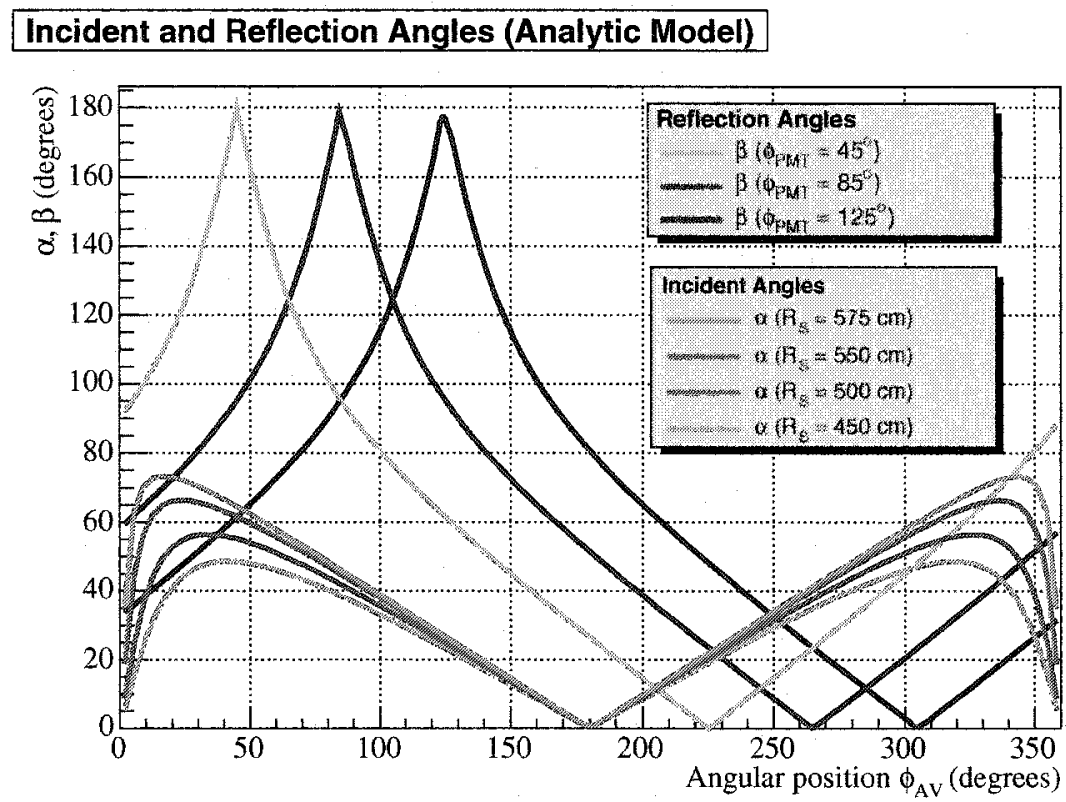

Figure 3.3: Analytic functions of the incident and reflection angles on the AV surface. Each curve corresponds to a specific configuration as shown in the legend. The reflection solutions allowed by the model are those where both curves cross each other $(\alpha=\beta)$. View this plot in color.

of parameters that leads to four $\mathrm{AV}$ reflection solutions $(k=4)$. The number of solutions is not always four. It varies as the input parameters $R_{S}$ and $\phi_{P M T}$ change. These specific case solutions have different incident angle on the AV surface $(\alpha)$. The solutions are distinguished using the $\alpha$ value and the position of the AV reflection point $\phi_{A V}$.

For $k=1$, the light has a relatively high incident angle on the $\mathrm{AV}, \alpha^{(1)}=39^{\circ}$. The reflection happens at the bottom of the $\mathrm{AV}, \phi_{A V}^{(1)}=8^{\circ}$, such that the reflected light follows the prompt light by a small amount of time. The reflected light in this case strikes the PMT with nearly the same incident angle than the prompt light. The reflection is characterized by $\overrightarrow{s a} \cdot \vec{d}<0$; a solution that fulfills that condition and $\alpha \leqslant 40^{\circ}$ is called a low incidence (LI) reflection. 


\begin{tabular}{|c||c|c|c|c|}
\hline \hline \multicolumn{5}{|c|}{$R_{S}=500 \mathrm{~cm}, \phi_{P M T}=125^{\circ}$} \\
\hline \hline$k$ & 1 & 2 & 3 & 4 \\
\hline$\alpha^{k}$ & $39^{\circ}$ & $56^{\circ}$ & $32^{\circ}$ & $30^{\circ}$ \\
$\phi_{A V}^{k}$ & $8^{\circ}$ & $37^{\circ}$ & $252^{\circ}$ & $352^{\circ}$ \\
\hline \hline
\end{tabular}

Table 3.1: Example of analytic AV reflection solutions for a specific set of input parameters. The solution can also be found graphically on Figure 3.3.

For $k=2$, the light has an high incident angle on the $\mathrm{AV}, \alpha^{(2)}=56^{\circ}$. The reflection happens between the bottom and the equator of the $\mathrm{AV}, \phi_{A V}^{(2)}=37^{\circ}$, such that the reflected light follows the prompt light by a small amount of time. The reflected light in this case strikes the PMT with a greater incident angle than the prompt light. The reflection is characterized by $\overrightarrow{s a} \cdot \vec{d}>0$; a solution that fulfills that condition and $\alpha>40^{\circ}$ is called a high incidence (HI) reflection.

For $k=3$, the light has a small incident angle on the AV, $\alpha^{(3)}=32^{\circ}$. The reflection happens on the opposite side of the $\mathrm{AV}$ with respect to the PMT, $\phi_{A V}^{(3)}=$ $252^{\circ}$, such that the reflected light follows the prompt light by a large amount of time. The reflected light in this case strikes the PMT with nearly the same incident angle than the prompt light, but on the other side of the PMT normal. The reflection is characterized by $\overrightarrow{s a} \cdot \vec{d} \geqslant 0$; a solution that fulfills that condition and $\alpha \leqslant 45^{\circ}$ is called a far $(\mathrm{F})$ reflection. The limit of $45^{\circ}$ is determined by the geometry of the model.

Finally, for $k=4$, the light has a small incident angle on the $\mathrm{AV}, \alpha^{(4)}=30^{\circ}$. This reflection is an unphysical solution given by the model. It is a consequence of the spherical symmetry of the model in which the functional forms of Equations (3.12) and (3.13) allow complete rotation $\left(360^{\circ}\right)$ of both $\phi_{P M T}$ and $\phi_{A V}$. The unphysical 


\begin{tabular}{|ccc|}
\hline \hline Reflection Name & Meaning & Limits \\
\hline \hline Low Incidence & Low incident angle & $\alpha \leqslant 40^{\circ}$ \\
(LI) & on the AV surface & $\hat{s a} \cdot \hat{d}<0$ \\
\hline High Incidence & High incident angle & $\alpha>40^{\circ}$ \\
(HI) & on the AV surface & $\hat{s} a \cdot \hat{d}>0$ \\
\hline Far & Opposite side & $\alpha \leqslant 45^{\circ}$ \\
$(\mathrm{F})$ & of the detector & $\hat{s a} \cdot \hat{d} \geqslant 0$ \\
\hline Unphysical & Not real & None \\
$(\mathrm{U})$ & & $\hat{s a} \cdot \hat{a p}=-1$ \\
\hline \hline
\end{tabular}

Table 3.2: Analytic model $A V$ reflection solutions and names. All the valid PMTs of the SNO geometry will at least experience the far $A V$ reflection for any value of $R_{S}$ within the $\mathrm{D}_{2} \mathrm{O}$ volume. The scalar products give an additional way of recognizing the reflections given the geometry of the model.

solution corresponds to $\alpha=\beta$ found on the same side of the normal to the plane (light reflected in exact opposite direction). It is characterized by $\hat{s a} \cdot \hat{a p}=-1$; a solution that fulfills that condition is called an unphysical (U) reflection.

Table 3.2 summarizes the four possible reflection solution types and the quantities used to identify them. All the configurations allow the F solution, which would be found at any value of $R_{S}$. Example situations containing all the reflections are shown in Figure 3.4.

\subsubsection{Probabilities}

For HI reflections, the reflected light continues in the same direction. These reflections are just deflections and they have large incident angles on the AV, causing them to be non-negligible according to the reflection probability function of Figure 3.1. For LI and F reflections, the reflected light bounces back and the light goes in 


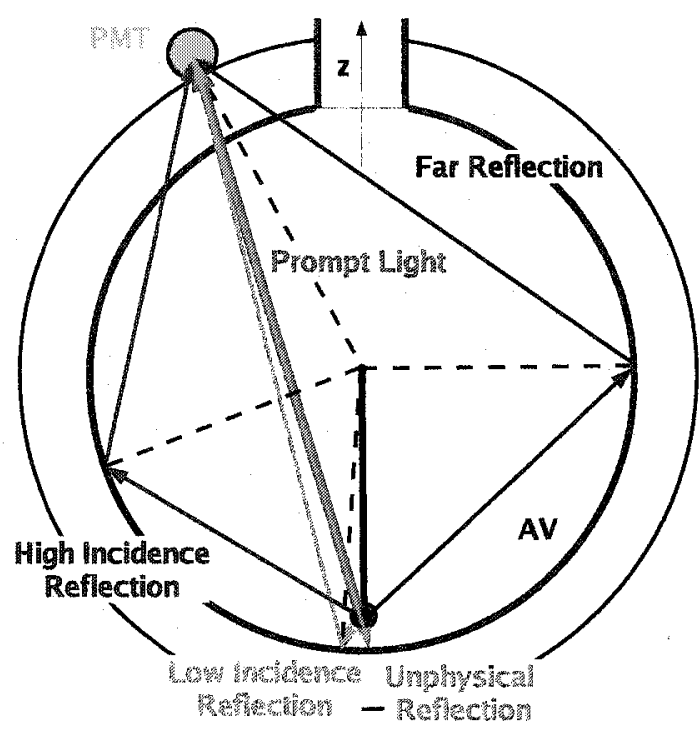

(a) Top PMT.

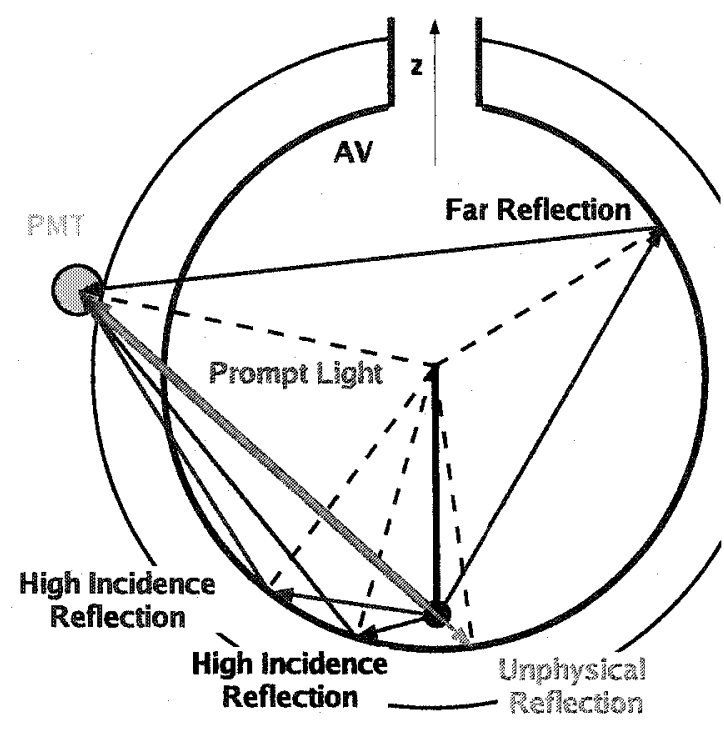

(b) Equatorial PMT.

Figure 3.4: Reflections found with the analytic model for PMTs located at the top and near the equator of the detector. The time difference between the reflected and the prompt light can be visually evaluated by the area between the prompt light vector and the reflection vector in those specific cases. The larger the area, the longer the delay. Note that this rule is not general. Note that for PMTs near the equator, the $L I$ reflection becomes a second $H I$ reflection. View this figure in color.

the opposite direction. The LI reflections are prompt light followers. Even if their probability is lower than HI reflections, their path length difference with the prompt light is usually very small making them good candidates for contamination. The $\mathrm{F}$ reflections can have an incident angle on the AV up to $45^{\circ}$ but because of the large distance that the reflected light has to go through to reach a PMT on the other side of the detector, they are not likely to be detected. 


\subsection{Merging the Analytic Model with the Exist- ing Optics}

A way to employ this analytic model with QOptics is to write a separate class. This was done at Carleton University in a private version of the QOCA code compiled against the default official QSNO version [11]. The new class inherits all the methods in QOptics to calculate distances, angles, paths, etc., but contains additional methods to numerically solve for a physical AV reflection given the source and a PMT positions. The new class's name is QOCAReflect. The QOCAReflect code is a natural extension of QOptics: it not only follows the prompt photons, but also the photons that reflect on the inner AV surface that may contaminate the prompt light peak. The class requires two parameters:

1. A three dimensional source vector, $\vec{s}$, from which the source position radius, $R_{S}$, in cm, is automatically calculated and passed as the $R_{S}$ parameter.

2. A time window, $t_{w}$, in ns. It is an acceptance time difference parameter, used to activate the flag $f_{\text {pavr }}$ for PMTs that fulfill the condition $\Delta t^{k}<t_{w}$, where $\Delta t^{k}$ is the time difference calculated by QOptics between the reflected light path and the prompt light path.

The code uses the analytic model functions of $\alpha$ and $\beta$ to find where the reflections happen on the AV surface. It then uses QOptics to set a fake source at that AV reflection point to calculate the extra path lengths. Since the merged code works on a PMT-by-PMT basis, a loop over all the PMT for a given source position returns all the information about relative time of flights and transmission coefficients of such 


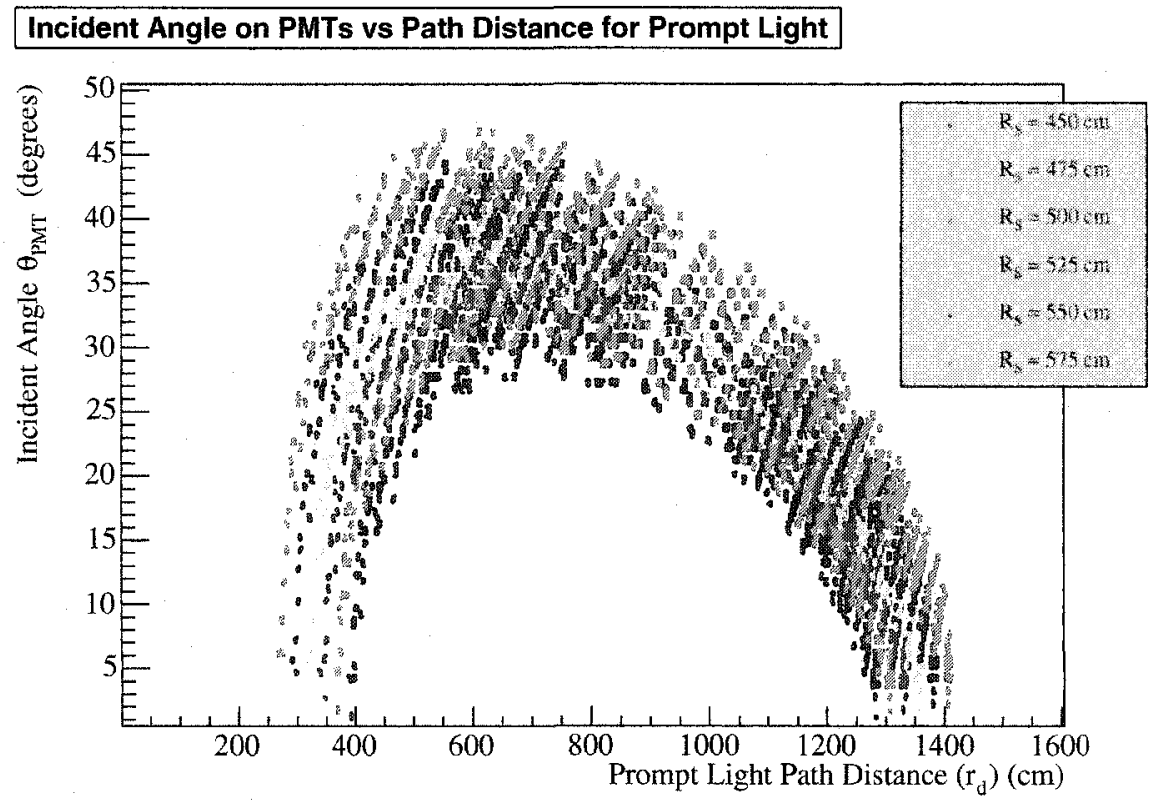

Figure 3.5: Scatter plot of the incident angle on the PMT surface versus the prompt light path for six source positions for the 9438 valid PMTs. High incident angle PMTs are neither close nor far but at average distance from the source. View this plot in color.

reflections. This code was tagged and released in the QSNO version 20.0 .

\subsubsection{Transmitted Light Information}

The QOCAReflect class allows data storage such that a complete diagnostic of a high radius position can be done before proceeding to the optical data processing. The transmitted light information is given by QOptics and can be stored as well. Figure 3.5 shows the prompt light incident angle on the PMT, $\theta_{P M T}$, as a function of the total prompt light path length, $r_{d}$, for the $R_{S}=450,475,500,525,550$, and $575 \mathrm{~cm}$. It shows that the PMT incident angle reaches a maximum for prompt path lengths between 450 and $600 \mathrm{~cm}$. Figure 3.6 shows, with XSNOED maps, that these PMTs are located between the equator and the bottom of the detector for a 
source on the negative $z$-axis. The positions shown are $(x, y, z)=(0,0,-500)$ and $(0,0,-550)$. The PMTs closer and further to the source see the prompt light at almost $0^{\circ}$ of incidence.

\subsubsection{Reflected Light Information}

QOCAReflect enables the user to fill specific diagnostic histograms every time a specific quantity is calculated for a PMT. Table 3.3 shows six source position radii chosen to look at the newly available reflected light path information in the $R_{S}$ range of interest. The information of Table 3.3 is a summary obtained after a complete loop over the valid PMTs. Two time windows have been used for comparison: $t_{w}=8$ $\mathrm{ns}$ and $t_{w}=10 \mathrm{~ns}$. The determination of the time window value used in the analysis is discussed in Chapter 5. The number of reflections, $N_{r}$, refers to the total number of reflection solutions found. This number is independent of the time window. The maximum number of physical reflection solutions is $k=3$. This leads to a maximum number of calls $k \times N_{P M T}=28,314$ in QOCAReflect. For each position of Table 3.3 , all the PMTs are checked to find the $\cos \theta_{p s}$ limits, $\cos \theta_{p s}^{\max }$ and $\cos \theta_{p s}^{\min }$, based on $\Delta t^{k}<t_{w}$, as these limits are time window dependent. The number of bad PMTs affected by PAVRs, $N_{\text {bad }}$, depends on the $\cos \theta_{p s}$ limits and is also time window dependent. The larger the time window, the lower the acceptance, the larger the number $N_{\text {bad }}$. The default selection criterion of Figure 2.4 p.26 discards PMTs between $\cos \theta_{p s}^{\max }=-1.0$ and $\cos \theta_{p s}^{\min }=+0.1$ resulting in $N_{\mathrm{bad}}=5189(54.9 \%)$ in the range $450 \leqslant R_{S} \leqslant 580 \mathrm{~cm}$. Most positions in Table 3.3 give fewer $N_{\text {bad }}$ PMTs. There is an increase of the number of good PMTs with the new PAVR selection criterion. Above $R_{S}=550 \mathrm{~cm}$, the new PAVR cut agrees with the default cut such 


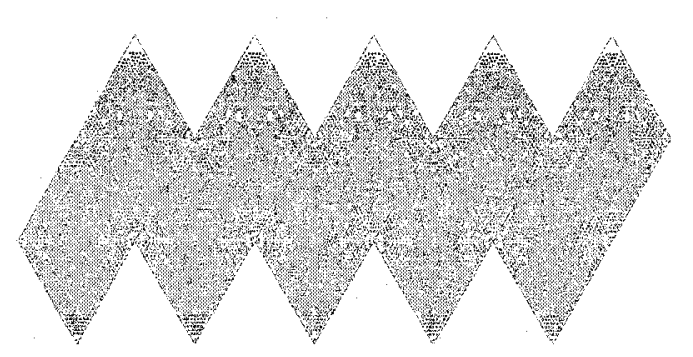

(a) Flat map view for $R_{S}=500 \mathrm{~cm}$.

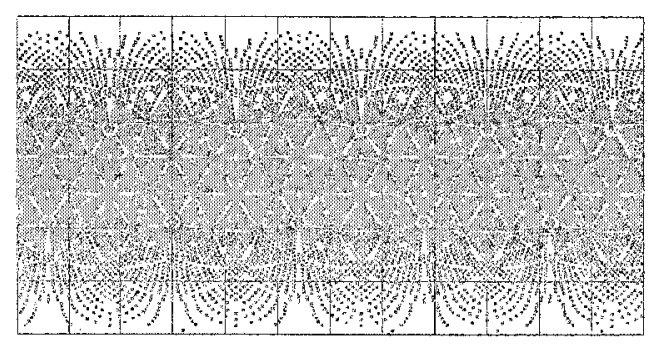

(c) Rectangular projection view for $R_{S}=500 \mathrm{~cm}$.

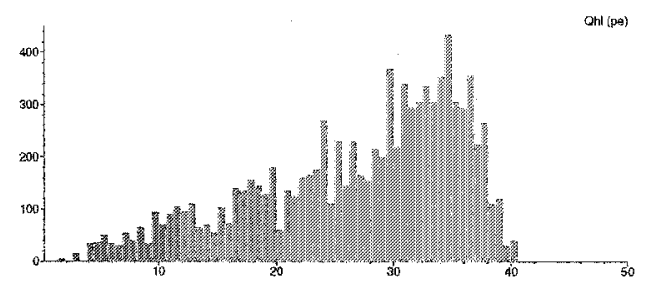

(e) $\theta_{P M T}$ distribution (in degrees) for $R_{S}=500 \mathrm{~cm}$.

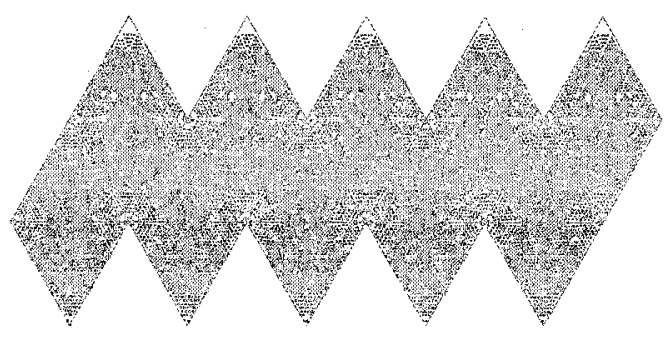

(b) Flat map view for $R_{S}=550 \mathrm{~cm}$.

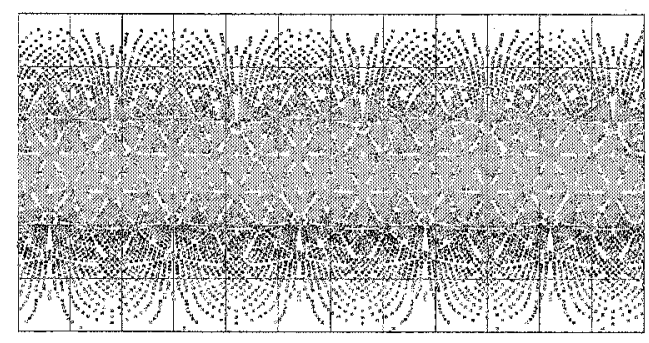

(d) Rectangular projection view for $R_{S}=550 \mathrm{~cm}$.

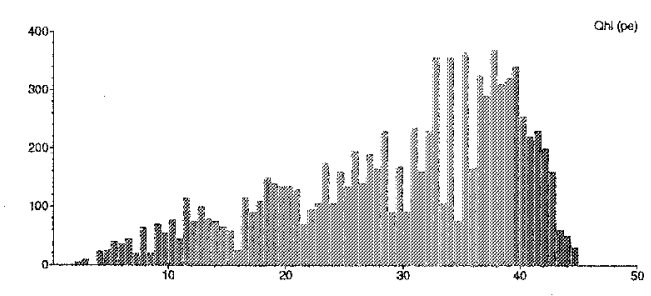

(f) $\theta_{P M T}$ distribution (in degrees) for $R_{S}=550 \mathrm{~cm}$.

Figure 3.6: Incident angle on the PMT surface distributions for sources at $(x, y, z)$ $=(0,0,-500)$ (left) and $(0,0,-550)$ (right) $\mathrm{cm}$. The detector equator lies on the central horizontal line for the flat map and the rectangular projection views. View these plots in color. 


\begin{tabular}{|cccccc|}
\hline$R_{S}(\mathrm{~cm})$ & $N_{r}$ & $t_{w}(\mathrm{~ns})$ & $\cos \theta_{p s}^{\max }$ & $\cos \theta_{p s}^{\min }$ & $N_{\text {bad }}(\%)$ \\
\hline \hline 450 & 12570 & 8 & - & - & $0(0)$ \\
& & 10 & - & - & $0(0)$ \\
\hline 475 & 13760 & 8 & - & - & $0(0)$ \\
& & 10 & -0.735 & -0.528 & $968(10.2)$ \\
\hline 500 & 15100 & 8 & -0.714 & -0.389 & $1548(16.3)$ \\
& & 10 & -0.988 & -0.389 & $2824(29.9)$ \\
\hline 525 & 16720 & 8 & -0.988 & -0.217 & $3634(38.4)$ \\
& & 10 & -0.988 & -0.217 & $3634(38.4)$ \\
\hline 550 & 18630 & 8 & -0.988 & -0.029 & $4589(48.5)$ \\
& & 10 & -0.988 & -0.029 & $4589(48.5)$ \\
\hline 575 & 21060 & 8 & -0.988 & +0.235 & $5804(61.4)$ \\
& & 10 & -0.988 & +0.235 & $5804(61.4)$ \\
\hline \hline
\end{tabular}

Table 3.3: QOCAReflect information for six source positions on the negative z-axis. The source position is $(x, y, z)=\left(0,0,-R_{S}\right)$ for the $R_{S}$ value given. $N_{\text {bad }}$ indicates the number of PMTs that fulfill $\Delta t^{k}<t_{w}$. The percentage value is the comparison to the number of transmissions (valid PMTs). When no $\cos \theta_{p s}$ values are found, it means that the data can be used without any cut for PAVR. Note that the $\cos \theta_{p s}^{\max }$ only gets to -0.988 since in this particular configuration the exact value -1.0 is in the neck aperture, where no PMTs exist.

that the number of flagged PMTs found at $R_{S}=575 \mathrm{~cm}$ is about $55 \%$.

Figure 3.7 shows the incident angle of the light that reflects on the AV. The integration of the $\alpha$ distribution for each $R_{S}$ gives the value $N_{r}$. HI reflections dominate the statistics of the $\alpha$ distributions as $R_{S}$ increases.

Figure 3.8 shows the incident angle of the reflected light on the PMTs, $\theta_{P M T}^{\prime}$, as a function of the reflected light path length, $r_{s a}+r_{a p}$, for $R_{S}=450,475,500,525$, 550 , and $575 \mathrm{~cm}$. This figure demonstrates that the reflected light has also access to PMTs with high incident angles in a reasonably short distance. The gap at 1800 


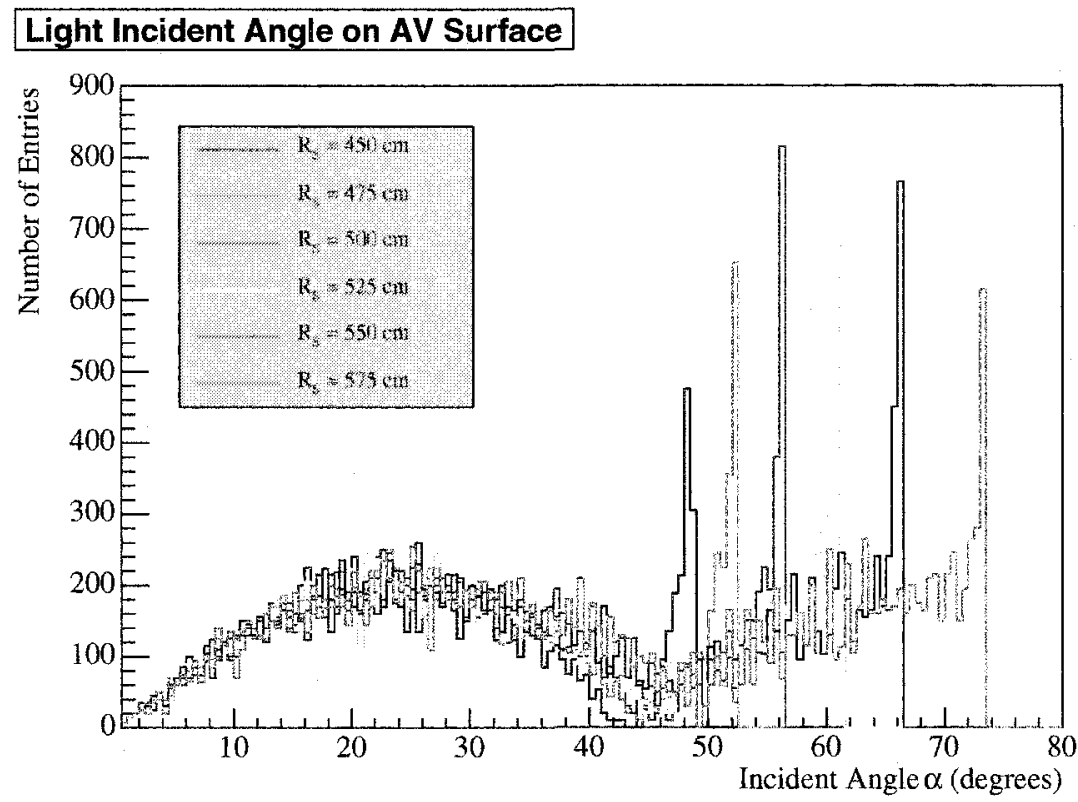

Figure 3.7: Angle of incidence on the inner AV surface as a function of the source position radius. Far reflections dominate the region below $45^{\circ}$. High incidence reflections are responsible for the peaks at high incident angles. There is no $\cos \theta_{\text {ps }}$ cut applied. View this plot in color.

$\mathrm{cm}$ separates the $\mathrm{F}$ reflections (larger distance) from the LI and HI reflections.

Figures 3.9 and 3.10 show the time differences $\Delta t^{k}$ versus the PMT position $\cos \theta_{p s}$ for the same source radius values for time windows of 8 and $10 \mathrm{~ns}$. In each plot of Figure 3.9, there are three curves representing each of the three reflections for each position. The top curve represents $F$ reflections, the middle curve the HI reflections, and the bottom curve the LI reflections. The F reflections exist in the time difference range $[25,120] \mathrm{ns}$, and the $\mathrm{HI}$ and $\mathrm{LI}$ reflections in the range $[0,25]$ ns. For $\cos \theta_{p s} \lesssim 1, F$ reflections from the opposite side of the detector strike PMTs close to the source. The reflections are the same as the $180^{\circ} \mathrm{AV}$ reflections described in Section 2.2, Figure 2.5. The $\mathrm{F}$ reflections cover all the range in $\cos \theta_{p s}$ since all the PMTs are subject to detect those reflections at any position. In Figure 3.9, HI 


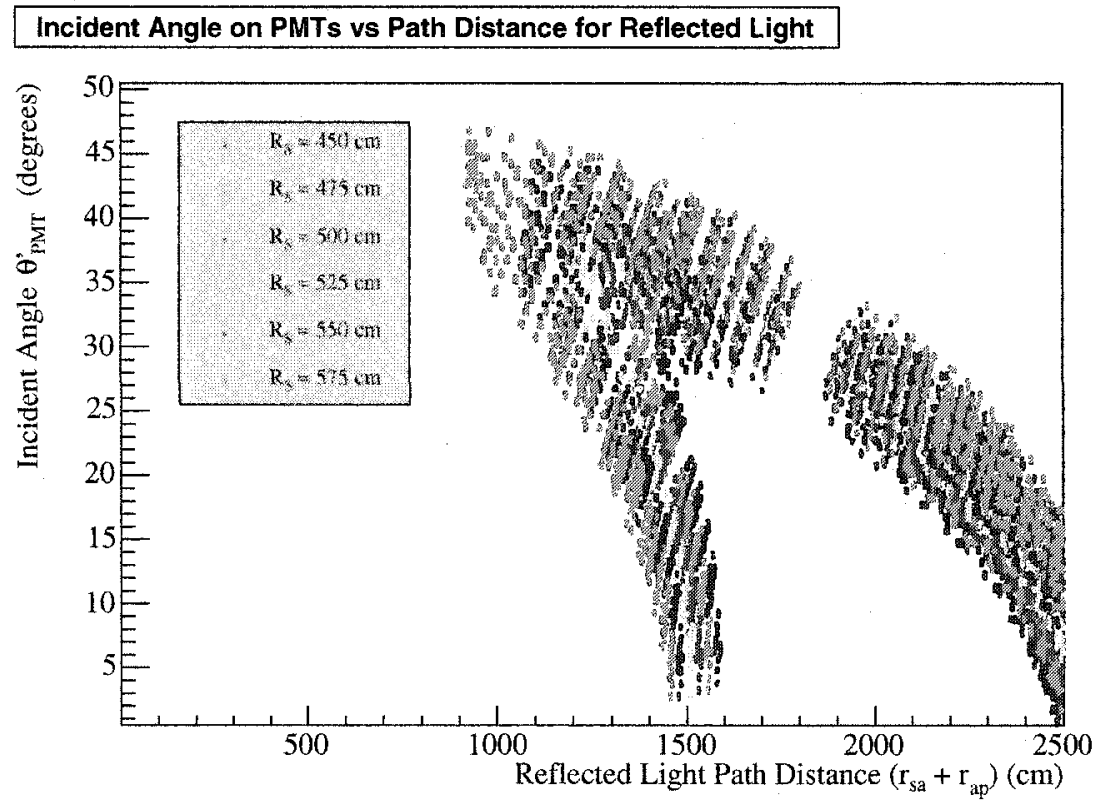

Figure 3.8: Scatter plot of the incident angle on the PMT surface versus the reflection light path for six source positions for a maximum of three reflections for each of the 9438 valid PMTs. High incident angle PMTs are close to the source viewed by the reflections such that they may interfere with the PMTR measurements if there is contamination of the prompt peak. There is no $\cos \theta_{p s}$ cut applied. View this plot in color.

and $\mathrm{F}$ reflections almost meet at $\cos \theta_{p s} \simeq-1$. For negative $z$-axis sources, the gap between HI and F reflections represent the neck region where there is no PMT. For a perfect $\mathrm{AV}$ sphere, there should be no gap between the curves at $\cos \theta_{p s}=-1$. HI and LI reflections also meet at some value of $\cos \theta_{p s}$; this value changes with the source radius $R_{S}$. At this $\cos \theta_{p s}$, the LI are considered as HI, based on the definitions of Table 3.2 .

In Figures 3.9 and 3.10 , the red color indicates that the time difference $\Delta t$ was smaller than the time window parameter $t_{w}$, the cases where the $f_{\text {pavr }}$ flag is set. The $\cos \theta_{p s}$ limits found in Table 3.3 correspond to the minimum and maximum values of $\cos \theta_{p s}$ shown in red in the figures. When no red points are found, as in Figure 
3.9 (a), it means no cut has to be made for PAVRs. Then no $\cos \theta_{p s}$ limits appear in Table 3.3. It can be seen by comparing the same source positions in Figures 3.9 and 3.10 that the time window parameter affects the number of flagged PMTs. The increase of the $t_{w}$ parameter increases the number of potential reflections that can reach the PMTs within the time window. Hence, there are less PMTs flagged for $t_{w}=8 \mathrm{~ns}$.

\subsection{Summary}

A simple geometrical analysis finds the physically allowed AV reflections for given source and PMT positions. The analytic model was inserted in the optics program to accurately calculate the extra light paths of the reflected light. The distances, angles of incidence, and time delays were extracted to understand the different solutions given by the analytic model.

Chapter 4 is a verification of the model predictions using Monte Carlo simulations. In Chapter 5 , it is explained how the time window acceptance $t_{w}$ is set before QOCAReflect is inserted in the optical data processing code. These steps lead to the comparison of the fit of the reprocessed data to the standard fit results of the October 2003 scan in Chapter 6. 


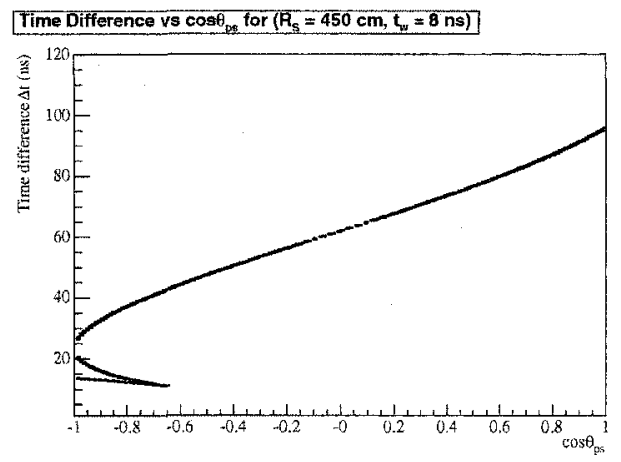

(a) $R_{S}=450 \mathrm{~cm}, t_{w}=8 \mathrm{~ns}$.

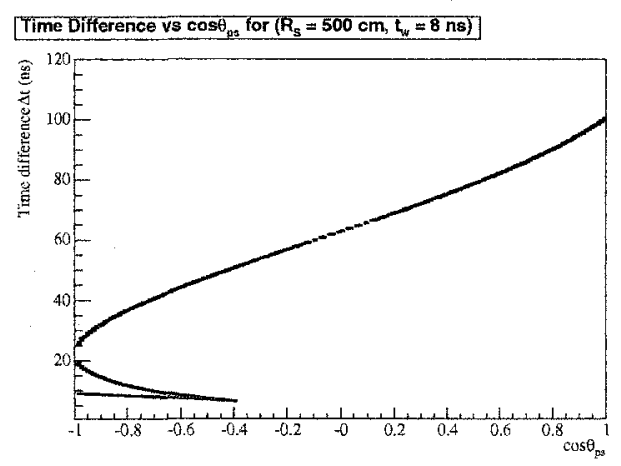

(c) $R_{S}=500 \mathrm{~cm}, t_{w}=8 \mathrm{~ns}$.

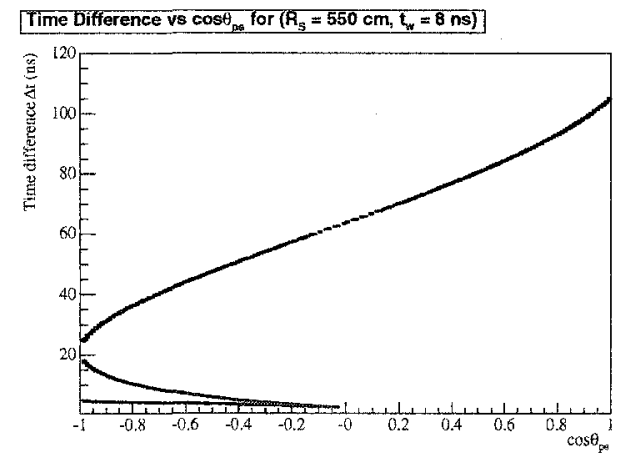

(e) $R_{S}=550 \mathrm{~cm}, t_{w}=8 \mathrm{~ns}$.

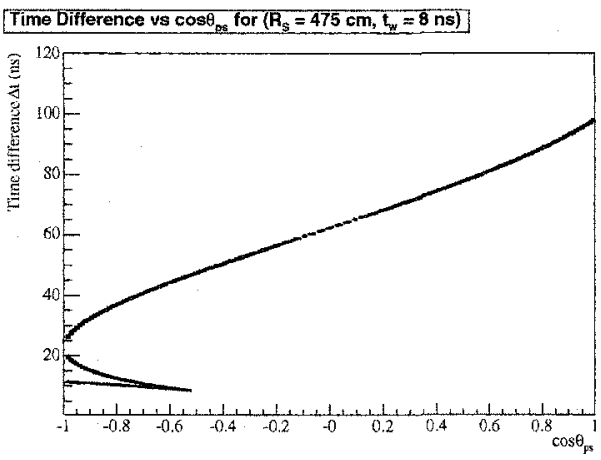

(b) $R_{S}=475 \mathrm{~cm}, t_{w}=8 \mathrm{~ns}$.

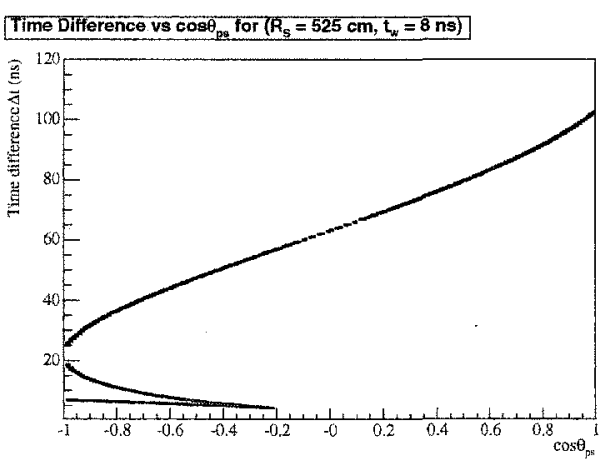

(d) $R_{S}=525 \mathrm{~cm}, t_{w}=8 \mathrm{~ns}$.

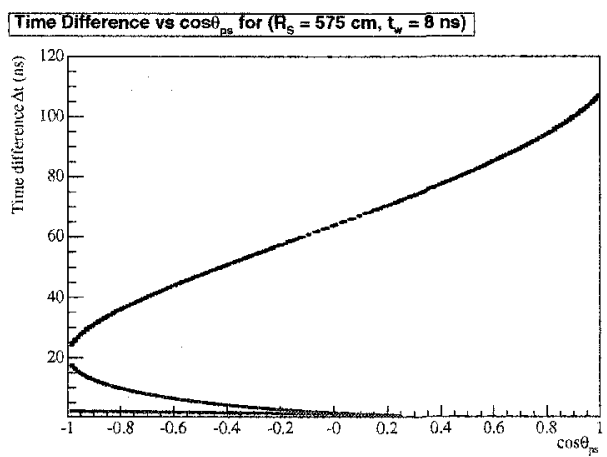

(f) $R_{S}=575 \mathrm{~cm}, t_{w}=8 \mathrm{~ns}$.

Figure 3.9: Scatter plot of the time differences $\Delta t$ between the reflection and prompt light paths versus $\cos \theta_{p s}$ for six source positions for a maximum of three reflections for each of the 9438 valid PMTs. Red data points indicate the positions of the contaminated PMTs that detect reflected light in a shorter time delay than the time window $t_{w}=8$ ns imposed. View these plots in color. 


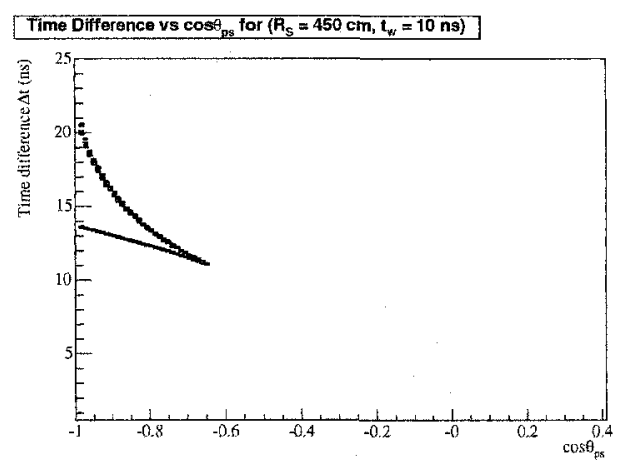

(a) $R_{S}=450 \mathrm{~cm}, t_{w}=10 \mathrm{~ns}$.

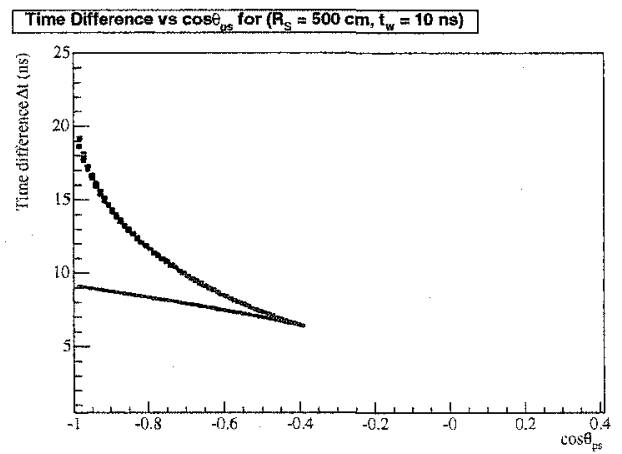

(c) $R_{S}=500 \mathrm{~cm}, t_{w}=10 \mathrm{~ns}$

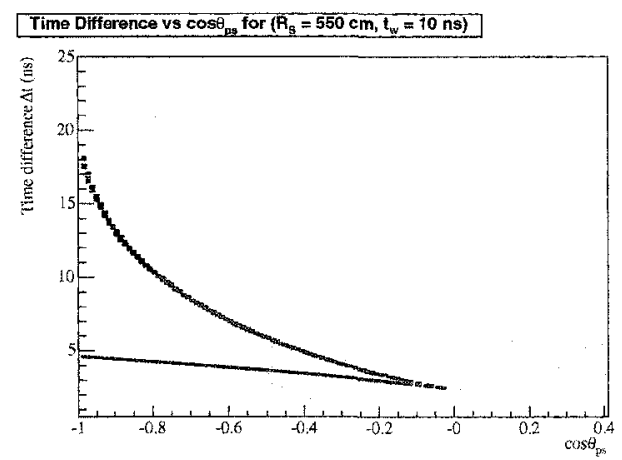

(e) $R_{S}=550 \mathrm{~cm}, t_{w}=10 \mathrm{~ns}$.

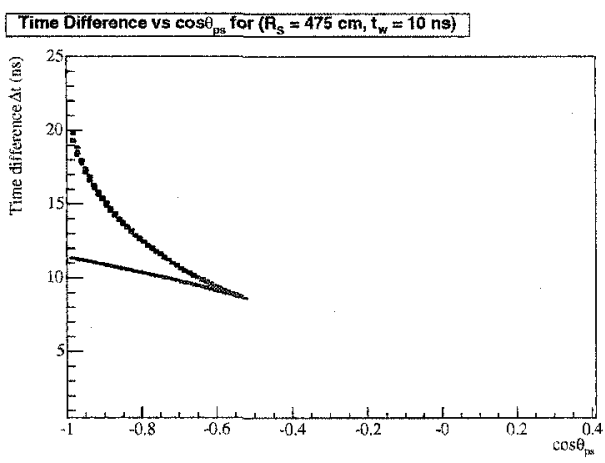

(b) $R_{S}=475 \mathrm{~cm}, t_{w}=10 \mathrm{~ns}$.

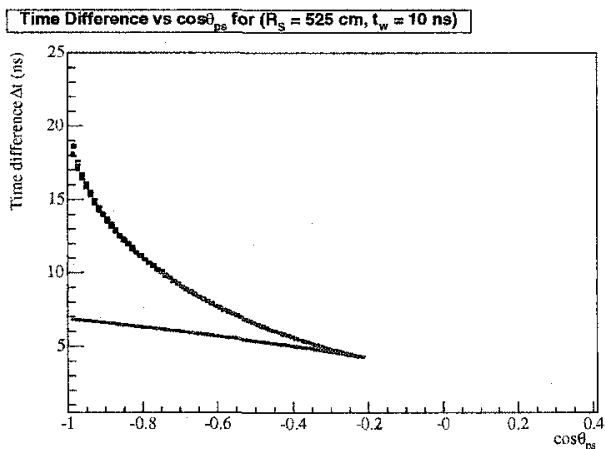

(d) $R_{S}=525 \mathrm{~cm}, t_{w}=10 \mathrm{~ns}$.

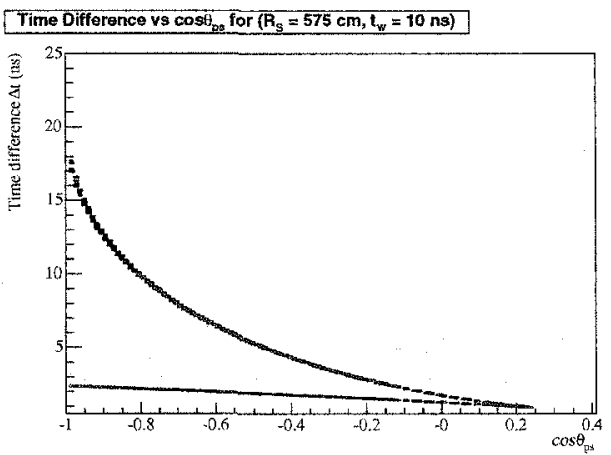

(f) $R_{S}=575 \mathrm{~cm}, t_{w}=10 \mathrm{~ns}$.

Figure 3.10: Scatter plot of the time differences between the reflection and prompt light paths versus $\cos \theta_{\text {ps }}$ for six source positions for a maximum of two reflections for each of the 9438 valid PMTs. Only low and high incidence reflection regions are shown. Same as Figure 3.9 but with a time window of $t_{w}=10 \mathrm{~ns}$ imposed. Since the time window is larger than in Figure 3.9, it gives more time for the reflected light to reach the position of a PMT, causing the cut limits on $\cos \theta_{p s}$ to eliminate more tubes. View these plots in color. 


\section{Chapter 4}

\section{Monte Carlo Checks}

It is important to check the validity of a simple analytic model with a detailed simulation of the detector. The reflected light information given by the analytic model contained in QOCAReflect must be compared to similar calculations performed by SNOMAN, the SNO Monte Carlo simulation program. The analytic part of the model resides in the way the reflection on the AV sphere is found and how the time difference between the reflected and the prompt light reaching a given PMT is calculated. Comparison with Monte Carlo (MC) PMT time distributions using different simulated monoenergetic sources is enough to confirm the model accuracy and precision.

\subsection{Introduction}

In order to verify if the reflected light in SNOMAN behaves as predicted by the analytic model, only the time information has to be extracted from the SNOMAN output or the ROOT calibration histograms (RCH). The RCH timing information is 
contained in a two dimensional histogram; on one axis the logical channel numbers $n$ associated to PMTs, on the other axis, the triggered time $t$ of all the photons detected (referred as hits) in a simulation run. In these histograms, the time $t$ is not corrected as it was in the time residual plots of Chapter 2: it is the raw time. The selection of a particular $n$ gives access to a single PMT channel time information. When there are more than one events, the PMT time information is given as a PMT time distribution. The projection of the two dimensional histogram on the logical number $n$ axis gives the number of photons detected by a particular PMT. Similarly, the projection on the time $t$ axis gives the time distribution of the entire PMT array, the sum of all the individual PMT time distributions.

\subsubsection{Time Information}

A $\mathrm{MC}$ event is the generation of a certain number of photons by the simulated calibration source. For each event, there is a time trigger synchronized with each photon leaving the source. The PMT hits appearing in the RCH histograms are caused by the source photons or by their reflections. The trigger time of about $100 \mathrm{~ns}$ is large enough to include the detection of the reflected light. A simulation or a MC run contains a certain number of events. In general, the number of hits registered by the PMTs does not correspond to the total number of photons since the generated photons can end up anywhere in the detector and be lost.

In a MC run, there must be enough photons detected by the PMTs such that the prompt and the reflected light hits form peaks as a function of time allowing the mean and width of the raw time distribution to be computed. Assuming the peaks are Gaussian, each peak $i$ has an amplitude $\mathrm{N}_{i}$, a mean time $\mu_{i}$, and a width $\sigma_{i}$ that 
characterize the raw time distribution:

$$
g_{i}(t ; n)=\mathrm{N}_{i}^{n} \exp \left\{-\left(\frac{t-\mu_{i}^{n}}{\sqrt{2} \sigma_{i}^{n}}\right)^{2}\right\}
$$

where the index $i$ serves to identify the peaks ( $i=$ prompt or ref) and the index $n$ identifies the PMT channel. The MC run equivalent of Equation (4.1) includes the contribution of all the PMTs:

$$
g_{i}(t)=\mathrm{N}_{i} \exp \left\{-\left(\frac{t-\mu_{i}}{\sqrt{2} \sigma_{i}}\right)^{2}\right\}=\sum_{n=1}^{N_{P M T}} g_{i}(t ; n)
$$

The central value $\mu$ and the width $\sigma$ of the peaks are found by fitting the Gaussian function of Equations (4.1) or (4.2) to the time distribution.

The primary information of interest is the time difference that separates the reflected light from the prompt light detected by a single PMT $n$ extracted from a simulated experiment. It is not straightforward to compare the time values obtained from simulations to the analytic model prediction because their absolute time reference, $t_{0}$, might be different. Usually, the time reference $t_{0}$ is defined as the source emission time. The prompt light is detected at $t_{\text {prompt }}=t_{0}+\mu_{\text {prompt }}^{n}$, and the reflected light at $t_{\mathrm{ref}}=t_{0}+\mu_{\mathrm{ref}}^{n}$. For the analytic model, the time reference $t_{0}$ is unknown. The absolute time difference $\Delta t_{\text {model }}$ is calculated by subtracting the prompt time $t_{\text {prompt }}$ to the reflected time $t_{\text {ref }}$, such that the unknown $t_{0}$ cancels. In the simulated data, unlike the time residual plots of Chapter 2, the prompt light peak does not always set $t_{0}=0$ ns. The MC time data must be manipulated to get an equivalent time difference with no unknown time reference. To obtain such a time difference, the PMTs detecting both prompt and reflected light must be identified, 
and their prompt and reflection peaks characterized with Equation (4.1). Then the mean time and the width of the peaks are used to calculate the time difference and its uncertainty:

$$
\begin{aligned}
\Delta t & =\mu_{\mathrm{ref}}^{n}-\mu_{\mathrm{prompt}}^{n} \\
\sigma_{\Delta t} & =\sqrt{\left(\sigma_{\mathrm{ref}}^{n}\right)^{2}+\left(\sigma_{\mathrm{prompt}}^{n}\right)^{2}} .
\end{aligned}
$$

$\Delta t$ is the time difference value obtained with $\mathrm{MC}$ data comparable to the analytic model time difference $\Delta t_{\text {model }} \equiv t_{\text {ref }}-t_{\text {prompt }}$.

\subsubsection{Light Production}

SNOMAN enables the user to choose a source by specifying a source type in a command file [12]. The source type is specified by a position type and direction type. The position type is related to the source size and shape: sphere, disk, or point. The direction type tells the light how to escape from the source: it can be a focused beam, isotropic light, or Čerenkov light.

For the purposes of this study, only monochromatic light sources are used. These monochromatic sources are called laser sources. A Gaussian shaped function is used to generate the time dependence of the laser pulses. Photon beams and bombs are both used to simulate events. Laser beams need to be assigned a beam direction, with spherical coordinates $(\theta, \phi)$, in addition to their source positions. Laser bombs only need to be assigned a position as the light is emitted isotropically from that position. 


\subsection{Laserdisk Source}

The first tests performed with a simple laser beam were not instructive. The beam source (direction type) was selected as a point source (position type) emitting light along one direction. Since the distance between neighboring PMTs on the PSUP is relatively large (PMT coverage of 59\%), most of the directions chosen did not give many PMT hits. The difficulty was to get PMT hits from both prompt and reflected light associated with a narrow beam.

To correct for this, a new non-point source was created to mimic the laser beam; this new position type is a disk-shaped source. The so-called laserdisk source has been implemented by Ranpal Dosanjh at Carleton [17]. An example of command file used to run the laserdisk source is given in Appendix A. The disk radius has to be set, defining the disk surface size. The photons are generated uniformly on the disk surface, hence creating a directed cylindrical beam. The direction of the beam can be independently selected from the disk orientation: the light can come out of the disk surface with an angle. The laserdisk source parameters are:

- the disk center position $(x, y, z)$, which sets the position radius $R_{S}$,

- the disk radius $R_{D}$,

- the disk orientation $\left(\theta_{\text {disk }}, \phi_{\text {disk }}\right)$, and

- the beam orientation $\left(\theta_{\text {beam }}, \phi_{\text {beam }}\right)$.

Figure 4.1 shows an example of a laserdisk source, its position, orientation, and photons generated from the surface. Light paths and expected reflection detection regions are also shown. Figure 4.2 shows an example of the PMT hits obtained 


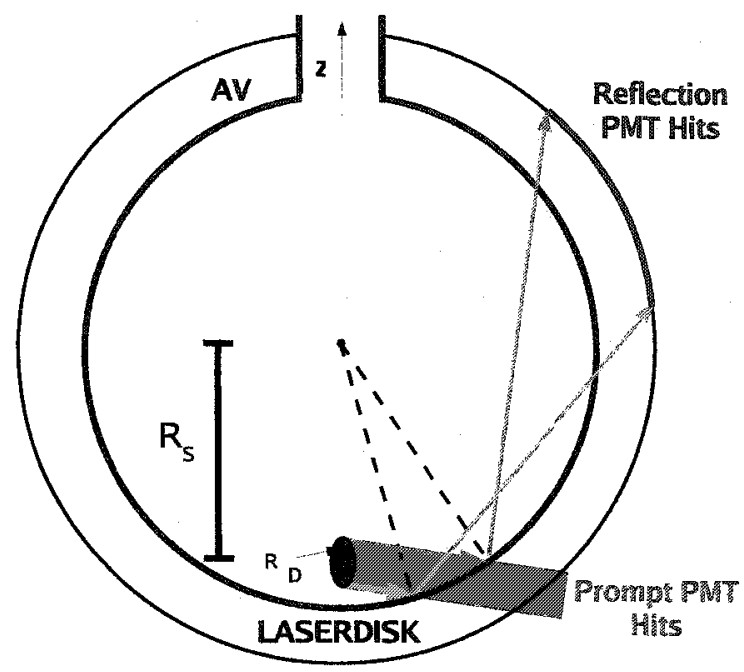

(a) Light paths.

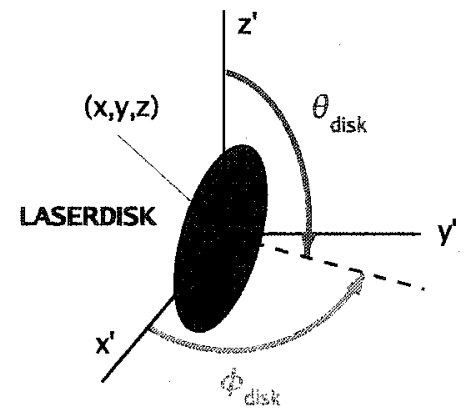

(b) Orientation.

Figure 4.1: Monte Carlo laserdisk source, its light paths, and disk orientation coordinates.

with a laserdisk source with parameters $R_{D}=50 \mathrm{~cm}, R_{S}=550 \mathrm{~cm}, \theta_{\text {disk }}=\pi$ and $\phi_{\text {disk }}=0$. The prompt light hits are seen at the bottom of the PSUP and reflection hits at the top and the equator. For simplicity, the beam and disk orientations are the same $\left(\theta_{\text {beam }}=\theta_{\text {disk }}\right.$ and $\left.\phi_{\text {beam }}=\phi_{\text {disk }}\right)$ so that the photons are leaving the surface at $0^{\circ}$ with respect to the normal of the disk. This simplification is used throughout the remainder of this chapter.

\subsubsection{Laserdisk Time Information}

For a laserdisk source MC run, only a limited number of PMTs are expected to detect light. The direction imposed to the beam, unlike isotropic sources, does not allow a single PMT $n$ to detect both prompt and reflected light. For that reason, the different PMTs need to be tagged: 

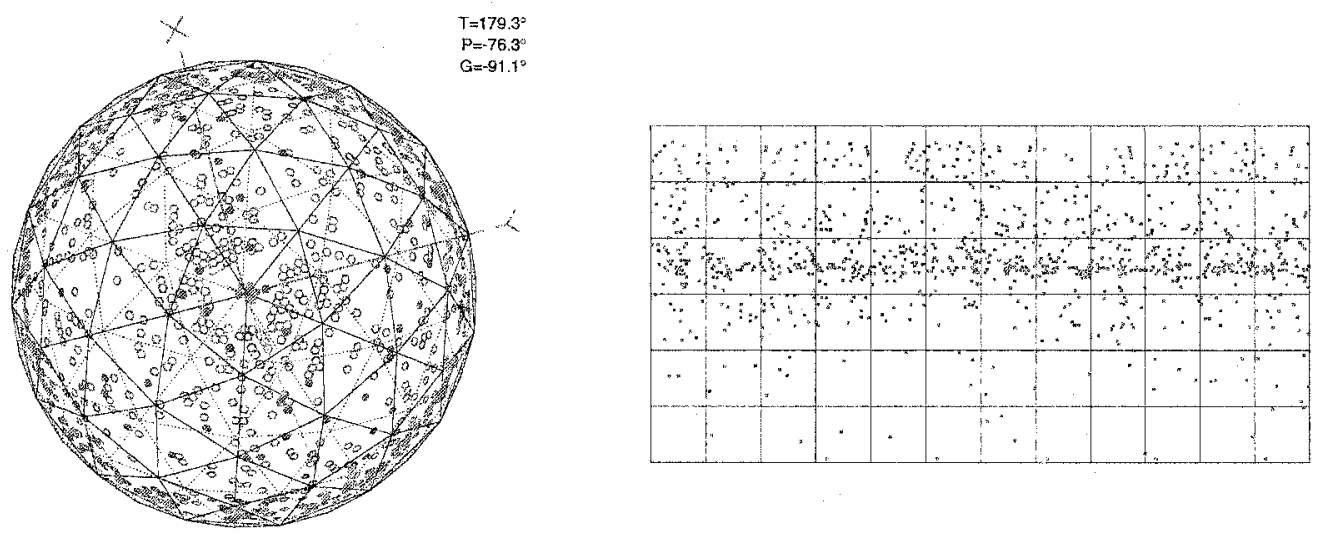

Figure 4.2: View of the PMT hits obtained with a Monte Carlo laserdisk source at $(x, y, z)=(0,0,-550) \mathrm{cm}$ with parameters $R_{D}=50 \mathrm{~cm}, \theta_{\text {disk }}=\pi$, and $\phi_{\text {disk }}=0$. It is shining towards the bottom of the detector. The number of hits per PMT is proportional to the color of the dots, starting from blue (low) to pink (high). The $z$-axis is going into the page in the geodesic sphere view (left). The prompt hits are seen in the center of the figure (pink dots), which corresponds to the bottom of the detector. These hits are at the bottom of the projection view (right). Reflection hits are located at the top and near the equator (blue dots). View this figure in color.

- The index $n=p$ identifies a PMT which detects only prompt light, and

- The index $n=r$ identifies a PMT which detects reflected light.

The laserdisk time information must be obtained by using a PMT pair: a $p$-PMT and a $r$-PMT. A time difference $\delta t$ is calculated from that pair:

$$
\delta t_{\mathrm{disk}}=\mu_{\mathrm{ref}}^{r}-\mu_{\mathrm{prompt}}^{p}
$$

where $\mu_{\text {ref }}^{r}$ is the mean time of the reflection peak for a $r$-PMT, and $\mu_{\text {prompt }}^{p}$ is the mean time of the prompt peak for a $p$-PMT. Note that $\delta t_{\text {disk }}$ is not equivalent to $\Delta t$ since two distinct PMTs are used. 


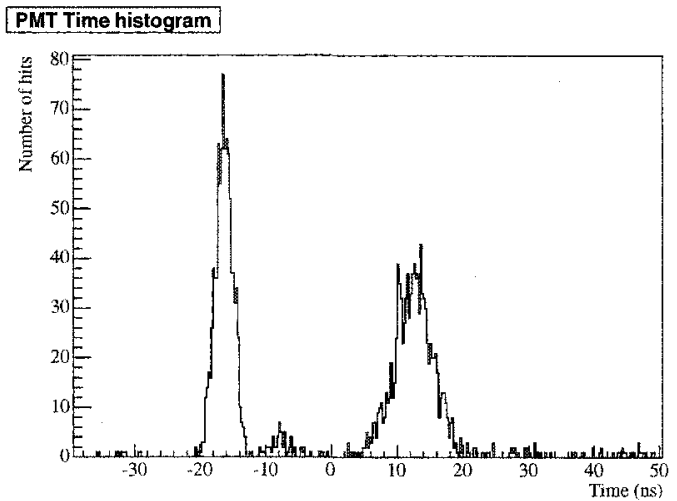

Figure 4.3: Typical time distribution obtained using the laserdisk source. The time distribution is obtained from the example of Figure 4.2. Note that the prompt peak is not centered at $t=0 \mathrm{~ns}$.

Similarly, the sum of all the PMT time histograms also contains a prompt peak and a reflected peak. The prompt peak corresponds to the sum of the all the $p$-PMT prompt peaks, and the reflected peak corresponds to the sum of all the $r$ PMT reflection peaks. The time difference between the reflection and the prompt light peaks is an estimator for $\delta t_{\text {disk }}$ :

$$
\left\langle\delta t_{\mathrm{disk}}\right\rangle=\mu_{\mathrm{ref}}-\mu_{\mathrm{prompt}}
$$

Figure 4.3 shows a typical time distribution obtained using the laserdisk source. It is the sum of all the individual PMT raw time distributions. The left peak is the prompt peak with $\mu_{\text {prompt }} \simeq-16$ ns. The other peaks are reflection peaks.

\subsection{Photon Bomb Source}

The photon bomb is an isotropic point source. Unlike the laserdisk, this source produces light in all directions such that all the PMTs are expected to detect either 

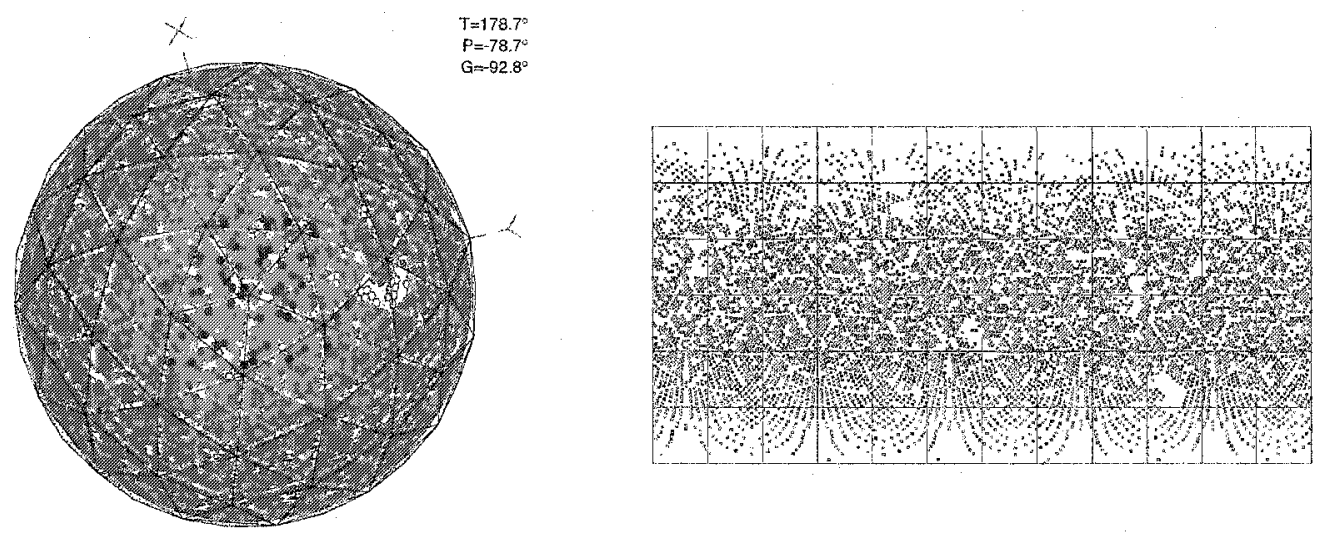

Figure 4.4: View of the PMT hits obtained with a Monte Carlo photon bomb source at $(x, y, z)=(0,0,-550) \mathrm{cm}$. The number of hits per PMT is proportional to the color of the dots, starting from blue (low) to pink (high). The z-axis is going into the page in the geodesic sphere view (left). The prompt hits are seen everywhere in the detector. More hits (pink dots) are seen at the bottom of the detector because the source is closer to that region of the detector. These hits are at the bottom of the projection view (right). View this figure in color.

prompt or reflected light. The photon bomb source parameters are:

- the position $(x, y, z)$, which sets the position radius $R_{S}$ from the origin.

This source does not need any further settings. Figure 4.4 shows an example of the PMT hits for a photon bomb source with $R_{S}=550 \mathrm{~cm}$. The source position and the color scale are the same as in Figure 4.2.

\subsubsection{Photon Bomb Time Information}

The photon bomb gives better information about the prompt light than the laserdisk since a great majority of the PMTs detect the prompt light. The reflected light information is also present but shows up distorted for off-center source positions, as it was shown in the time residual plots of Figure 2.6. For these reasons, only the 


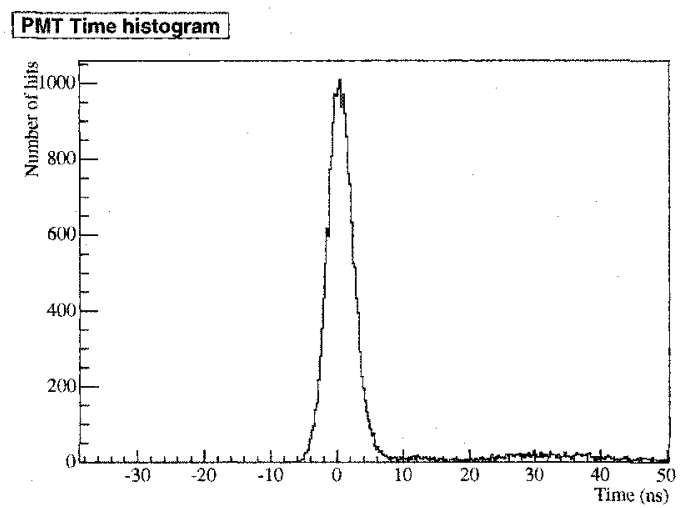

Figure 4.5: Typical time distribution obtained using the photon bomb source. The time distribution is obtained from the example of Figure 4.4. Note that the prompt peak is not exactly centered at $t=0 \mathrm{~ns}$.

prompt light information is used from the photon bomb source.

As before, a PMT $n$ has a prompt peak with mean time $\mu_{\text {prompt }}^{n}$. The photon bomb MC data is used to find the time difference between two PMT prompt peaks:

$$
\begin{gathered}
\delta t_{\text {bomb }}=\mu_{\text {prompt }}^{r}-\mu_{\text {prompt }}^{p} . \\
\delta t_{\text {bomb }}=\mu_{\text {prompt }}^{r}-\mu_{\text {prompt }}^{p} .
\end{gathered}
$$

A conservative estimator for the mean time difference is the full width of the summed distribution prompt peak:

$$
\left\langle\delta t_{\mathrm{bomb}}\right\rangle=2 \sigma_{\mathrm{prompt}}
$$

Figure 4.5 shows a typical time distribution obtained using the photon bomb source. The prompt light peak statistics dominate the time distribution. The reflected light statistics start approximately at $t=10$ ns. 


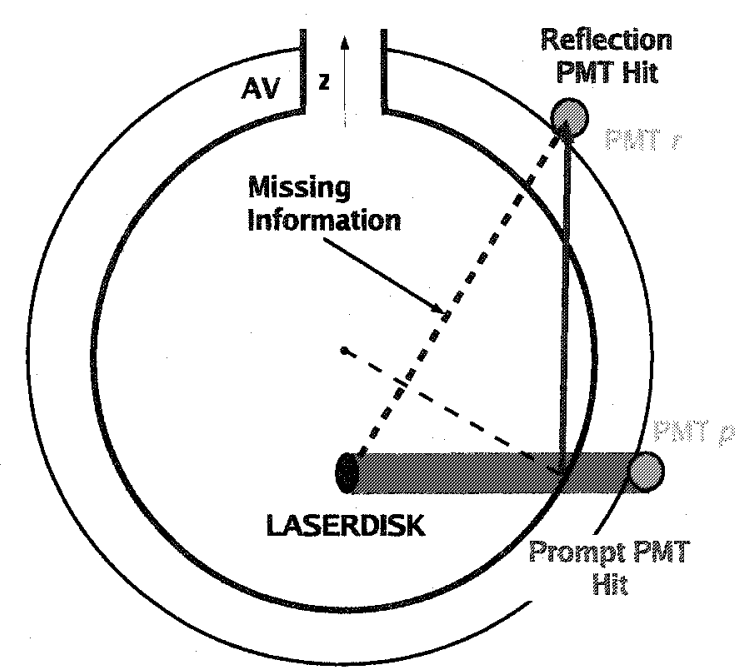

(a) Laserdisk source.

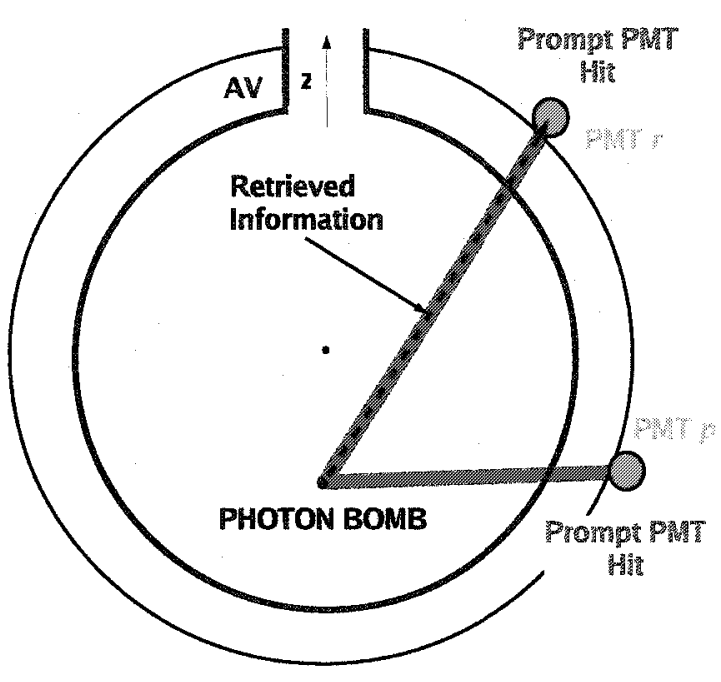

(b) Photon bomb source.

Figure 4.6: Monte Carlo sources complementary information. The laserdisk source does not provide the prompt light path time of detection at a PMT labelled $r$. The photon bomb source is used to recover this information.

\subsection{Combined Timing Information}

Neither the laserdisk nor the photon bomb sources are able to provide a precise measurement of the time difference $\Delta t$, defined in Equation (4.3), needed to compare with the analytic model prediction. Consequently, the laserdisk is used to obtain the reflected light information that the photon bomb cannot precisely provide, and inversely, the photon bomb is used to obtain the prompt light information that the laserdisk provide only partially.

Figure 4.6 shows how the photon bomb source is used to provide complementary time information to the laserdisk source time differences. The missing information from the laserdisk runs is the prompt time $\mu_{\text {prompt }}^{r}$ of a $r$-PMT in Equation (4.5) needed to obtain $\Delta t$. In addition, the $\mu_{\text {prompt }}^{p}$ time is unnecessary. The time differ- 
ence between the reflected and the prompt light detected by a $r$-PMT is found by combining the time differences of Equations (4.5) and (4.7):

$$
\Delta t=\delta t_{\mathrm{disk}}-\delta t_{\mathrm{bomb}}=\mu_{\mathrm{ref}}^{r}-\mu_{\mathrm{prompt}}^{r}
$$

The value $\Delta t$ is a single number for each reflection type per $r$-PMT. This is the time difference to compare with the analytic model $\Delta t_{\text {model }}$.

To enable this time difference comparison using the estimators, Equations (4.6) and (4.8) are combined as in Equation (4.9) to get an estimator of $\Delta t$ :

$$
\begin{aligned}
& \langle\Delta t\rangle=\left\langle\delta t_{\text {disk }}\right\rangle-\left\langle\delta t_{\text {bomb }}\right\rangle \\
& \langle\Delta t\rangle \simeq \frac{1}{N_{T}} \sum_{r}^{N_{T}} \Delta t
\end{aligned}
$$

where $N_{T}$ is the implicit number of $r$-PMTs that detect reflected light in a laserdisk source MC run.

In summary, Equation (4.3) gives the time difference between the reflected and the prompt light detected by a single PMT $n$. To obtain a time difference value similar to $\Delta t_{\text {model }}$ from the MC data, both laserdisk and photon bomb sources are used in a complementary way to obtain Equation (4.9). Equation (4.11) gives the same MC time difference, averaged over the number of $r$-PMTs. Equation (4.9) is more accurate since a PMT-by-PMT comparison is preferred, while Equation (4.11) is a statistical estimator of the time difference. 


\subsection{Comparison with the Analytic Model}

The analytic model of Chapter 3 defines the reflection types (c.f. Table 3.2) and provides a way to calculate the time difference between the AV reflected and the prompt light which strike a given PMT. The optical data selection criterion for PAVRs proposed in Section 3.3 works on a single PMT basis. The MC data time differences $\Delta t$ and $\langle\Delta t\rangle$ must be compared to the results of the analytic model to ensure the latter can be correctly incorporated into the optical data analysis. This comparison is done only with $r$-PMTs, which receive both prompt and reflected light. The results obtained with single PMTs are double-checked with the estimators obtained for all the PMTs. There are two ways of comparing the time differences:

1. Using a single $r$-PMT and Equation (4.9).

2. Using $N_{T} r$-PMTs and Equation (4.11).

The first method gives an MC time difference $\Delta t$ that can be compared directly to the single analytic model value $\Delta t_{\text {model }}$ on a PMT-by-PMT basis. The MC time difference $\Delta t$ has an uncertainty, a consequence of the PMT time distribution width. The uncertainty is calculated with Equation (4.4) using one standard deviation. The second method gives a mean $\mathrm{MC}$ time difference $\langle\Delta t\rangle$ that can be compared with a mean analytic model value, $\left\langle\Delta t_{\text {model }}\right\rangle$. To obtain the latter, many analytic calculations were performed with randomly chosen source positions that lie on the laserdisk surface to get an analytic Gaussian time distribution. 


\subsubsection{Source Configurations}

The photon bomb and the analytic model sources only need a position. However, a slight change in the laserdisk parameters can lead to completely different patterns of PMT hits because of the focused nature of its beam. The radius of the disk $R_{D}$ has been set to $10 \mathrm{~cm}$ based on the results of Appendix B (Section B.1). The beam direction or the disk orientation has to be set. Three configurations are tested to make sure there is no bias in the choice of any particular set of source parameters. The configurations are:

A The position is $(x, y, z)=(0,0,-475) \mathrm{cm}$ for all sources $\left(R_{S}=475 \mathrm{~cm}\right)$. The disk radius is $R_{D}=10 \mathrm{~cm}$, its orientation is $\left(\theta_{\text {disk }}, \phi_{\text {disk }}\right)=\left(\frac{\pi}{2}, 0\right) \mathrm{rad}$.

B The position is $(x, y, z)=(+389,0,-389) \mathrm{cm}$ for all sources $\left(R_{S}=550 \mathrm{~cm}\right)$. The disk radius is $R_{D}=10 \mathrm{~cm}$, its orientation is $\left(\theta_{\text {disk }}, \phi_{\text {disk }}\right)=\left(\frac{3 \pi}{2}, 0\right)$ rad.

C The position is $(x, y, z)=(0,0,-575) \mathrm{cm}$ for all sources $\left(R_{S}=575 \mathrm{~cm}\right)$. The disk radius is $R_{D}=10 \mathrm{~cm}$, its orientation is $\left(\theta_{\text {disk }}, \phi_{\text {disk }}\right)=\left(\frac{\pi}{2}, 0\right) \mathrm{rad}$.

Configurations $\mathrm{A}$ and $\mathrm{C}$ are similar but have different PMTs detecting prompt and reflected light; these configurations are chosen to study HI reflections. For configuration $\mathrm{B}$, the disk shines at the $\mathrm{AV}$ nearly at normal incidence; this configuration is chosen to study LI reflection alone. F reflections are not expected for any of the laserdisk configurations.

Gaussian functions can be fitted to the PMT time histograms with relatively few statistics, so the MC runs are produced generating 500 events with 2500 photons per event. The Rayleigh scattering and media absorption factors are scaled to their minimum values such that these effects do not contribute to the study. The three 


\begin{tabular}{|ccc|}
\hline \hline Setting & Laserdisk & Photon Bomb \\
\hline \hline Source size & $R_{D}=10 \mathrm{~cm}$ & point \\
Photon direction & $\left(\theta_{\text {disk }}, \phi_{\text {disk }}\right)$ & isotropic \\
Wavelength $\lambda$ & $420 \mathrm{~nm}$ & $420 \mathrm{~nm}$ \\
Number of events & 500 & 500 \\
Photons per event & 2500 & 2500 \\
Rayleigh scattering & OFF & OFF \\
Media attenuations & OFF & OFF \\
3D-PMT model & ON & ON \\
\hline \hline
\end{tabular}

Table 4.1: Monte Carlo sources settings.

dimensional PMT reflector model [18] is turned on to get the full detector coverage. Table 4.1 summarizes the fixed settings in SNOMAN for all the MC data presented in this chapter.

The number of events is predefined. The maximum number of hits a PMT can register in a MC run is $N_{\text {max }}^{\text {hit }}=500$ hits assuming the maximum number of hits per event a PMT can have is one. A lower limit is fixed at $N_{\min }^{h i t}=50$ hits so that only tubes with a relatively high number of hits are selected for comparison. The PMTs that belong to the prompt or to the reflected peak can be identified based on the number of hits they have. Filters, defined as $N^{\text {hit }}$ cuts, are applied to isolate the prompt and the reflection peaks from a MC run time distribution. The filters discard the unlikely reflections on the PSUP or the PMT reflectors, and keep most of the LI and HI AV reflections, the ones of interest. The filters applied to the MC data for each configuration are summarized in Table 4.2. The lower limit $N_{\text {min }}^{\text {hit }}$ is higher for configuration $\mathrm{B}$ since the LI reflections are less probable to be seen. The number $N_{T}$ indicates how many good PMT candidates ( $r$-PMTs) are kept after the filters were applied. 


\begin{tabular}{|c|ccc|c|}
\hline \hline Config. & \multicolumn{3}{|c|}{ Filters $\left[N_{\text {min }}^{\text {hit }}, N_{\text {max }}^{\text {hit }}\right]$} & $N_{T}$ \\
\hline \hline & All & Prompt & Reflection & \\
\hline A & {$[0,500]$} & {$[450,500]$} & {$[50,450]$} & 5 \\
B & {$[0,500]$} & {$[450,500]$} & {$[125,450]$} & 3 \\
C & {$[0,500]$} & {$[450,500]$} & {$[50,450]$} & 17 \\
\hline \hline
\end{tabular}

Table 4.2: Laserdisk source configuration $N^{\text {hit }}$ filters. The intervals are expressed in number of hits per PMT in a MC run. The number $N_{T}$ is the number of tubes selected for the comparison based on the prompt and reflection filters.

All the PMT hits obtained using the three laserdisk source configurations are shown in Figure 4.7. The prompt and reflection PMT hits are both needed for the MC time differences calculations. The $p$ - and $r$-PMTs selected after the filters given in Table 4.2 were applied are explicitly shown in Figure 4.8. The PMT-by-PMT time difference comparison is made by extracting the time information of the $N_{T}$ PMT channels for each configuration.

\subsubsection{Results}

The laserdisk time distributions were filtered to isolate the prompt peak and the reflection peaks to be fitted separately for greater precision. The photon bomb time distributions were not filtered since they are used to extract the prompt peak information only. The mean time values, $\mu^{n}$, extracted from single PMT time distributions, and $\mu$, from the fit to the summed distributions, are used in Equations (4.9) and (4.10), respectively, to calculate the MC time differences. Tables 4.3, 4.4, and 4.5 give the time comparison results for the $N_{T}$ PMTs of configurations A, B, and C, respectively. The PMT numbers $n$ are given with their $N^{\text {hit }}$ in the corresponding 


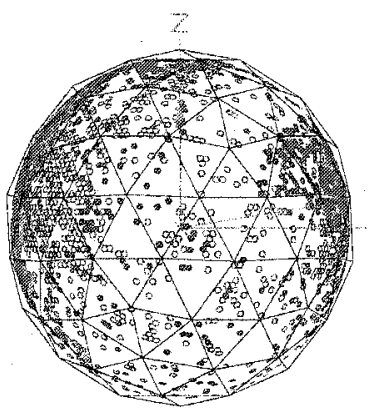

(a) Config. A.

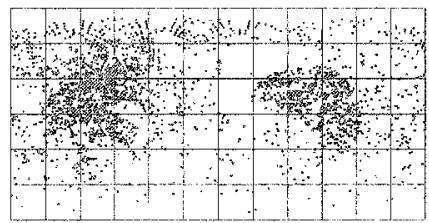

(d) Config. A.

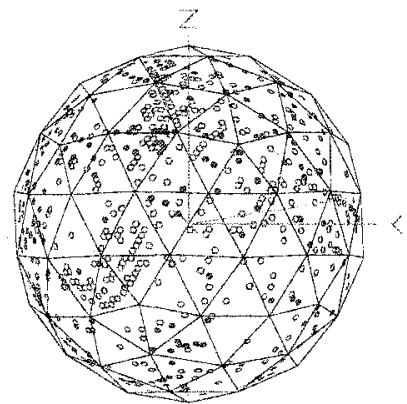

(b) Config. B.

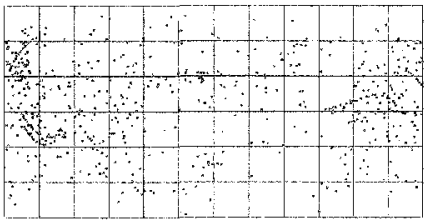

(e) Config. B.

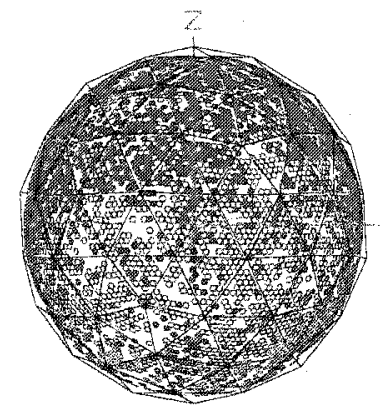

(c) Config. C.

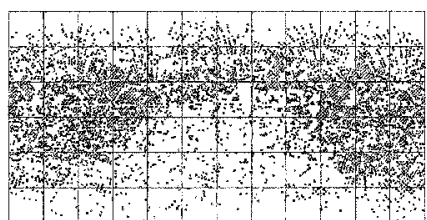

(f) Config. C.

Figure 4.7: Sphere and rectangular projection views of all the PMT hits of each laserdisk configuration. All the hits are shown: the filter is 'All'. The beam is always pointing in the $x$ direction $\left(\phi_{\text {disk }}=0\right)$. The $x$-axis points out of the page for the sphere view, all $\phi=0$ PMTs lie on the vertical central line for the rectangular projection view. View this figure in color.

MC run. The reflection type and the incident angle on the AV as calculated by the model, $\alpha_{\text {model }}$, are also provided. Table 4.6 gives the time comparison results using the time difference estimators. Section B.2 of Appendix B contains the details of the fit results for the PMT time distributions taken from both the laserdisk and photon bomb MC runs in each configuration.

\subsection{Discussion}

The bulk of the time differences compared in Tables 4.3, 4.4, 4.5, and 4.6 agree within the uncertainties. Each configuration is discussed in more details below. 


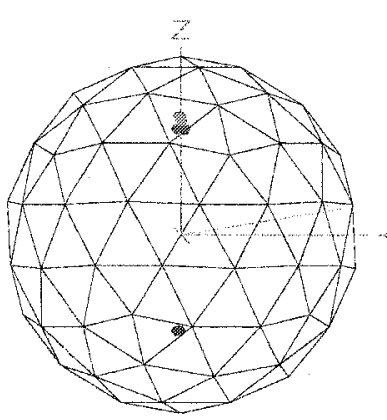

(a) Filtered config. A.

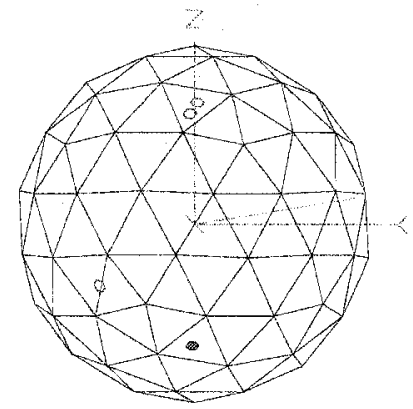

(b) Filtered config. B.

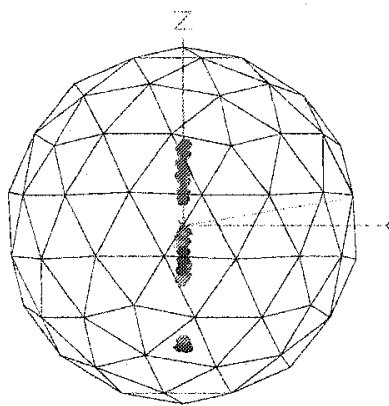

(c) Filtered config. C.

Figure 4.8: Sphere view of the PMT filtered hits of each laserdisk configuration. The prompt and reflection hits are shown: the filter is 'Prompt' + 'Reflection'. It is the same as Figure 4.7 without the low $N^{\text {hit }}$ PMTs. The sphere view shows the prompt PMT hits in front in the lower hemisphere. PMT hits near the equator and at the top of the detector are AV reflected light hits. View this figure in color.

\subsubsection{Time Differences}

For configuration A, the laserdisk source is used to study HI reflections at a relatively large source position radius $R_{S}=475 \mathrm{~cm}$. The $\Delta t$ values of Table 4.3 are consistent for the PMTs selected. However, the values given by the MC is always larger by approximately $2 \mathrm{~ns}$. This is characteristic of the large distance between the $p$-PMTs and the $r$-PMTs. In this case, the $\delta t_{\text {bomb }}$ time difference obtained with the photon bomb corresponds to approximately $\sigma_{\text {prompt }}$ causing $\delta t_{\text {bomb }}$ to be underestimated. The estimators for $\Delta t(\langle\Delta t\rangle)$ found in Table 4.6 are nevertheless consistent. In this case, the difference between the MC and the analytic value is smaller since the width of the bomb prompt peak $\left\langle\delta t_{\text {bomb }}\right\rangle=2 \sigma_{\text {prompt }}$ is larger than $\sigma_{\text {prompt }}$. Hence, the model is consistent with the MC.

For configuration B, the laserdisk source is used to study LI reflections at a high source position radius $R_{S}=550 \mathrm{~cm}$. Most of the $\Delta t$ values of Table 4.4 are not 


\begin{tabular}{|cc|cc|cc|}
\hline \hline \multicolumn{5}{|c|}{ Configuration A } \\
\hline PMT & $N^{\text {hit }}$ & $\mathrm{R}$ & $\alpha_{\text {model }}$ & $\Delta t$ & $\Delta t_{\text {model }}$ \\
\hline \hline 8441 & 286 & HI & 52.3 & $12.3 \pm 2.2$ & 9.8 \\
8445 & 91 & HI & 52.2 & $12.2 \pm 1.9$ & 9.6 \\
8446 & 104 & HI & 52.1 & $12.3 \pm 2.6$ & 9.4 \\
8925 & 189 & HI & 52.1 & $13.2 \pm 2.1$ & 10.9 \\
8927 & 306 & HI & 52.3 & $12.4 \pm 2.1$ & 10.6 \\
\hline \hline
\end{tabular}

Table 4.3: Time differences comparison for the $N_{T}=5$ selected PMTs of configuration $A$. All times are in $n$ s and angles in degrees. $R$ is the reflection type tested. $\alpha_{\text {model }}$ is the incident angle found by the model.

\begin{tabular}{|cc|cc|cc|}
\hline \hline \multicolumn{5}{|c|}{ Configuration B } \\
\hline PMT & $N^{\text {hit }}$ & $\mathrm{R}$ & $\alpha_{\text {model }}$ & $\Delta t$ & $\Delta t_{\text {model }}$ \\
\hline \hline 4388 & 214 & LI & 0.77 & $7.5 \pm 2.1$ & 4.6 \\
5294 & 153 & - & - & $26.8 \pm 1.8$ & - \\
5463 & 135 & LI & 3.08 & $30.2 \pm 2.1$ & 4.6 \\
\hline
\end{tabular}

Table 4.4: Time differences comparison for the $N_{T}=3$ selected PMTs of configuration $B$. All times are in $n s$ and angles in degrees. $R$ is the reflection type tested. $\alpha_{\text {model }}$ is the incident angle found by the model.

consistent because of a lack of PMTs selected. There is only one good PMT available for the comparison: $n=4388$. The difference between the MC and the model value can be explained using the same argument as for configuration $\mathrm{A}$ since the $p$-PMTs and the $r$-PMTs are separated by roughly $2 R_{P S U P}$. The other PMTs have their reflection peaks up to $30 \mathrm{~ns}$ after the prompt, which cannot be explained by the model. There must be other reflections happening for these PMTs, which are out of reach of the prompt peak if the time difference is 30 ns. For PMT $n=5463$, the model was able to find one LI solution, but there is no peak at the same time in 


\begin{tabular}{|c|c|c|c|c|c|}
\hline \multicolumn{6}{|c|}{ Configuration $\mathrm{C}$} \\
\hline PMT & $N^{h i t}$ & $\mathrm{R}$ & $\alpha_{\text {model }}$ & $\Delta t$ & $\Delta t_{\text {model }}$ \\
\hline \multirow[t]{2}{*}{8856} & 418 & $\mathrm{HI}(1)$ & 54.5 & & 1.4 \\
\hline & & $\mathrm{HI}(2)$ & 71.3 & $-0.8 \pm 2.7$ & 2.0 \\
\hline \multirow[t]{2}{*}{8857} & 370 & $\mathrm{HI}(1)$ & 61.7 & & 1.2 \\
\hline & & $\mathrm{HI}(2)$ & 73.0 & $-0.6 \pm 2.7$ & 1.4 \\
\hline \multirow[t]{2}{*}{8859} & 322 & $\mathrm{HI}(1)$ & 64.6 & & 1.1 \\
\hline & & $\mathrm{HI}(2)$ & 73.4 & $0.0 \pm 2.8$ & 1.2 \\
\hline \multirow[t]{2}{*}{8862} & 445 & $\mathrm{HI}(1)$ & 69.7 & & 0.93 \\
\hline & & $\mathrm{HI}(2)$ & 72.7 & $-1.3 \pm 2.7$ & 0.95 \\
\hline 8991 & 107 & $\mathrm{HI}(2)$ & 72.7 & $1.1 \pm 2.8$ & 1.5 \\
\hline 8996 & 294 & $\mathrm{HI}(2)$ & 73.0 & $0.6 \pm 2.7$ & 1.4 \\
\hline \multirow[t]{2}{*}{8997} & 427 & $\mathrm{HI}(1)$ & 62.9 & & 1.1 \\
\hline & & $\mathrm{HI}(2)$ & 73.2 & $-0.4 \pm 2.7$ & 1.3 \\
\hline \multirow[t]{2}{*}{9001} & 356 & $\mathrm{HI}(1)$ & 64.6 & & 1.1 \\
\hline & & $\mathrm{HI}(2)$ & 73.2 & $0.3 \pm 2.8$ & 1.3 \\
\hline \multirow[t]{2}{*}{9002} & 447 & $\mathrm{HI}(1)$ & 63.1 & & 1.1 \\
\hline & & $\mathrm{HI}(2)$ & 73.2 & $-1.2 \pm 2.6$ & 1.3 \\
\hline 9092 & 122 & $\mathrm{HI}(1)$ & 65.1 & $4.9 \pm 2.8$ & 5.0 \\
\hline 9093 & 87 & $\mathrm{HI}(1)$ & 65.6 & $6.1 \pm 2.8$ & 4.8 \\
\hline 9094 & 117 & $\mathrm{HI}(1)$ & 65.1 & $5.2 \pm 2.8$ & 5.0 \\
\hline 9096 & 77 & $\mathrm{HI}(1)$ & 66.9 & $4.5 \pm 2.8$ & 4.0 \\
\hline 9602 & 127 & $\mathrm{HI}(1)$ & 67.7 & $3.9 \pm 2.6$ & 3.6 \\
\hline 9603 & 132 & $\mathrm{HI}(1)$ & 69.4 & $4.1 \pm 2.8$ & 2.8 \\
\hline 9606 & 143 & $\mathrm{HI}(1)$ & 68.1 & $3.4 \pm 2.9$ & 3.4 \\
\hline 9608 & 131 & $\mathrm{HI}(1)$ & 69.0 & $2.7 \pm 2.7$ & 3.0 \\
\hline
\end{tabular}

Table 4.5: Time differences comparison for the $N_{T}=17$ selected PMTs of configuration $C$. All times are in $n s$ and angle in degrees. $R$ is the reflection type tested. $\alpha_{\text {model }}$ is the incident angle found by the model. Labels are used in cases where more than one HI reflection is expected. HI(1) reflections have lower $\alpha_{\text {model }}$ than HI(2)s. 


\begin{tabular}{|c|c|cccc|}
\hline \hline Config. & $\mathrm{R}$ & $\left\langle\delta t_{\text {disk }}\right\rangle$ & $\left\langle\delta t_{\text {bomb }}\right\rangle$ & $\langle\Delta t\rangle$ & $\langle\Delta t\rangle_{\text {model }}$ \\
\hline $\mathrm{A}$ & $\mathrm{HI}$ & $14.5 \pm 2.3$ & $3.85 \pm 0.02$ & $10.6 \pm 2.3$ & $9.8 \pm 0.6$ \\
\hline $\mathrm{B}$ & $\mathrm{LI}$ & $9.3 \pm 2.2$ & $3.95 \pm 0.02$ & $5.3 \pm 2.2$ & $4.6 \pm 0.7$ \\
\hline $\mathrm{C}$ & $\mathrm{HI}$ & $3.6 \pm 3.6$ & $3.93 \pm 0.02$ & $-0.3 \pm 3.6$ & $2.1 \pm 1.3$ \\
\hline \hline
\end{tabular}

Table 4.6: Time difference estimators comparison for the $N_{T}$ PMTs of each configuration. All times are in $n s . R$ is the reflection type tested. See Appendix $B$ for the fit results of all the separate light peaks.

the MC data. This can be explained by the actual alignment of the PMT-reflector assembly on the PSUP. The $\langle\Delta t\rangle$ comparison values are consistent, but depends on a single PMT $n=4388$.

For configuration $\mathrm{C}$, the laserdisk source is used to study multiple HI reflections at a high source position radius $R_{S}=575 \mathrm{~cm}$. The $\Delta t$ values of Table 4.5 are consistent within the errors for the PMTs selected. The errors are larger in this case since both the prompt and reflection peaks are wider, a consequence of the larger incident angle at that source position. The MC time difference is only found once even if the analytic model finds two HI reflections. Since the HI reflections have approximately the same $\Delta t_{\text {model }}$, the $\mathrm{MC}$ reflection peak is the addition of the two reflections. This happens for PMTs with high $N^{\text {hit }}$, comparable to the maximum $N_{\max }^{\text {hit }}=500$. The reflections cannot be decoupled in time in the MC time distributions. The $\langle\Delta t\rangle$ estimators are also consistent. In this case, the estimator $\left\langle\delta t_{\text {bomb }}\right\rangle$ is too large since the distance between the $p$-PMTs and the $r$-PMTs is small. It is the opposite effect of what was found for configuration A. 


\subsubsection{Reflection Causes and Types}

The PMT hit maps of Figures 4.7 and 4.8 provide evidence that the PAVRs are the most important reflections to consider at high radius. It is explainable by the fact that the source is closer to the AV than any other detector component.

The filters were applied to discard other possible reflections. There are numerous PMTs detecting these reflections that have a low number of hits in a run. The reflections are most likely coming from the PMT reflectors since the 3D-PMT model was used in the simulations. More details are given in Section B.3.2 of Appendix B.

The results provided by the configuration $\mathrm{C}$ are very important. The HI reflections have a large angle of incidence on the AV, $\alpha_{\text {model }}>45^{\circ}$, giving a large probability of reflection. Both the MC and the model agree for the probability of occurrence of these reflections based on the number of hits $N^{\text {hit }}$ for the MC, and on the two different HI reflections found for most PMTs by the model.

\subsubsection{Summary}

The laserdisk source is used to focus photon beams on specific zones of the detector to isolate the effect of the $\mathrm{AV}$ reflections. The $\mathrm{MC}$ data provides time differences, between the reflected and the prompt light detected by a PMT, in agreement with the values predicted by the AV reflection analytic model. In addition, the MC data verifies that the primary reflection effect in terms of reflection probability and short time differences for high radius source positions is due to the PAVRs. Chapter 5 explains how to implement the model in the default optics code, since the MC and the analytic model data are consistent. The results of these changes to the data processing and the effects on the optical constants are presented in Chapter 6 . 


\section{Chapter 5}

\section{Implementation of the New Code}

The reflected light information presented in Section 3.3, given by QOCAReflect and QOptics, is consistent with the reflected light that can be tracked by SNOMAN. The AV reflection model must be added to the optical data processing such that the PAVRs are identified and the PMTs that might detect them are flagged at the data organization step of the analysis. This chapter explains how the new code is inserted and used within the optical data processing chain.

\subsection{Adding the New Code}

It was shown in Chapter 3 that the QOCAReflect and QOptics classes can be used outside of a data processing context because they use the SNOMAN detector geometry. The classes were used to simulate or extract the prompt and reflected light path information from a given source position to all the PMT positions of the geometry. Thus, the QOCAReflect code can be inserted between two processing steps, as it is intended not to change significantly the way the data is currently processed. 


\subsubsection{Calculations}

A QOCAReflect object can be created and used to perform a series of calculations in a block without interfering with the other QOCA calculations. The primary operations of QOCAReflect are to:

- use the analytic model to find the AV reflection solution(s) given a source position and a PMT,

- use QOptics to calculate the reflected light paths and their associated time delays, and,

- provide the limits on $\cos \theta_{p s}$ as a cut to be applied on the data.

QOCAReflect does not flag the PMTs. It only provides the information needed by QOptics and QPath to flag the PMTs in a general way.

\subsubsection{Speed or Precision}

There are two simple ways to implement the new QOCAReflect code into the current optical data processing:

1. Produce the $\cos \theta_{p s}$ cut limits before the analysis and write them to a data table. The cut limits are loaded during the analysis from the data table.

2. Find the necessary $\cos \theta_{p s}$ cut limits at run time.

Method 1 is faster but introduces an additional systematic error since the table data may not represent the same positions as those taken in a specific scan. A table produced with many source positions having the same radius gives good estimators 
of the real cut limits, but also allows for statistical fluctuations. Method 2 is slower because it has to be calculated for each high radius position at run time. A scan containing eight to ten high radius runs per wavelength would take approximately ten additional minutes to process with the new QOCAReflect code. This method is longer. However it gives the exact information for each high radius source position in a scan. Hence, method 2 is preferred for the analysis of the following chapters.

\subsection{Class Parameters}

It was mentioned in Section 3.3 that the QOCAReflect code needs two parameters to do its work: the source position radius $R_{S}$ and the reflection time window acceptance $t_{w}$. This section explains how these parameters are used and determined.

\subsubsection{Source Position Radius}

The $R_{S}$ parameter is supplied to the code by the actual positions of the source in a run. It is used as an input to the AV reflection analytic model to verify if there are potential reflections for each PMT of the array. The total number of reflections $N_{r}$, the number of reflections per PMT $k$, and the time differences $\Delta t^{k}$ are all source position or run dependent.

\subsubsection{Time Window Acceptance}

Before the new PAVR code can be used in the optical data processing module, the time window parameter $t_{w}$ must be conveniently set. Recall this parameter is the minimum allowed time difference between the reflected and the prompt light. In 
principle, this time window should be as small as possible given the condition of a run. The optimal choice of the time window $t_{w}$ is found using $\mathrm{MC}$ data. The following explains how it was set for the analyses of Chapter 6 .

The time residual plots (c.f. Chapter 2) show that the prompt light peak dominates the time spectrum for each source position. For each run, the prompt peak obtained is centered at $t=0 \mathrm{~ns}$. The optical analysis prompt time window is also centered at $t=0 \mathrm{~ns}$ and selects the prompt light on both sides of the peak, for each PMT channel. The current prompt peak time window is $8 \mathrm{~ns}$ wide ( 4 ns half-width). It is set to include approximately $2 \sigma_{\text {prompt }}^{n}$, or $95.4 \%$, of the direct light on each side of the peak, assuming a Gaussianly distributed peak.

The reflection time window $t_{w}$ must remove most of the reflections in the prompt peak of a single PMT $n$ in the $t= \pm 2 \sigma_{\text {prompt }}^{n}$ time interval. The time difference $\Delta t$ between the reflected and the prompt light is the difference between both peak mean times as defined in Equation (4.3). The reflection time window is applicable from the prompt peak mean time $\mu_{\text {prompt }}^{n}$ to the reflection peak mean time $\mu_{\text {ref }}^{n}$. Thus, the time difference between the reflected and the prompt light reaching a PMT $n$ must always be larger than the time window acceptance parameter $t_{w}$ :

$$
\Delta t=\mu_{\mathrm{ref}}^{n}-\mu_{\mathrm{prompt}}^{n}>t_{w}
$$

It is necessary for the reflection time window to go further than the end of the prompt peak since the reflected light is also Gaussianly distributed, characterized by a mean time and a standard deviation (c.f. Chapter 4 ). On the other hand, $t_{w}$ cannot be set naively to $2 \sigma_{\text {prompt }}^{n}$, at the edge of the prompt peak acceptance, since that would let $50 \%$ of the reflected light enter the prompt peak at $\Delta t=t_{w}$. It must 
be set further away such that the tails of both peaks do not significantly overlap:

$$
2 \sigma_{\text {prompt }}^{n}<\Delta t-2 \sigma_{\text {ref }}^{n}
$$

which means

$$
2 \sigma_{\text {prompt }}^{n}+2 \sigma_{\text {ref }}^{n}>t_{w}
$$

This inequality already suggests what value to use for $t_{w}$.

From the discussion of Section 4.6, there is no right way to find the prompt peak width $\sigma_{\text {prompt }}^{n}$. The choice of $\left\langle\delta t_{\text {bomb }}\right\rangle$ (or $\sigma_{\text {prompt }}^{n}$ ) was most of the time the cause of the discrepancy between the analytic time difference and the combined MC time difference. To account for this uncertainty, the width of the summed distribution $\sigma_{\text {prompt }}=2$ ns can be used instead of $\sigma_{\text {prompt }}^{n}$, assuming $\sigma_{\text {prompt }}<\sigma_{\text {prompt }}^{n}$.

The laserdisk PMT time distributions obtained for the MC checks of Chapter 4 provide a good estimate of the reflection peak distribution width, $\sigma_{\text {ref }}^{n}$. The width of the reflection peak is larger for higher source radii. The MC run with the highest radius had $R_{S}=575 \mathrm{~cm}$ (configuration $\mathrm{C}$ ). Hence, the reflection peak width found from that MC run can be used as an upper limit. This upper limit corresponds to $\sigma_{\text {ref }}^{n} \simeq 2$ ns. Therefore, the time window parameter is chosen to be:

$$
t_{w}=2 \sigma_{\mathrm{prompt}}+2 \sigma_{\mathrm{ref}}^{n} \simeq 4 \mathrm{~ns}+4 \mathrm{~ns}=8 \mathrm{~ns} .
$$

Figure 5.1 shows both peaks and the time window ranges of application. The $t_{w}$ parameter determines the full width of the reflection time window. The amount of reflected light entering the prompt peak is approximately $2.3 \%$ of the entire reflected 


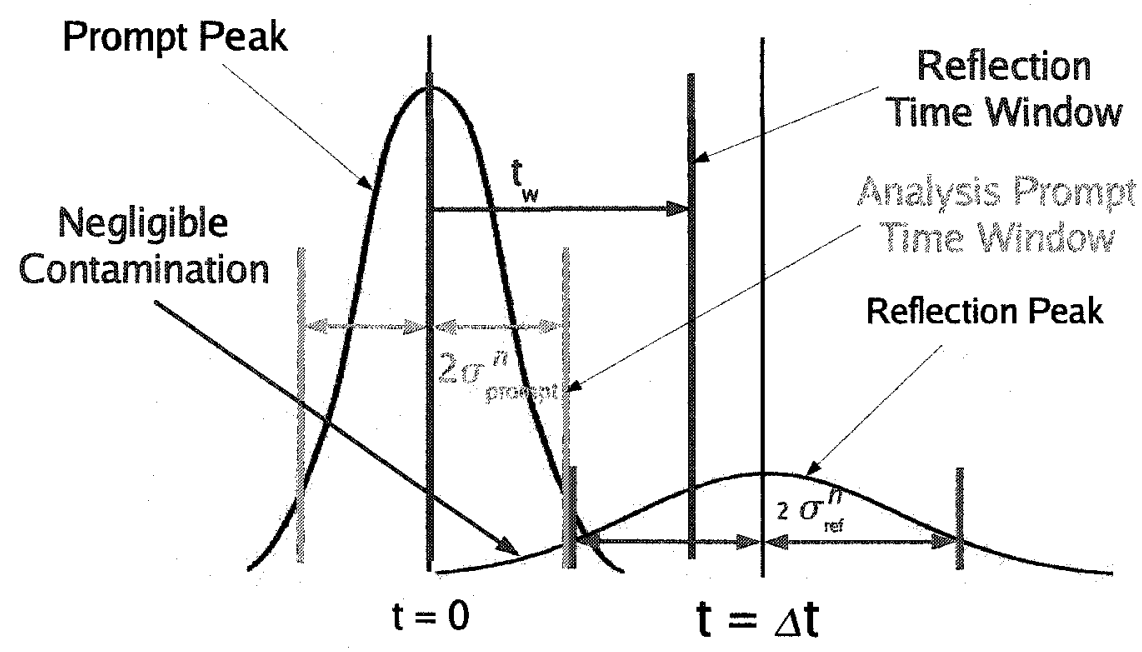

Figure 5.1: Time windows acting on the PMT time data. The analysis prompt time window is centered at $t=0 \mathrm{~ns}$; it defines the prompt light peak mean time. The reflection time window starts from that prompt light peak mean time and goes $t_{w} n s$ later. The reflection light peak mean time comes $\Delta t$ after the prompt peak. Even if the reflection peak of this example is not rejected by the cut, there is still a negligible amount of contamination entering the prompt peak. Note that the reflection peak size has been exaggerated. View this figure in color.

light amount, which is negligible for the analysis. The new PAVR selection criterion rejects all reflections within the range $\Delta t=\left[0, t_{w}\right]$ ns. If the inequality of Equation (5.1) is not fulfilled, the usual flag $f_{\text {pavr }}$ is raised and the PMT contributes to the number of bad PMTs $N_{\text {bad }}$ not used in the optical model fit.

\subsubsection{Summary}

As proposed in Section 2.3, the current constant cut $\cos \theta_{p s}<0.1$ applied on PMTs can be replaced by a radius dependent cut based on the information given by the AV reflection analytic model. From the data of Table 3.3 , the $\cos \theta_{p s}$ limits are dependent on the source position radius. Therefore, the new PAVR PMT selection must be 


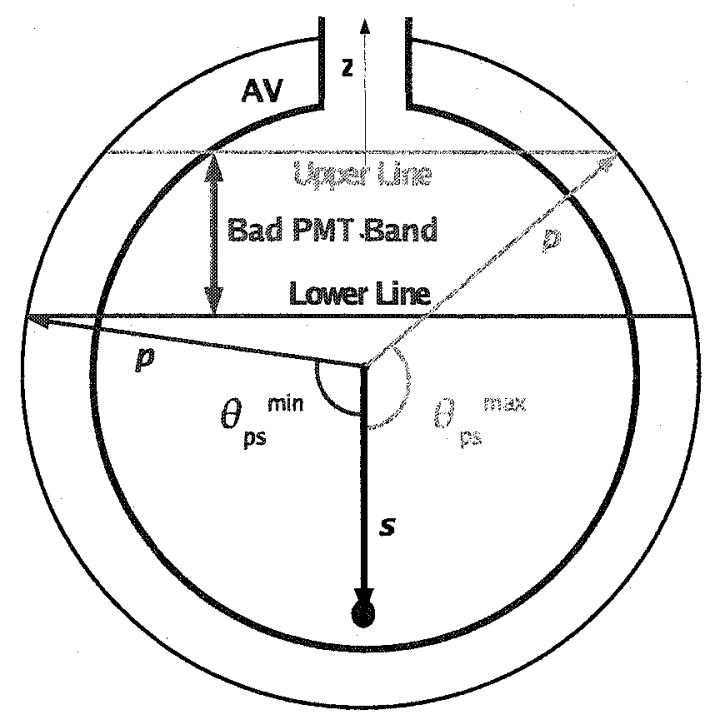

(a) Schematic view of the cut.

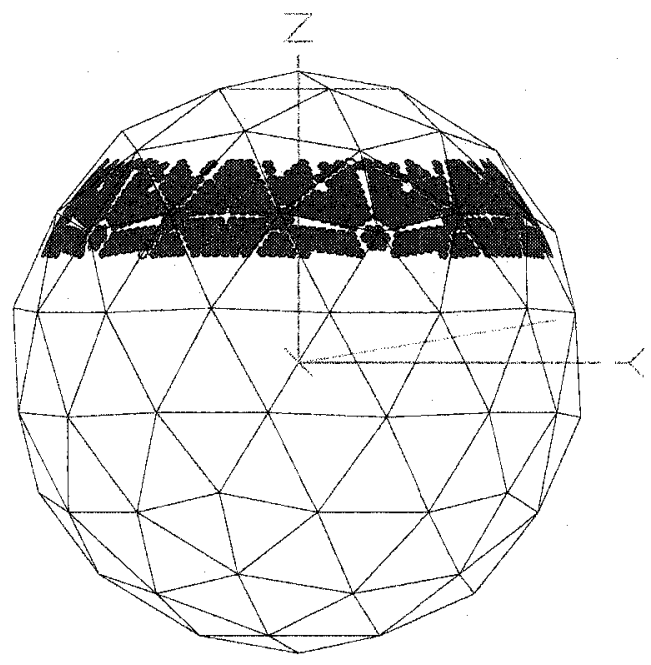

(b) Sphere view of the cut.

Figure 5.2: Simplistic view of the modified cut due to prompt AV reflections. The lines which set the limits to the band cut are both source radius and time window dependent. The cut limits must be found for each position. The sphere view shows an example of that cut for $(x, y, x)=(0,0,-500) \mathrm{cm}$ with $t_{w}=8 \mathrm{~ns}$. View this figure in color.

made independently for each source position. The source radius $R_{S}$ parameter affects the number of possible reflections $N_{r}$ and the time window $t_{w}$ parameter affects the number $N_{\text {bad }}$ of contaminated tubes. As an example, Figure 5.2 shows schematically the $\cos \theta_{p s}$ cut for $R_{S}=500 \mathrm{~cm}$ and $t_{w}=8 \mathrm{~ns}$. The PMTs rejected in Figure 5.2(b) should be compared with the $85^{\circ}$ rejection of Figure 2.4 p. 26 . It is clear that the new PAVR selection criterion is more efficient and reflects a better modelling of the AV reflections since $N_{\text {bad }}$ varies with the source position radius. 


\section{Chapter 6}

\section{Results of the Enhanced}

\section{Processing Method}

The addition of the AV reflection model to the optical data analysis allows the previously unused high radius runs $\left(R_{S}>500 \mathrm{~cm}\right)$ to be analyzed for the following reasons: the nature of the AV reflections affecting the occupancy of many PMTs in these runs is now known, and a radius dependent selection criterion is used to flag the PMTs detecting PAVRs. Thus, the enhanced processing method, defined as the analysis of the whole data set of a $\mathrm{D}_{2} \mathrm{O}$ scan using the new PAVR selection criterion, has been used to reprocess the data of the October 2003 scan. This scan was the most recent at the time the enhanced method was ready to be tested. The acronym for the scan is 'oct03'. It corresponds to the second $\mathrm{D}_{2} \mathrm{O}$ phase, the transition phase between the salt phase and the NCD phase of the SNO experiment. The oct03 optical constants were first obtained by Ranpal S. Dosanjh at Carleton and are consistent with the previous scans of both the $\mathrm{D}_{2} \mathrm{O}$ and salt phases [19]. 
This chapter provides a comparison of some of the optical parameters obtained after fitting, using the occupancy ratio method, the cleaned data obtained with both the enhanced (new) and the standard (default) processing methods. Consequently, the impact of the new PAVR selection criterion is depicted by looking at the optical parameters, namely the $\mathrm{D}_{2} \mathrm{O}$ and $\mathrm{H}_{2} \mathrm{O}$ attenuations, and the PMT angular response. Then the uncertainties of these optical parameters are propagated to the energy scale uncertainty used in neutrino event reconstruction.

\subsection{Changes in the Statistics}

This section presents a comparison of the statistics available after organizing the data using the standard and the enhanced processing methods. The resulting changes from switching from one processing method to another are observed by going through the data trees, run by run, and by looking at the flags assigned by both methods to each PMT of each run. When a PMT is flagged, the flag takes a value of 1 . Otherwise the PMT is fine and the flag remains 0 , the default value. The relevant flags for high radius and PAVR cuts are $f_{\text {bad }}$ and $f_{\text {pavr }}$. Since the fit method uses the occupancy ratios, the flag for central runs, $f_{\text {cbad }}$, is also studied. Table 6.1 contains a summary of these flags allowed values and their meanings. When $f_{\text {pavr }}$ is enabled, $f_{\text {bad }}$ is automatically enabled, but the reverse is not true. The flag $f_{\text {parr }}$ cannot be enabled for central runs since it can only be assigned to high radius run PMTs. The column labelled 'Fit' in Table 6.1 tells if the PMT is used in the fit.

The changes in the statistics are verified through the total number of valid PMTs in the scan asking for the condition $f_{\mathrm{bad}}=f_{\text {cbad }}=0$ to be fulfilled. Since the number 


\begin{tabular}{|ccccc|}
\hline \hline$f_{\text {bad }}$ & $f_{\text {cbad }}$ & $f_{\text {pavr }}$ & Meaning & Fit \\
\hline \hline 0 & 0 & 0 & Good PMT & Yes \\
\hline 0 & 1 & 0 & Central run PMT flagged & No \\
\hline 1 & 0 & 0 & Off-center run PMT flagged & No \\
\hline 1 & 1 & 0 & Both run PMTs flagged & No \\
\hline 1 & 0 & 1 & PMT flagged for PAVRs & No \\
\hline \hline
\end{tabular}

Table 6.1: QOptics flag details and meanings, repeated from Table 2.1. The last column indicates if the PMT contributes to the occupancy ratio fit.

of PMTs analyzed is variable from one scan to another, it is better to look at the statistics available as a function of a PMT observable for a given scan. The incident angle of the incoming light on the PMTs, $\theta_{P M T}$, is the observable chosen to organize the PMTs according to the flags that they carry. In the following, the number of PMTs per bin of incident angle is plotted for each flag for both the enhanced and the standard processing methods. It is done first for particular high radius runs at $\lambda=500 \mathrm{~nm}$, and then for the entire scan at $\lambda=420 \mathrm{~nm}$ (all the runs at that wavelength).

\subsubsection{Statistics of High Radius Runs}

The effect of using the new PAVR cut to select the data can be isolated by looking at the statistics of high radius runs. Two runs with $R_{S}>450 \mathrm{~cm}$ were selected. Run 34865 has $R_{S} \simeq 500 \mathrm{~cm}$ and run 34964 has $R_{S} \simeq 550 \mathrm{~cm}$. Figures 6.1 and 6.2 contain the number of PMTs per incident angle bin ( $1^{\circ}$ wide) for two selected flag combinations of Table 6.1. In each figure, the legend identifies the statistics obtained from both processing methods. The difference is defined as $\Delta \equiv \mid$ enhanced - standard|. 


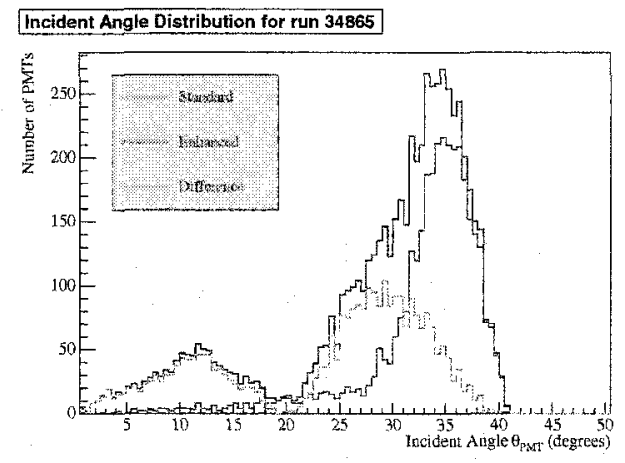

(a) $f_{\text {bad }}=0, f_{\text {cbad }}=0$.

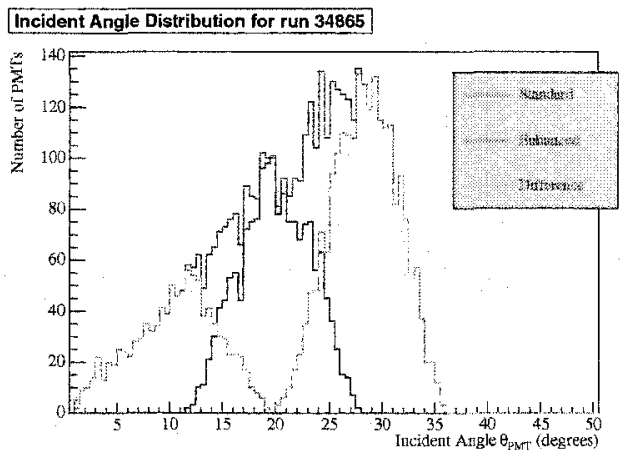

(b) $f_{\text {bad }}=1, f_{\text {pavr }}=1$.

Figure 6.1: Effect of applying the new PAVR cut on the statistics of run 34865 as a function of the incident angle $\theta_{P M T}$. Run 34865 has $R_{S}=500 \mathrm{~cm}$ in the October 2003 scan at $\lambda=500 \mathrm{~nm}$. The difference curve in each case corresponds to the change in statistics. Figure a) shows the total number of PMTs used in that run. View these plots in color.

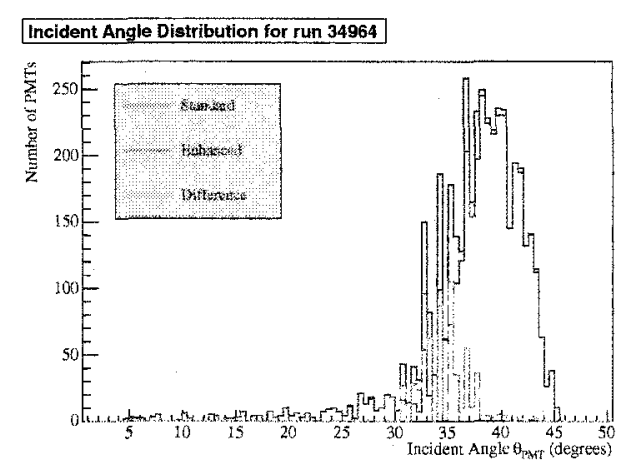

(a) $f_{\text {bad }}=0, f_{\text {cbad }}=0$.

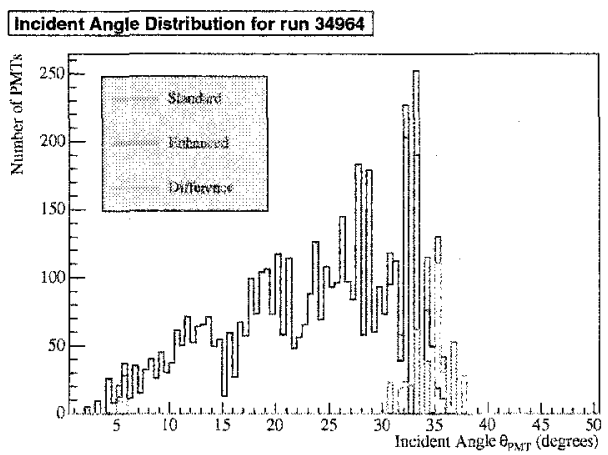

(b) $f_{\text {bad }}=1, f_{\text {pavr }}=1$.

Figure 6.2: Effect of applying the new PAVR cut on the statistics of run 34964 as a function of the incident angle $\theta_{P M T}$. Run 34964 has $R_{S}=555 \mathrm{~cm}$ in the October 2003 scan at $\lambda=500 \mathrm{~nm}$. The difference curve in each case corresponds to the change in statistics. Figure a) shows the total number of PMTs used in that run. In this case, the number of used PMTs is comparable to the number of flagged PMTs for both methods. View these plots in color. 
In Figure 6.1, the $R_{S}$ value gives access to $\theta_{P M T} \leqslant 40^{\circ}$. Figure 6.1(a) gives the number of PMTs that passed all the cuts for that run. Figure 6.1(b) shows the number of discarded tubes because of the PAVRs for both methods. The PMTs discarded using the new cut are contained in the range $\left[12^{\circ}, 28^{\circ}\right]$ which is characteristic of a band region of bad tubes on the PSUP, as it was shown in Figure 5.2. The use of the enhanced method shows a non-negligible number of recovered PMTs in the whole range of $\theta_{P M T}$. The increase for small $\theta_{P M T}$ gives access to prompt light detected by the PMTs on the opposite side of the detector with respect to the source. There is less enhancement at large $\theta_{P M T}$.

In Figure 6.2, the $R_{S}$ value gives access to $\theta_{P M T} \leqslant 45^{\circ}$. A similar discussion applies for run 34964 except that its large $R_{S}$ value reduces considerably the number of recovered PMTs using the new cut. Since the number of possible reflections per PMT per source position also increases with $R_{S}$, the number of PMTs flagged by the new PAVR cut increases with $R_{S}$, for a given time window $t_{w}$. This can be seen with the comparison of Figures 6.1(b) and 6.2(b). One can guess that above some large value of $R_{S}$, the new PAVR cut selects less PMTs than the $85^{\circ}$ cut. Hence, even if the latter cuts too much tubes within a volume of $500 \mathrm{~cm}$, it is not restrictive enough to take into account the prompt peak contamination by the AV reflections in the whole AV volume.

\subsubsection{Statistics of an Entire Scan}

For an entire scan at a specific wavelength, the effects of adding high radius runs and using the new PAVR cut can be decoupled. The effect of adding high radius runs can be isolated by applying the same processing method on different data subsets; 
the effect of using the new PAVR cut can be isolated by changing the processing method on a given data subset. Figure 6.3 shows the number of PMTs per incident angle bin after summing all the runs at $\lambda=420 \mathrm{~nm}$ for all the flag combinations of Table 6.1. The legend is different compared to Figures 6.1 and 6.2 as it identifies the processing methods and the data subsets. As previously, the enhanced method uses the new PAVR selection criterion and the standard method the $85^{\circ}$ one. The label ' + hr' means that high radius runs with $R_{S}>500 \mathrm{~cm}$ were included, otherwise they were not.

Figure 6.3(a) corresponds to the statistics obtained from PMTs with none of the flags set. The number of entries is the total number of PMTs available for the occupancy ratio fit at that wavelength. The data available dominates the range of incident angle $\left[0^{\circ}, 25^{\circ}\right]$ because most runs are taken close to the center, providing more PMTs with small $\theta_{P M T}$. The incident angle information above $40^{\circ}$ comes from using the high radius runs with $R_{S}>500 \mathrm{~cm}$.

The other distributions of Figure 6.3 all have at least one flag enabled. Figures 6.3(b), 6.3(c) and 6.3(d) show various flag combinations related to the central run used to normalize the occupancy. These figures show a similar improvement except for Figure 6.3(b). The apparent increase of the number of PMTs flagged in this figure is not real. It is a consequence of the correlation between the flag combinations. The central run has a constant number of flagged PMTs and none of them are flagged by the PAVR cut (recall it does not apply to run with small $R_{S}$ values). Thus, any decrease in one figure with $f_{\text {cbad }}=1$ shows up as an increase in another one. This is not really important for this discussion.

Figure 6.3(e) shows the PAVR cut alone. The application of the new cut adds 


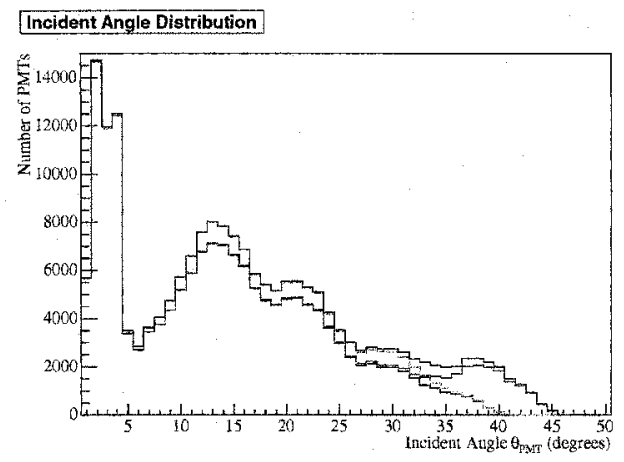

(a) $f_{\text {bad }}=0, f_{\text {cbad }}=0$.

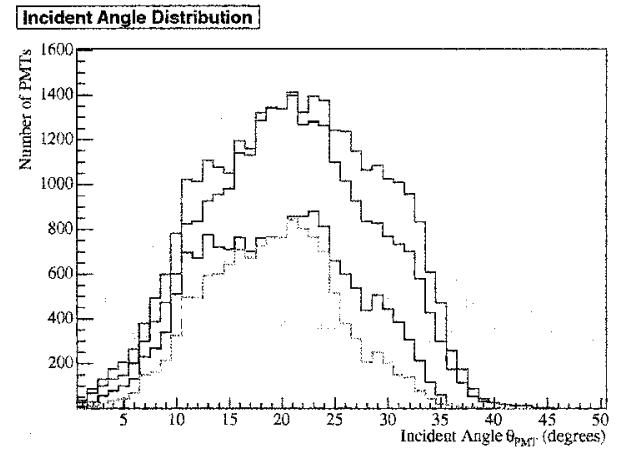

(c) $f_{\text {bad }}=1, f_{\text {cbad }}=0$.

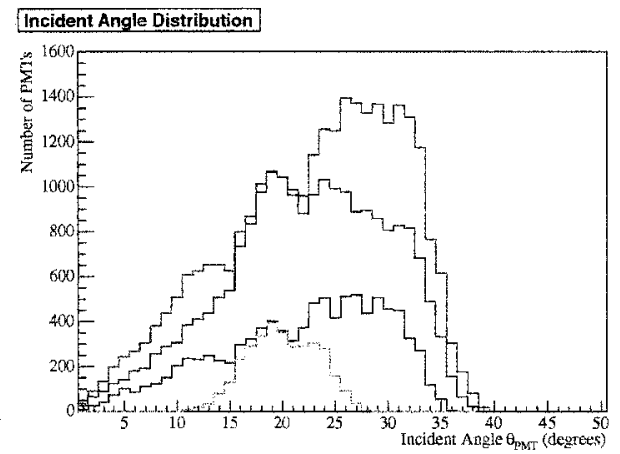

(e) $f_{\text {bad }}=1, f_{\text {pavr }}=1$.

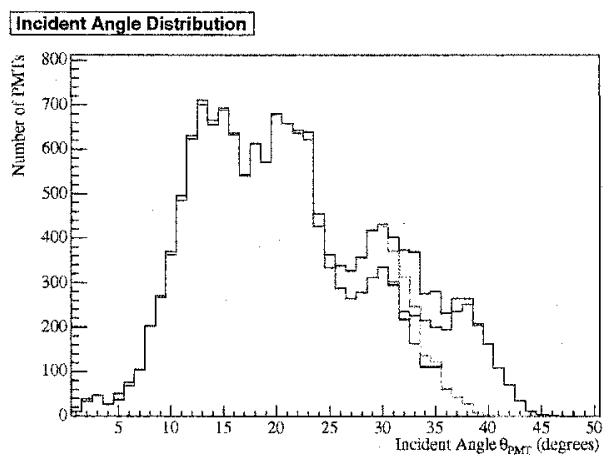

(b) $f_{\text {bad }}=0, f_{\text {cbad }}=1$.

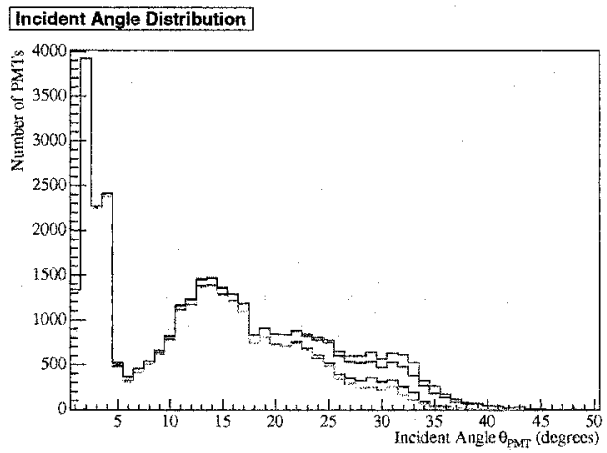

(d) $f_{\text {bad }}=1, f_{\text {cbad }}=1$.

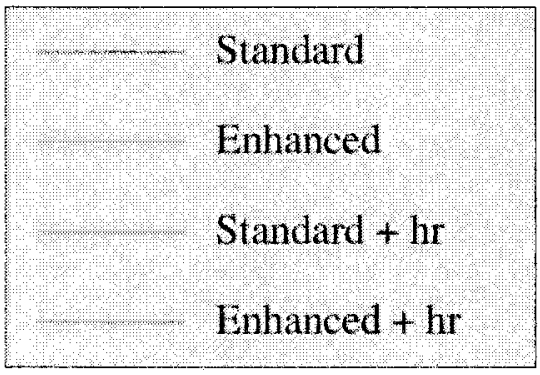

(f) Processing method legend.

Figure 6.3: Effect of applying the new PAVR cut on the statistics of the runs at $\lambda=420 \mathrm{~nm}$ from the October 2003 scan as a function of the incident angle $\theta_{P M T}$. For the occupancy ratio analysis, the PMTs with $f_{\text {bad }}=f_{\text {cbad }}=0$ are used. The processing methods are identified in the legend. The mention ' + hr' means that the runs with $R_{S}>500 \mathrm{~cm}$ have been added to the optical data set. View these plots in color. 
statistics at low and high $\theta_{P M T}$ values since the total number of PMTs in this distribution decreases. For similar data subsets, the enhanced method always flags less PMTs than the standard method in all the range of incident angle. The effect of adding high radius runs to the optical data analysis is to add more PMTs with large incident angle. These effects are similar for the other wavelengths.

The difference distribution is plotted on a logarithmic scale for $f_{\text {bad }}=f_{\text {cbad }}=0$ in Figure 6.4. It shows the change (increase) in the statistics available for the fit for this wavelength. That difference is considerable for the whole $\theta_{P M T}$ range. Both adding high radius runs and applying the new PAVR cut on the data contribute to a significant increase in the statistics. The increase in the range $\left[40^{\circ}, 45^{\circ}\right]$ comes purely from adding more high radius runs. This data provides new information about the PMTR in that range of incident angle.

\section{$6.2 \quad$ Fit Results}

The following fit results, or optical parameters also called constants, are obtained from the occupancy ratio fit of the October 2003 optical scan data. The fitting algorithm only takes the prompt light into account in the determination of a limited number of parameters from the fit to the optical data. Table 6.2 contains a summary of the fits performed. The fits are done on two different data subsets coming from the same scan but processed with two different methods, where only the selected data provided to the fitting program changes. This section focuses on the extraction of the $\mathrm{D}_{2} \mathrm{O}$ and $\mathrm{H}_{2} \mathrm{O}$ attenuations, the PMTR, and the quality of the fit. The full details of the fit results for the October 2003 scan are presented in Appendix C. 


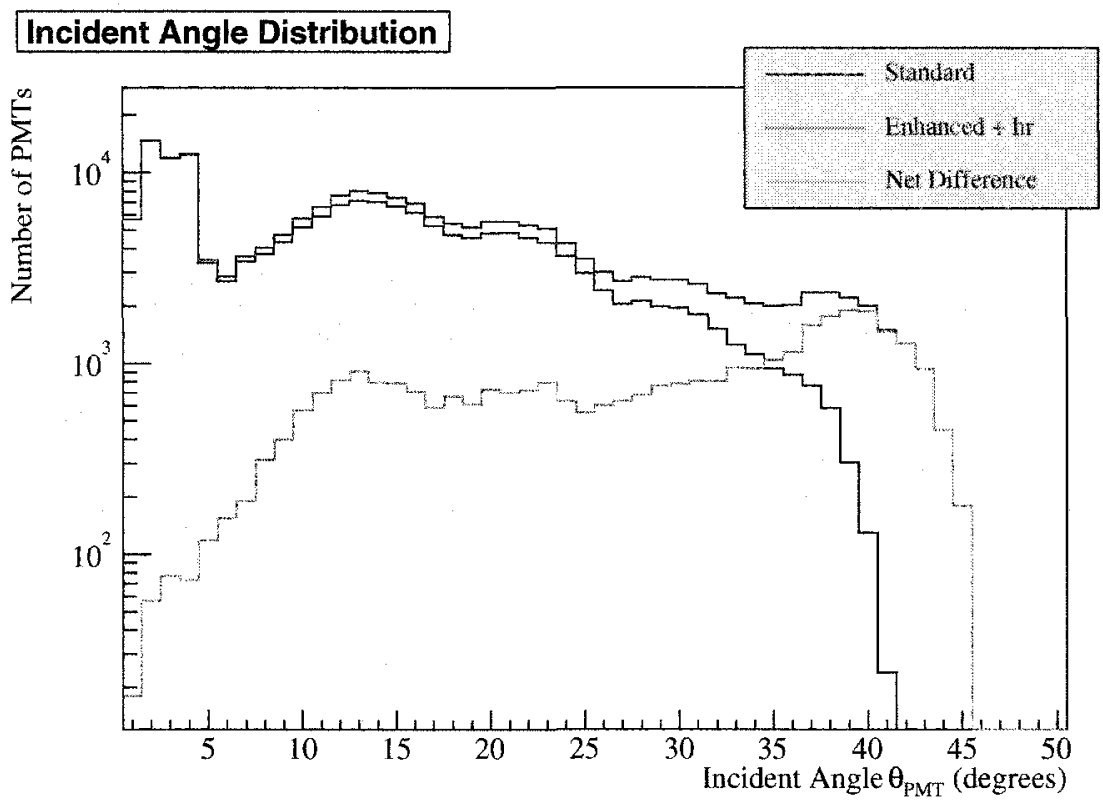

Figure 6.4: Net difference in statistics between the enhanced and the standard processing methods for runs at $\lambda=420 \mathrm{~nm}$ from the October 2003 scan as a function of the incident angle $\theta_{P M T}$. The distributions corresponds to $f_{\text {bad }}=f_{\text {cbad }}=0$. The difference is shown on a logarithmic scale to emphasize the difference at low incident angles. The mention ' + hr' means that the runs with $R_{S}>500 \mathrm{~cm}$ have been added to the optical data set. View this plot in color.

The source positions need to be determined before the data is organized and processed. The source positions were taken from the $\lambda=500 \mathrm{~nm}$ runs, called fruns fits. The position determination is better at $\lambda=500 \mathrm{~nm}$ because the phototube calibration (PCA) is done at that wavelength [20]. The manipulator positions are fixed from one wavelength to another, which enables the positions to be selected from a single wavelength. For high radius source positions, the fruns position fits are usually more precise $[7,21]$. 


\begin{tabular}{|c|c|c|c|}
\hline \hline Scan Acronym & Processing Method & PAVR Cut & $R_{S}^{\max }(\mathrm{cm})$ \\
\hline \hline oct03 & Standard & $85^{\circ}$ (default) & 500 \\
\hline oct03hnew & $\begin{array}{c}\text { Enhanced with more } \\
\text { high radius runs }\end{array}$ & $R_{S^{-} \text {dependent (new) }}$ & 555 \\
\hline
\end{tabular}

Table 6.2: Processing methods for the October 2003 scan. The $R_{S}^{m a x}$ value is fixed to $500 \mathrm{~cm}$ for the standard method since it does not use all the high radius runs available in the scan. High radius runs with $R_{S}>500 \mathrm{~cm}$ are added to use with the enhanced method. The highest radius is approximately $555 \mathrm{~cm}$.

\subsubsection{Uncertainties}

The parameter with its total uncertainty is the optical constant measurement. The total uncertainty of a parameter is the combination of the statistical and the systematic errors, added in quadrature. The statistical uncertainty depends on the amount of data available for the fit. The systematics are found by looking at a given parameter variation from its nominal value when the conditions of a run are artificially altered. Table 6.3 summarizes the systematic uncertainty smearing procedures. Each alteration affects the different parameters differently so a relative weighting of the systematics has to be done. The contribution of each systematic on the total systematic uncertainty was determined in [1].

The systematic uncertainty usually dominates the total uncertainty at high radius positions since most of the systematics are position dependent. This comes from the difficulty for the processing code to fit accurately the source positions. Thus, the addition of high radius runs should produce an increase in the systematic errors. But, based on the increase of the statistics observed in the previous section, it should also reduce the statistical uncertainties. Therefore, the total uncertainty 


\begin{tabular}{|c|c|}
\hline Systematic & Calculation \\
\hline \hline Radial scale shift & $R_{S}^{\prime}=1.01 R_{S}$ \\
$z$ position shift & $z^{\prime}=z+5 \mathrm{~cm}$ \\
$x$ position shift & $x^{\prime}=x+5 \mathrm{~cm}$ \\
Radial position shift & $R_{S}^{\prime}=R_{S}+5 \mathrm{~cm}$ \\
Radial position smear & $R_{S}^{\prime}=R_{S}+$ Gaus $(0,5 \mathrm{~cm})$ \\
Laserball-PMT distance shift & $d^{\prime}=d-3 \mathrm{~cm}$ \\
Flat laserball distribution & $L_{i j}^{\prime}=1$ \\
Squared laserball distribution & $L_{i j}^{\prime}=L_{i j}^{2}$ \\
PMT variability turned off & $\sigma_{i j}=0$ \\
Restricted $\chi^{2}$ & $\chi^{2}<16$ \\
Restricted $\chi^{2}$ & $\chi^{2}<9$ \\
\hline \hline
\end{tabular}

Table 6.3: Systematic uncertainties and their smearing procedures applied against the data. The restricted $\chi^{2}$ systematics reject the PMTs with large $\chi^{2}$.

of a parameter is not expected to change much from fitting the data processed with one method or the other.

\subsubsection{Media Attenuations}

The measurement of the $\mathrm{D}_{2} \mathrm{O}$ and $\mathrm{H}_{2} \mathrm{O}$ attenuation coefficients (or inverse attenuation lengths) obtained from the two processing methods are shown in Figures 6.5 and 6.6, respectively. Note that the values extracted from the fit correspond to the total extinction in the given medium. For the $\mathrm{H}_{2} \mathrm{O}$, it corresponds to the total extinction of the $\mathrm{H}_{2} \mathrm{O}$ plus the acrylic. The acrylic attenuation coefficient measurements were made ex situ. This parameter, $\alpha_{\mathrm{a}}$, was not allowed to vary in the fit.

In Figure 6.5, the combined effect of adding high radius runs and using the new PAVR cut in the data processing is a slight decrease of the $\mathrm{D}_{2} \mathrm{O}$ attenuation coefficients. The newly processed data gives a clearer $\mathrm{D}_{2} \mathrm{O}$ volume, but the results 


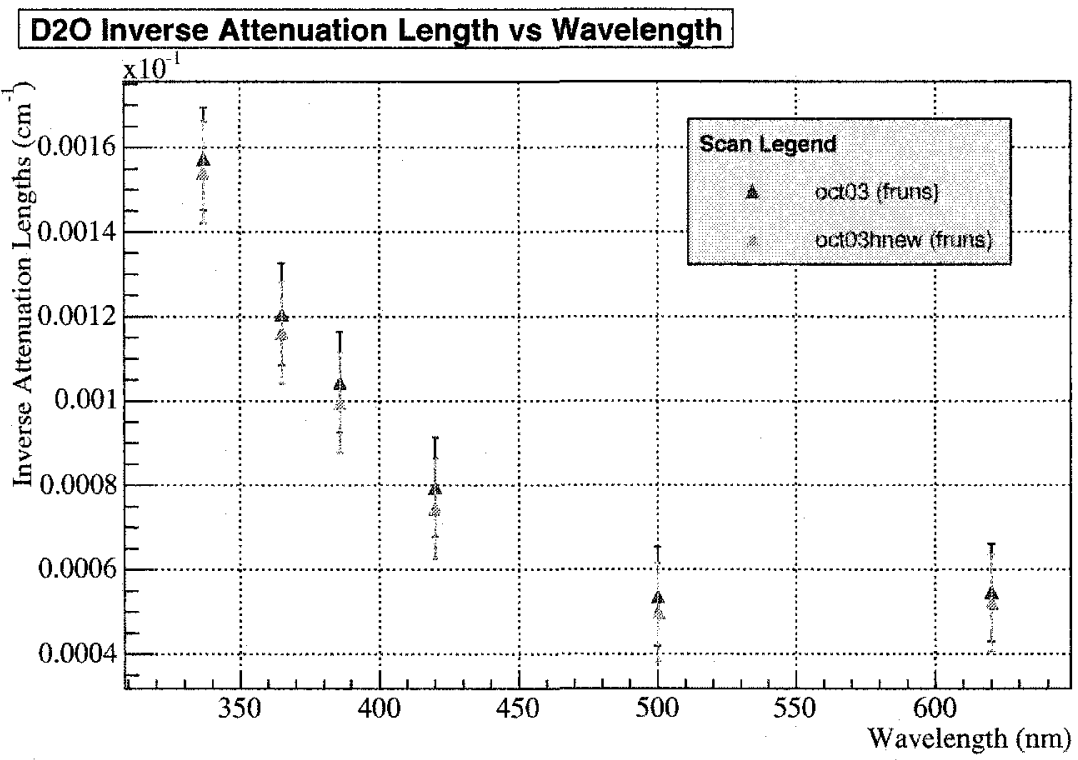

Figure 6.5: $D_{2} \mathrm{O}$ attenuation as a function of wavelength for the October 2003 scan. See Table 6.2 for the meaning of the legend acronyms.

\section{H2O Inverse Attenuation Length vs Wavelength}

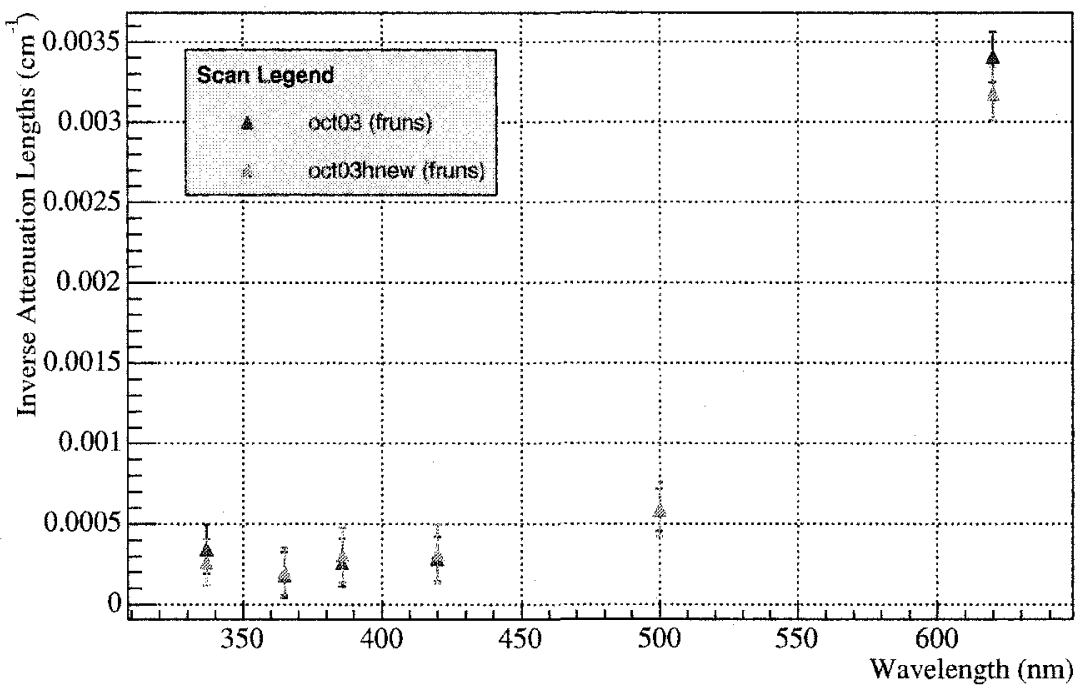

Figure 6.6: $\mathrm{H}_{2} \mathrm{O}$ attenuation as a function of wavelength for the October 2003 scan. See Table 6.2 for the meaning of the legend acronyms. 
are consistent with the previous measurements. The maximum decrease is about $3 \%$ at $\lambda=500 \mathrm{~nm}$, and the uncertainties are of the same order. The small variations of the attenuation can only be explained by an extended sampling of the $\mathrm{D}_{2} \mathrm{O}$ volume since the trend seems to be the same for all wavelengths.

In Figure 6.6, the combined effect of adding high radius runs and using the new PAVR cut in the data processing is not really significant. The difference is up to $7 \%$ at $\lambda=620 \mathrm{~nm}$, but the $\mathrm{H}_{2} \mathrm{O}$ combined to the acrylic attenuations remain consistent with what was previously measured. As for the $\mathrm{D}_{2} \mathrm{O}$ attenuations, the uncertainties are of the same order.

\subsubsection{PMT Angular Response}

The measurements of the PMTR at $\lambda=500 \mathrm{~nm}$ are shown in Figure 6.7. The PMTR extracted from the fit is a ratio of the off-center run PMT response normalized to the central run response for the same PMT. The PMTs detect the incoming light with an incident angle of $0^{\circ}$ for central runs, and with different incident angles for off-center runs, depending on the source position. Then, the relative PMTR is set to 1 at $0^{\circ}$ by definition. The empty bins are also given a default value of 1 . The increase of the response with incident angle is mainly due to the reflectors.

The combined effect of adding high radius runs and using the new processing method is a slight increase of the PMTR in the range $\left[20^{\circ}, 40^{\circ}\right]$. The PMTR found in the range $\left[40^{\circ}, 45^{\circ}\right]$ is purely due to the high radius runs. The measurements from the two methods are still consistent with each other. The increase goes up to $2 \%$ at $\theta_{P M T}=38^{\circ}$. The variations observed can be explained by the addition of new PMT data to the fit. There is more data per bin but more importantly, the data 


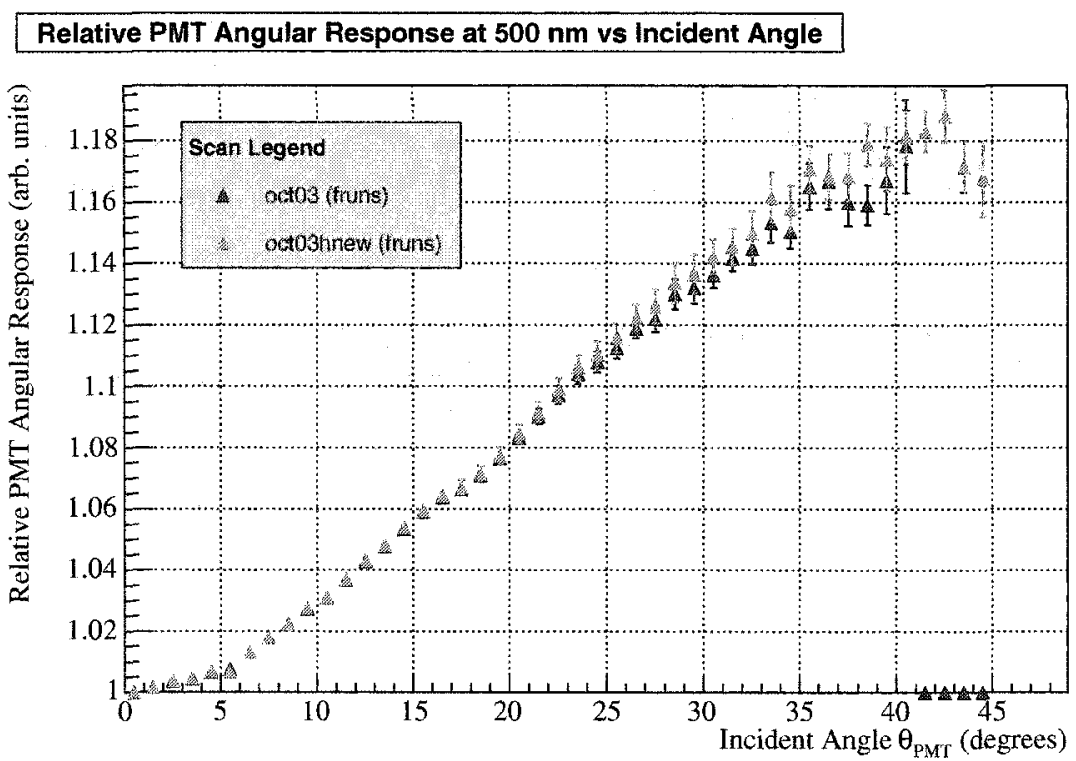

Figure 6.7: PMT angular response at $\lambda=500 \mathrm{~nm}$ as a function of incident angle for the October 2003 scan. See Table 6.2 for the meaning of the legend acronyms.

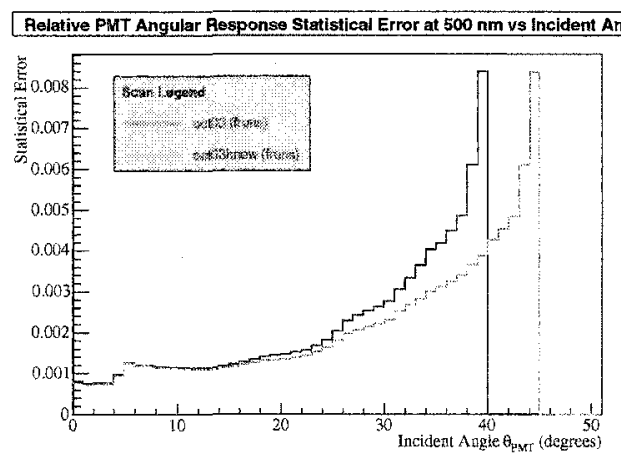

(a) Statistical errors.

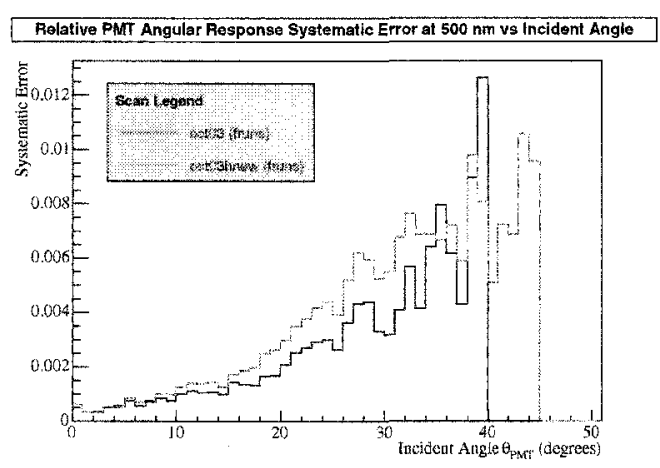

(b) Systematic errors.

Figure 6.8: Statistical and systematic errors of the PMT angular response at $\lambda=$ $500 \mathrm{~nm}$ for the October 2003 scan. See Table 6.2 for the meaning of the legend acronyms. 
added comes from different PMTs. The PMTR measurements shown in Figure 6.7 are averaged over all the PMTs for each bin. The 9438 different PMTs of the array are not expected to respond the exact same way to the light with different incident angles. Therefore, the variations are a consequence of sampling new PMTs. This is called the PMT variability. It is taken into account with the $\sigma_{i j}$ term inserted in the $\chi^{2}$ of Equation (1.8). Hence, the standard method uses a limited number of high radius runs and causes the PMTR measurements to be biased at large incident angles since the latter are obtained with a limited subset of PMTs.

The total PMTR uncertainty obtained from the enhanced method is about the same as it was for the standard method. The statistical and systematic uncertainties are shown separately in Figure 6.8. Figure 6.8(a) shows a decrease of the statistical error for the new method since there is more data per bin of incident angle. The variation of the statistical error per incident angle bin is consistent with the expectations. Figure 6.8(b) shows an increase of the systematic error for the new method since more high radius runs are processed. The variation of the systematic error for most incident angle bins is also consistent with the expectations. This is due to the fact that most systematics depend on the accuracy to determine the source position, which is not always precise at high radius. There are bins where both errors decrease: at $40^{\circ}$, the statistical error is reduced by $50 \%$ and the systematic error by $33 \%$. Overall, the total uncertainty per incident angle bin for the enhanced method is slightly smaller than what was obtained with the standard method. The PMTR for all six wavelengths can be found in Appendix C. 


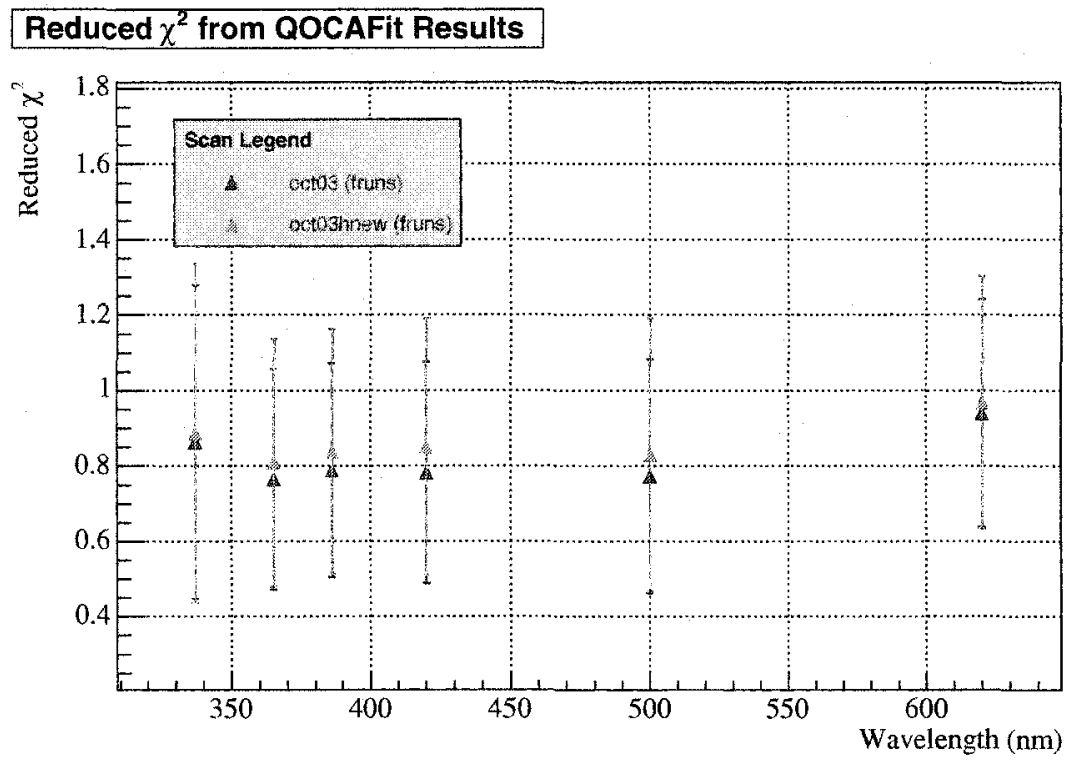

Figure 6.9: Reduced $\chi^{2}$ obtained from the optical data fits. The error bars on the values are indicators of the deviation caused by the systematic uncertainties on the input parameters. See Table 6.2 for the meaning of the legend acronyms.

\subsubsection{Quality of the Fits}

Figure 6.9 shows the reduced $\chi^{2}$, found from Equation (1.8), as a function of wavelength for the different processing methods. The quality of the fit is comparable for both methods since the fits reach convergence with a good accuracy. The error bars indicate the dispersion of the $\chi^{2}$ when the input parameters are altered according to the systematics of Table 6.3. The error bars are wider for the enhanced method. It is a consequence of the limited precision in the source position determination and the PMT variability. 


\subsection{Uncertainties due to the Optics}

When a neutrino event is assigned an energy, there are multiple systematic errors considered in the calculation of the total energy uncertainty. One of them is the uncertainty due to the optics. This contribution is dependent on the method used to measure the detector optical constants. The energy response uncertainty is found from the fit results of both processing methods to verify if the energy is more precise when more volume is calibrated. This section summarizes how to obtain an estimate of the optics contribution to the energy uncertainty when the errors of the optical parameters are propagated to the energy estimator.

\subsubsection{The Detector Response Function}

The SNO collaboration uses an energy response function (RSP) to transform the amount of detected Cerenkov light into energy. The energy estimator in SNO is based on the number NHIT of PMTs hit in an event. If the intensity of the Čerenkov light is low the PMT hits are primarily single photoelectron detection. The crucial constant measurements to be made for the energy estimator are the media attenuations and PMTR. The $\mathrm{D}_{2} \mathrm{O}$ and $\mathrm{H}_{2} \mathrm{O}$ attenuations can be measured with any set of runs [22], but it was shown that the measurements are better when greater distances are sampled inside the $\mathrm{D}_{2} \mathrm{O}$ volume. The PMTR parameters are the most important radius dependent parameters of the optical model. The PMTR at high incident angle cannot be measured unless there are high radius runs provided to the fit.

The detector fiducial volume radius $R_{\mathrm{fid}}$ sets the limit for SNO to measure the 
event energies without having to deal with low energy background coming from the $\mathrm{H}_{2} \mathrm{O}$ volume. The maximum source radius $R_{S}^{\max }$ is obtained from the highest radius of all the runs in a scan. $R_{S}^{\max }$ (or $R_{\mathrm{fid}}^{\mathrm{opt}}$ ) defines the limit where the detector optical constants can be accurately measured; $R_{\text {fid }}$ is the SNO analysis limit for neutrino event selection. Therefore, the goal of the optical calibration is to provide precise optical constants for $R_{S}^{\max } \simeq R_{\mathrm{fid}}^{\mathrm{opt}} \geqslant R_{\mathrm{fid}}$.

\subsubsection{Uncertainty on the Energy Scale}

The approximated volume-weighted uncertainty in the energy scale was determined in [1] using the difference in volume between the fiducial and the optically calibrated volume:

$$
\begin{aligned}
\Delta \operatorname{RSP}\left(R_{\mathrm{fid}}\right) \simeq & \Delta \operatorname{RSP}\left(r<R_{S}^{\max }\right) \\
& +\Delta \operatorname{RSP}\left(r>R_{S}^{\max }\right) \times\left(\frac{R_{\mathrm{fid}}^{3}-\left(R_{S}^{\max }\right)^{3}}{R_{\mathrm{fid}}^{3}}\right),
\end{aligned}
$$

where the $\Delta \operatorname{RSP}\left(r>R_{S}^{\max }\right) \sim 5 \%$ in the range $\left[40^{\circ}, 50^{\circ}\right]$. This factor is estimated to be the difference between the ex situ PMTR measurements given in [23] and the SNOMAN extrapolation based on in situ measurements (fits). It is multiplied by the volume fraction that has not been covered by the source positions in a scan. The effect of the systematic uncertainties of Table 6.3 is approximately $0.1 \%$ in the volume where the optical constants have been measured [1]. Applying this information to Equation (6.1), the energy scale total uncertainty due to the optical parameters is calculated for two fiducial volume radii, $R_{\mathrm{fid}}=550 \mathrm{~cm}$ and $R_{\mathrm{fid}}=600$ $\mathrm{cm}$, for both sets of optical parameters obtained. Table 6.4 summarizes the results. 


\begin{tabular}{|c|c|c|c|c|c|}
\hline $\begin{array}{c}\text { Processing } \\
\text { Method }\end{array}$ & $\begin{array}{l}R_{\mathrm{fid}} \\
(\mathrm{cm})\end{array}$ & $\begin{array}{c}\Delta \mathrm{RSP} \\
\left(r<R_{S}^{\max }\right)\end{array}$ & $\begin{array}{c}\Delta \mathrm{RSP} \\
\left(r>R_{S}^{\max }\right)\end{array}$ & $\left(\frac{R_{\mathrm{fid}}^{3}-\left(R_{S}^{\max }\right)^{3}}{R_{\text {fid }}^{3}}\right)$ & $\begin{array}{l}\Delta \mathrm{RSP} \\
\left(R_{\mathrm{fid}}\right)\end{array}$ \\
\hline $\begin{array}{c}\text { Standard } \\
\left(R_{S}^{\max }=500 \mathrm{~cm}\right)\end{array}$ & $\begin{array}{l}550 \\
600\end{array}$ & $\begin{array}{l}\sim 0.13 \% \\
\sim 0.16 \%\end{array}$ & $\begin{array}{l}5 \% \\
5 \%\end{array}$ & $\begin{array}{l}24.9 \% \\
42.1 \%\end{array}$ & $\begin{array}{l}\sim 1.37 \% \\
\sim 2.26 \%\end{array}$ \\
\hline $\begin{array}{c}\text { Enhanced }+\mathrm{hr} \\
\left(R_{S}^{\max }=550 \mathrm{~cm}\right)\end{array}$ & $\begin{array}{l}550 \\
600\end{array}$ & $\begin{array}{l}\sim 0.13 \% \\
\sim 0.16 \%\end{array}$ & $\begin{array}{l}5 \% \\
5 \%\end{array}$ & $\begin{array}{l}0.0 \% \\
22.3 \%\end{array}$ & $\begin{array}{l}\sim 0.13 \% \\
\sim 1.27 \%\end{array}$ \\
\hline Improvement & $\begin{array}{l}550 \\
600\end{array}$ & $\begin{array}{l}0 \% \\
0 \%\end{array}$ & $\begin{array}{l}0 \% \\
0 \%\end{array}$ & $\begin{array}{l}24.9 \% \\
19.8 \%\end{array}$ & $\begin{array}{l}\sim 1.25 \% \\
\sim 1.00 \%\end{array}$ \\
\hline
\end{tabular}

Table 6.4: Energy scale uncertainties due to the optics for different processing methods and fiducial volumes. The total uncertainties for the standard and the enhanced methods are of the same order. Then, the terms $\Delta \operatorname{RSP}\left(r<R_{S}^{\max }\right)$ are also of the same order. The improvement of the enhanced method is mainly caused by the increase of the value of $R_{S}^{\max }$, or the decrease of the volume weighted factor.

The improvement on the energy scale uncertainty is of the order of $1.25 \%$ and $1.00 \%$ for $R_{\mathrm{fid}}=550 \mathrm{~cm}$ and $R_{\mathrm{fid}}=600 \mathrm{~cm}$, respectively. As explained earlier, the optical fit systematic uncertainties are kept at the same level by using the new PAVR selection criterion even if more high radius runs are added. Hence, the net improvement is caused by the increase of $R_{S}^{\max }$ combined with the use of the enhanced processing method. 


\section{Chapter 7}

\section{Discussion and Conclusion}

This final chapter contains a discussion on the impact of the new proposed way of selecting the data before finding the optical constants for the SNO solar neutrino data analysis. The optical calibration methods and analyses are discussed for the next phase of the experiment, the neutral current detector (NCD) phase. The thesis concludes on what was accomplished and what work remains to be done to obtain even more precise measurements of the optical parameters.

\subsection{More on the Enhanced Method}

The AV reflection analytic model assumes that the existing code and the geometry used by the SNO collaboration are accurate tools to extract the optical constants. The enhanced processing method discards the PMTs that detect AV reflected light based on that model. The input parameters needed for the new selection criterion and the uncertainties related to this procedure are discussed here. 


\subsubsection{Time Windows}

The time window parameter $t_{w}=8 \mathrm{~ns}$ used with the enhanced method is equivalent to a $97.7 \%$ AV reflection rejection, based on the prompt time window of \pm 4 ns centered on the mean time of the prompt peak in the optical data analysis. The choice of 8 ns removes the PMTs contaminated by PAVRs with $\Delta t<t_{w}$, but allows $2.3 \%$ of the total amount of reflected light to be detected by a PMT. The reflections with small time differences are mostly LI reflections. The probability for these reflections to happen is already negligible given a small Fresnel reflection coefficient, which gives a possible contamination of $2.3 \%$ of a negligible light intensity. Wider time windows could be used to remove up to $99 \%$ of the reflections but too wide time windows bring the analysis back to what is was with the old PAVR cut. This choice must keep the reflections away from the prompt peak, but must ensure a sufficient number of PMTs are being sampled, so that an unbiased calibration is made.

\subsubsection{Uncertainties}

The new QOCAReflect code is inserted in the processing chain to calculate the limits on $\cos \theta_{p s}$ based on the parameters $R_{S}$ and $t_{w}$, so that QOptics can flag the PMTs detecting PAVRs in a radius dependent manner for each run. Most of the systematic uncertainties are calculated by allowing an artificial displacement of the source from its original position. The limits on $\cos \theta_{p s}$ are also calculated for the systematics, incorporating the effect of the AV reflections. The position uncertainty is then translated into an uncertainty on the number of PMTs flagged for PAVRs: more or less PMTs survive the PAVR cut. Assuming the time window parameter is set to a conservative value, no further significant checks need to be made. 


\subsection{Comparison of the Optical Constants}

The optical model fit gives back a set of optical constants characterizing the SNO detector. These constants are needed to estimate the energy response of the detector as a function of the number of PMT hits seen in a neutrino event. The constants of the October 2003 scan were extracted after processing the data in two different ways. The modifications made to the standard method led to new optical constants. The new constants do not change significantly from the old ones as the physical properties of the detector remain the same when the data comes from the same scan. Any method used, if correct, should give consistent results. Nevertheless, variations up to $3 \%$ for the $\mathrm{D}_{2} \mathrm{O}$ attenuation, up to $2 \%$ for the $\mathrm{H}_{2} \mathrm{O}$ attenuation, and up to $2 \%$ for the PMTR at $\lambda=500 \mathrm{~nm}$ were observed. The causes of these variations are discussed here, keeping in mind that the variations are small and that the fit results obtained are consistent with the previous measurements of the optical parameters.

\subsubsection{Media Attenuations}

There is one attenuation coefficient extracted per wavelength, which is a fixed number of parameters given the condition of a scan. Since none of the processing methods change the conditions of the scan, the attenuations obtained are consistent, otherwise the same, considering the error bars. For the $\mathrm{D}_{2} \mathrm{O}$ attenuations, the small variations observed are consistent over all wavelengths. It is explainable by a increase of the sampled volume. The previous measurements were limited to $R_{S}^{\max }=500 \mathrm{~cm}$. It corresponded to $58 \%$ of the central volume or $75 \%$ of the fiducial volume. The high 
radius runs provide calibration data up to $R_{S}^{\max }=555 \mathrm{~cm}$, which corresponds to $80 \%$ of the central volume, or even better, $100 \%$ of the fiducial volume.

\subsubsection{PMT Response}

The PMTR parametrization is different from the media attenuations. There is a variable number of parameters, the incident angle bins. If enough data is found in all the bins, the parametrization will be precise enough. Otherwise, if the conditions of a scan were bad, the PMTR obtained will be bad and of limited precision.

The PMTR was obtained from fitting the data subsets which were processed with two different methods. The results obtained are consistent over the range in incident angle common to both methods since the runs in the range $R_{S}=[0,500]$ $\mathrm{cm}$ were common to both data subsets. The addition of high radius runs for the enhanced processing methods made that range wider, since these runs have $R_{S}=$ $[500,550] \mathrm{cm}$. As expected, the PMTR extends to $\theta_{P M T}=45^{\circ}$ using those runs. But these runs also have PMTs in the whole range $\theta_{P M T}=\left[0^{\circ}, 45^{\circ}\right]$. Therefore, the small variations in the range $\left[0^{\circ}, 40^{\circ}\right]$ are due to sampling more PMTs, assuming the response of the individual tubes stay the same. The new data in the range $\left[40^{\circ}\right.$, $45^{\circ}$ comes from sampling more incident angles with more PMTs. A given PMTR parameter or incident angle bin is obtained by averaging all the responses in that bin. In a perfect optical calibration scan, enough runs would be taken such that all the PMTs could be sampled at all the incident angles. Considering the limited time SNO has to calibrate, this is impossible. The measurement made in this thesis are the best up to now since it is obtained from using the whole $\mathrm{D}_{2} \mathrm{O}$ scan data. 


\subsubsection{Uncertainties}

The optical model fit is obtained by minimizing a $\chi^{2}$. The quality of the different fits performed is the same. The statistical and the systematic errors associated with the parameters vary in opposite directions, as it was shown for the PMTR, such that the total uncertainty is of the same order. This translates into an improved detector energy response, given the smaller contribution of the optical response uncertainty. The optical constants affect the energy scale total uncertainty by $\simeq 0.1 \%$ at $R_{\text {fid }}=$ $550 \mathrm{~cm}$, an improvement of $1.25 \%$.

\subsection{Improvements Needed for the NCD Phase}

At the time of redaction of this thesis, the first laserball runs of the NCD phase were being analyzed. There are 40 NCDs deployed in the detector, parallel to the $z$-axis, in an array contained in the $x y$ plane of the detector. The presence of the NCDs affects the optics of the detector since they absorb and scatter photons, resulting in a smaller amount of prompt light detected by the PMTs [24]. This is known as the NCD shadowing effect. The light paths affected by the presence of the NCDs are flagged with a new flag, $f_{\text {ncd. }}$. Since an optics scan is performed to calibrate the media attenuations and the PMT response, the run positions of the scan must be carefully selected to minimize the number of PMT flagged by $f_{\text {ncd }}$.

\subsubsection{Fit Alternatives}

The first results of the application of the $f_{\text {ncd }}$ flag showed that the analysis is left with almost half of the tubes for a central run, an important decrease in statistics 
compared to the $\mathrm{D}_{2} \mathrm{O}$ and salt phases. The occupancy ratio method is then compromised since the chances that a PMT remains valid for both off-center and central runs is much lower than it was for the previous phases of the experiment. There are thus fewer PMTs available for the occupancy ratio fit. It is the main reason why the relative PMT efficiencies study [8] was done and why the occupancy fit of Equation (1.9) is better suited for the NCD phase. The efficiencies are measured from the salt phase data using the occupancy ratios. Hence any measurement of the efficiencies during the NCD phase still relies on the occupancy ratio method.

Using the occupancy ratio method, ignoring the presence of the NCD, affects the $\mathrm{D}_{2} \mathrm{O}$ attenuation. If no cut is made for the presence of the NCDs, the fit compensates for the lack of prompt light, absorbed by the NCDs, by increasing the $\mathrm{D}_{2} \mathrm{O}$ attenuation. This result is not very useful except to verify that the NCDs really absorb or scatter light. It is difficult to decouple the NCD contribution to the $\mathrm{D}_{2} \mathrm{O}$ attenuation using the occupancy ratio method. Hence the NCD reflectivity must be added to the optical model to account for the different optics of the detector with NCDs.

Alternatives to the occupancy ratio method are under study. The use of normalization runs away from the detector center is desirable to avoid the flag $f_{\text {cbad }}$ to affect the off-center run data. The NCD array geometry must be taken into account such that off-center and normalization runs should have different $z$ values, but the same $x$ or $y$ so that the source basically sees the same number of PMTs at different angles. The first NCD optical scan is planned [25] to contain runs on horizontal and vertical axes. These runs were included to measure the laserball distribution [26]; they could also be used as normalization runs. 


\subsection{Conclusion}

The PMT angular response was measured in the range $\left[0^{\circ}, 45^{\circ}\right]$, corresponding to a $550 \mathrm{~cm}$ radius detector fiducial volume. The measurement was possible due to the study of the prompt light contamination by AV reflections at high radius source positions. The study was found to be successful by comparing the simple AV reflection analytic model used in the SNO optics code to SNOMAN simulations.

As explained in [1], if the detector fiducial volume is greater than the optically calibrated volume, it is necessary to extrapolate the PMTR with SNOMAN in the regions where no calibration measurements were done. The extrapolation increases the fit uncertainties in the extrapolated areas, which then propagates the uncertainties in the energy scale for neutrino event detection. The uncertainties on the energy scale due to the optics depend on the extraction method of the optical parameters. The new PMTR information gives a better understanding of the detector response at high radius, which decreases the uncertainty on the energy scale due to the optical calibration. This energy uncertainty is estimated to $\sim 0.1 \%$ in the fiducial volume of SNO, $R_{\mathrm{fid}}=550 \mathrm{~cm}$, corresponding to a $1.25 \%$ decrease from the previous measurements.

There is a need to increase even more the number of bins in the PMTR measurement. This would require an analysis beyond the AV, in the surrounding $\mathrm{H}_{2} \mathrm{O}$ volume. This region of the detector has been sampled, but the data has not been included in the fits because the analyzed data has not been completely understood [27]. The reflections are expected to be quite complicated in the $\mathrm{H}_{2} \mathrm{O}$ volume because of the inverse curvature of the AV surface and the proximity to PMT reflectors. However, such a measurement would enlarge the PMTR range and provide a better 
measurement of the $\mathrm{H}_{2} \mathrm{O}$ attenuation and would allow to decouple the $\mathrm{H}_{2} \mathrm{O}$ from the acrylic attenuation.

It is also important to measure the time dependency of the PMTR because the effect of submerging the PMTs in water for a long period of time might affect their sensitivity to light. At the present time, some PMTs have deteriorated reflectors [28]. The PMTR is affected by the aging detector and that must be carefully studied.

In conclusion, an enhanced PMT selection method was used to extract the optical constants of the SNO detector using the complete $\mathrm{D}_{2} \mathrm{O}$ optical data set. The results are consistent with previous methods and an enhancement is seen for the PMTR compared to the default method used since 1999. For this reason, the enhanced method used in this thesis must replace the default method, so that the SNO detector is characterized according to the fiducial volume used for the neutrino analysis. 


\section{Appendix A}

\section{Laserdisk Command File}

The following contains the command file used in SNOMAN to run the laserdisk source simulations referred to in Chapter 4. The executable was compiled against version 4_086. The code is included in the official version of SNOMAN 4_093.

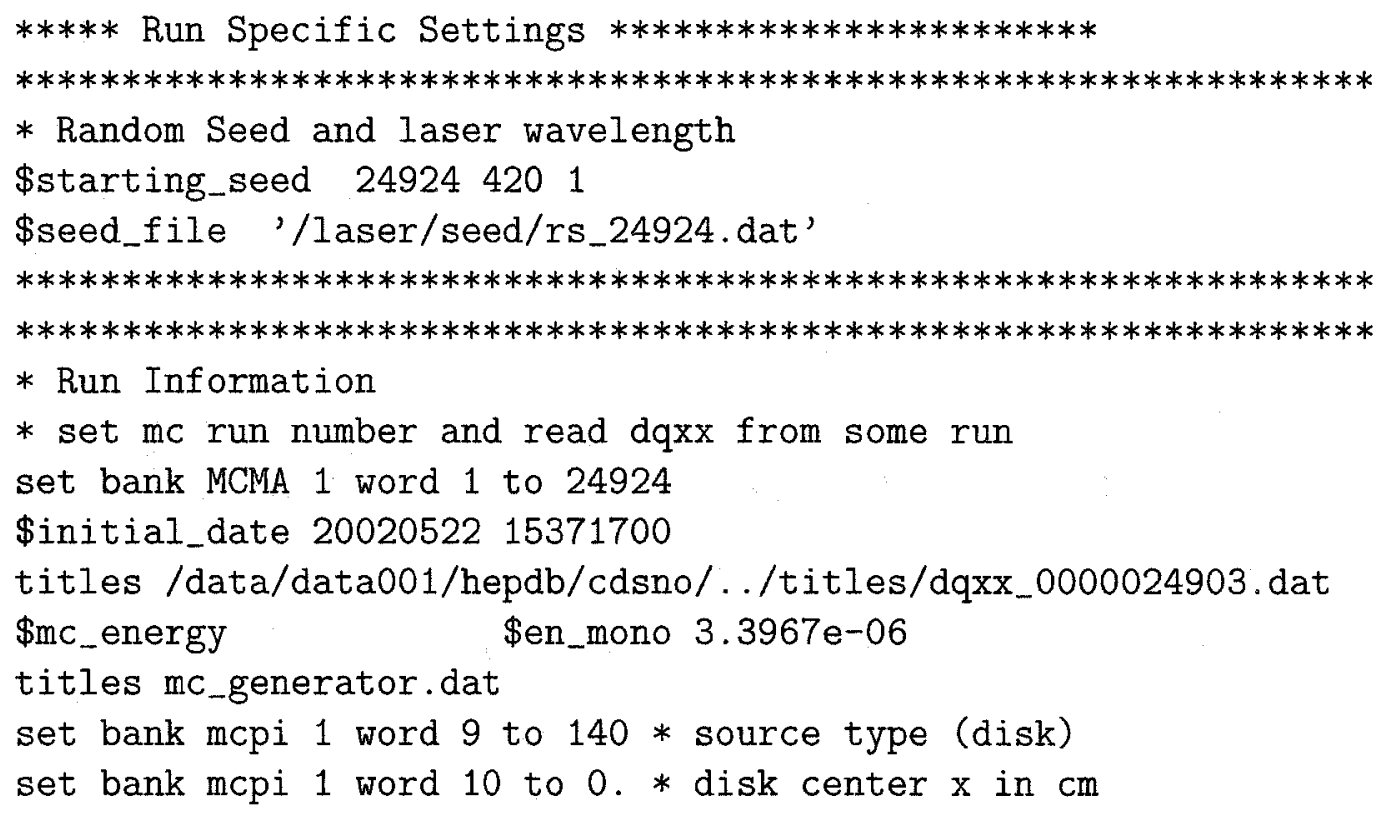


set bank mcpi 1 word 11 to 0 . * disk center $\mathrm{y}$ in $\mathrm{cm}$

set bank mcpi 1 word 12 to -500 . * disk center $z$ in $\mathrm{cm}$

set bank mcpi 1 word 13 to 10 . * disk radius in $\mathrm{cm}$

set bank mcpi 1 word 14 to $1.5708 *$ disk orientation (theta) in rad.

set bank mcpi 1 word 15 to $0.0 *$ disk orientation (phi) in rad.

set bank mcpi 1 word 20 to $20 *$ direction type (beam)

set bank mcpi 1 word 21 to $1.5708 *$ beam orientation (theta) in rad.

set bank mcpi 1 word 22 to $0.0 *$ beam orientation (phi) in rad.

$* * * * * * * * * * * * * * * * * * * * * * * * * * * * * * * * * * * * * * * * * * * * * * * * * * * * * * * * * * * * * * * * *$

* laser simulation settings

* it should match the source position above

set bank tqio 2 word 9 to 1 * use tqio position

* in time of flight subtraction

set bank tqio 2 word 10 to $0 . * x$ of source position in $\mathrm{cm}$

set bank tqio 2 word 11 to 0 . * y of source position in $\mathrm{cm}$

set bank tqio 2 word 12 to -500 . * z of source position in $\mathrm{cm}$

FILE QIO 2 /laser/rch/outputfile.rch

titles /laser/defaults/laserball_dye_default.dat

titles/laser/defaults/pmt_reflec_response_20030328.dat

titles/laser/defaults/pmt_response_qoca_20030328.dat

titles/laser/defaults/energy_calibration_20030408.dat

titles /laser/defaults/rsps_chcs_salt_replaced_24mar2003.dat

titles/laser/defaults/rsp_rayleigh_20030328.dat

titles/laser/defaults/qio_data_laser.dat

Q/laser/defaults/media_qoca_20030328.cmd

@/laser/defaults/laser_mc_defaults.cmd 


\section{Appendix B}

\section{Time Distributions}

The following contains a collection of Monte Carlo time distribution plots referred to in Chapter 4. They show explicitly the time distributions and the fit results when the Gaussian functions of Equations (4.1) and (4.2) are fitted to light peaks. Unless a specific PMT number $n$ is mentioned, the time histograms showed are the sum of all the PMT hits obtained in a MC run. There is also an additional discussion on the SNOMAN results.

\section{B.1 Laserdisk Source Radius}

This section presents how the laserdisk source radius $R_{D}$ was set for the simulations presented in Chapter 4 . An adequate $R_{D}$ value must be chosen such that the time distributions can be fitted with precision. The $R_{D}$ parameter was changed from one $\mathrm{MC}$ experiment to another, keeping all the other parameters fixed. The MC runs all have the same number of events. The time difference $\left\langle\delta t_{\text {disk }}\right\rangle$ and the widths $\sigma_{i}$ are used to compare the consistency of the different $R_{D}$ values with each other. The 


\begin{tabular}{|c|cccc|c|}
\hline \hline$R_{D}(\mathrm{~cm})$ & $\mu_{\text {ref }}$ & $\sigma_{\text {ref }}$ & $\mu_{\text {prompt }}$ & $\sigma_{\text {prompt }}$ & $\left\langle\delta t_{\text {disk }}\right\rangle$ \\
\hline \hline 5 & 0.23 & 2.757 & -29.58 & 1.143 & $29.8 \pm 2.9$ \\
10 & 0.03 & 2.514 & -29.69 & 1.003 & $29.7 \pm 2.7$ \\
20 & 12.38 & 2.773 & -15.86 & 1.683 & $28.2 \pm 3.3$ \\
30 & 9.73 & 2.717 & -19.11 & 1.157 & $28.8 \pm 2.9$ \\
40 & 9.37 & 2.793 & -19.53 & 1.205 & $28.9 \pm 3.0$ \\
50 & 12.50 & 2.909 & -16.28 & 1.338 & $28.8 \pm 3.2$ \\
100 & 28.17 & 3.712 & +0.14 & 1.714 & $28.0 \pm 4.1$ \\
\hline \hline
\end{tabular}

Table B.1: Gaussian fit parameters and time differences (in ns) between reflection and prompt light peaks for different laserdisk radius sizes. The means $\mu$ and the widths $\sigma$ are found via a fit using Equation (4.2).

peak widths $\sigma_{i}$ are used to quantify the spread of the peak mean times $\mu_{i}$.

As in Figure 4.3, the laserdisk was positioned on the negative $z$-axis at $(x, y, z)$ $=(0,0,-550) \mathrm{cm}$ shining towards the bottom of the $\operatorname{AV}\left(\theta_{\text {disk }}=\pi, \phi_{\text {disk }}=0\right)$. The disk radius $R_{D}$ was incremented by large amounts, from $5 \mathrm{~cm}$ to $100 \mathrm{~cm}$, to look at the possible effect on the prompt and reflection light peaks. The Equation (4.2) is fitted to the peaks for each value of $R_{D}$ tested. The time difference $\left\langle\delta t_{\text {disk }}\right\rangle$ is found using Equation (4.6) and the error on $\left\langle\delta t_{\text {disk }}\right\rangle$ is given by Equation (4.4). Table B.1 summarizes the fit results which are explicitly shown for each individual distribution in Figure B.1. Figure B.2 shows the different time distributions obtained for the same values of $R_{D}$ when corrected for the mean time of the prompt peak. The corrected time is $t-\mu_{\text {prompt }}$ so that the prompt peaks are all centered at $t=0 \mathrm{~ns}$. From that comparison plot, it can be seen that by increasing $R_{D}$ the reflection peaks contain less statistics and have a larger spread compared to the prompt peak.

The disk radius does not affect significantly the time difference $\left\langle\delta t_{\text {disk }}\right\rangle$ for a given source position and a given disk orientation. However, the dispersion of the 


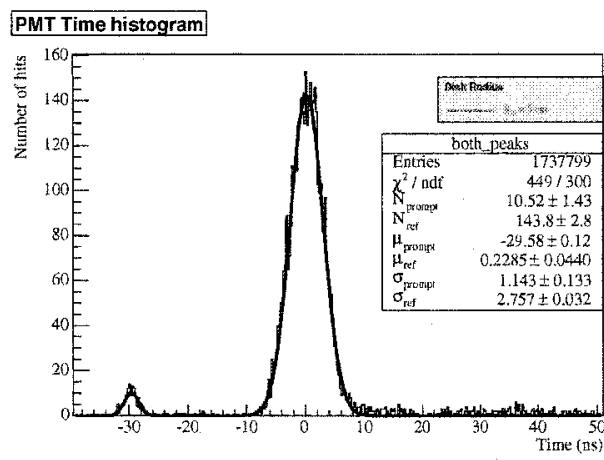

(a) $R_{D}=5 \mathrm{~cm}$.

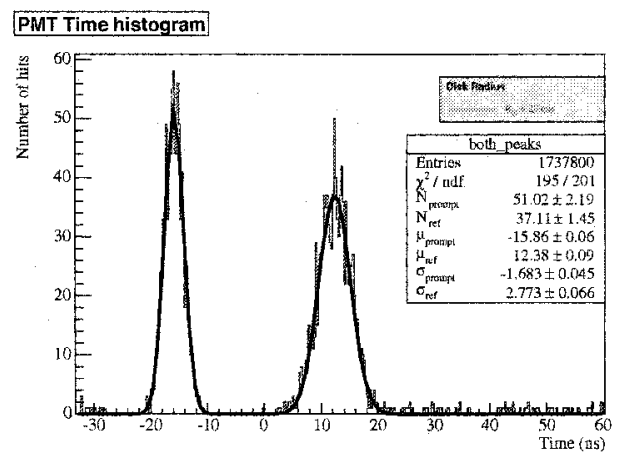

(c) $R_{D}=20 \mathrm{~cm}$

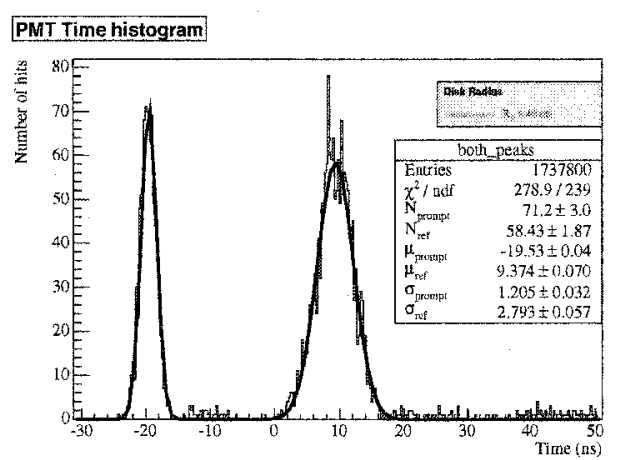

(e) $R_{D}=40 \mathrm{~cm}$

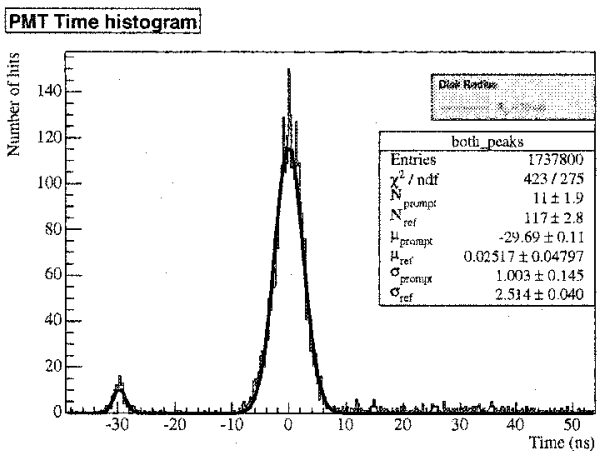

(b) $R_{D}=10 \mathrm{~cm}$.

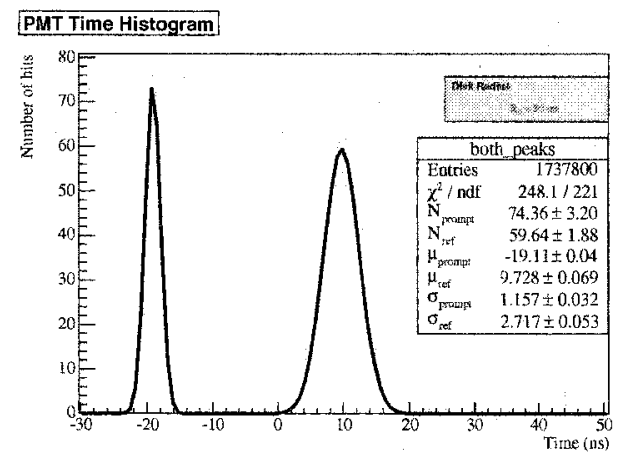

(d) $R_{D}=30 \mathrm{~cm}$.

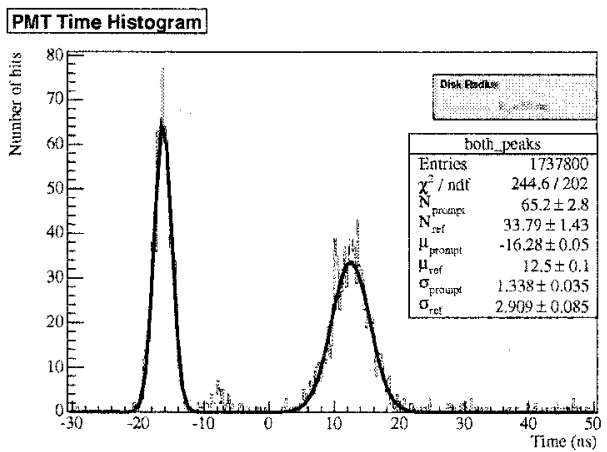

(f) $R_{D}=50 \mathrm{~cm}$.

Figure B.1: Monte Carlo laserdisk source PMT time distributions and fits to double Gaussian functions. The source position is $(x, y, z)=(0,0,-550) \mathrm{cm}$. For each disk radius, the beam direction is $\theta_{\text {disk }}=\pi, \phi_{\text {disk }}=0$. On each plot, the left peak corresponds to the prompt peak and the right peak is the reflection peak. Other small peaks for larger $R_{D}$ values are effects of the radius size. 


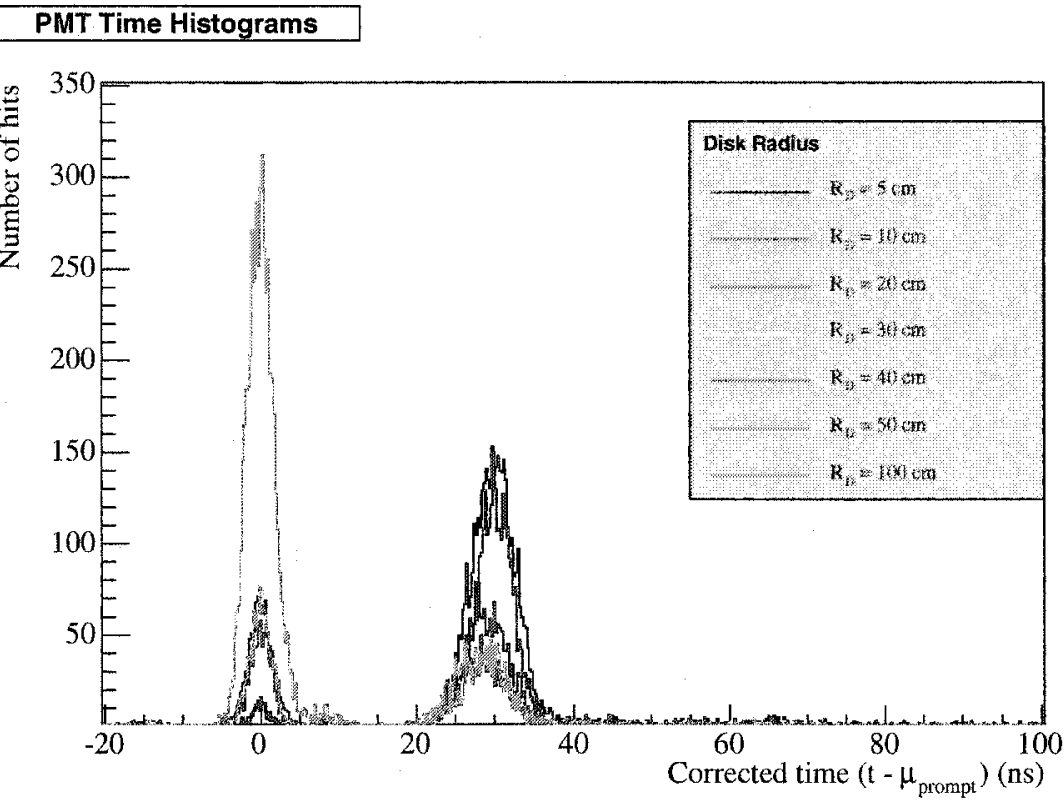

Figure B.2: Monte Carlo laserdisk source PMT time distributions for different radius sizes. The source position is $(x, y, z)=(0,0,-550) \mathrm{cm}$. For each disk radius, the beam direction is $\theta_{\text {disk }}=\pi, \phi_{\text {disk }}=0$. The raw time of each distribution is corrected for the prompt peak mean time $\mu_{\text {prompt }}$ in Table B.1 so that the peaks have the same time reference. View this plot in color.

reflection peak $\sigma_{\text {ref }}$ increases with $R_{D}$. This is consistent with the fact that the source is less focused for large values of $R_{D}$. Another consequence of increasing $R_{D}$ is the appearance of secondary peaks (see Figure B.1(f) at $t \approx-8 \mathrm{~ns}$ ). It is an effect of the radius size since the curvature of the AV affects the photons coming from the edges of the laserdisk differently. Hence, $R_{D}$ must remain as small as possible. A good value must be a compromise between a focused source and a large enough beam to get sufficient statistics. For these reasons, the laserdisk source radius used in the $\mathrm{MC}$ runs of Chapter 4 is

$$
R_{D}=10 \mathrm{~cm}
$$


This value provides the smallest $\sigma_{\text {ref }}, \sigma_{\text {prompt }}$, and $\sigma_{\left\langle\delta t_{\text {disk }}\right\rangle}$. As a comparison with the laserball used in optical calibrations, this radius is twice as large as the size of the laserball radius $\left(R_{L B} \simeq 5.5 \mathrm{~cm}\right)$.

\section{B.2 Time Distribution Fits}

Figure B.3 shows the light peaks obtained from the different laserdisk configurations of Chapter 4 . The prompt and reflected light peaks are isolated using $N^{\text {hit }}$ filters as explained in Section 4.5. The filters are efficient at separating the peaks. Figure B.4 shows the same peaks of Figure B.3 separately to get the individual peak fit results. Figure B.5 shows the photon bomb time distributions for the same configurations in both regular and logarithmic scales. The values obtained in both Figures B.4 and B.5 are used to calculate the time differences estimators $\langle\Delta t\rangle$ of Equation (4.10). Figure B.6 shows laserdisk individual PMT time distributions with fit results. Finally, Figure B.7 is the photon bomb prompt peak data, selected from the $N_{T}$ PMTs of Table 4.2, for each configuration. It shows that the use of a selected number of PMTs from the photon bomb data reduces the precision of the fit.

\section{B.3 Comments on SNOMAN}

\section{B.3.1 Laserball Source}

The laserball and the photon bomb sources are very similar in the way they generate the photons. The laserball source is more specific to optical calibrations. Work has been done and is still in progress to get an efficient laserball source that reproduce 


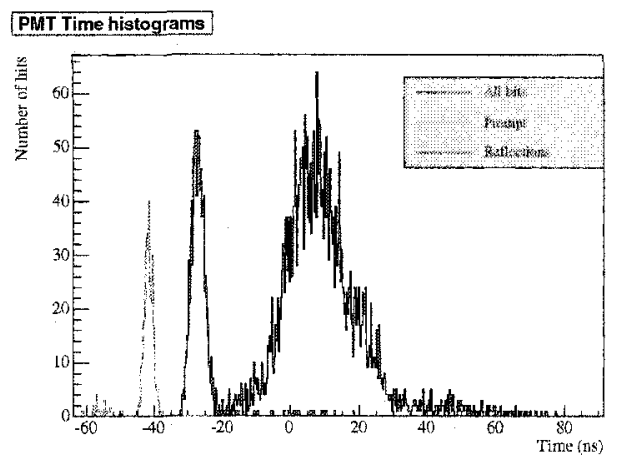

(a) Laserdisk (A) $R_{S}=475 \mathrm{~cm}$.

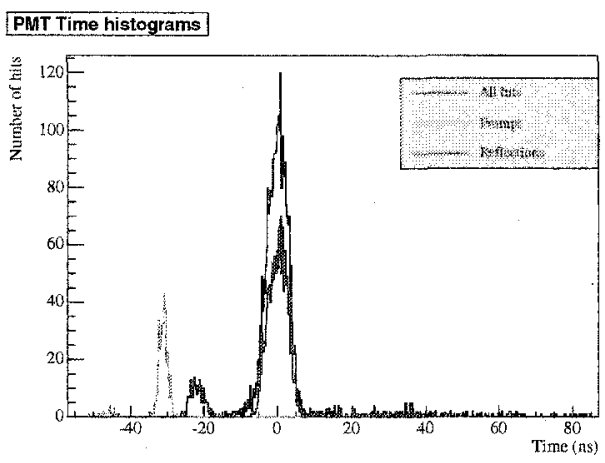

(b) Laserdisk (B) $R_{S}=550 \mathrm{~cm}$.

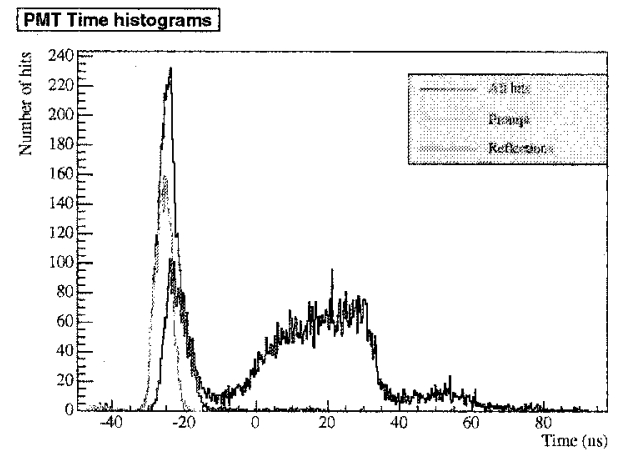

(c) Laserdisk (C) $R_{S}=575 \mathrm{~cm}$.

Figure B.3: Monte Carlo PMT time distributions for various laserdisk source configurations used for the comparisons to the analytic model. The configuration is indicated between parentheses. The prompt and reflection peaks are isolated according to the data filters described in Table 4.2. View these plots in color.

confidently the data obtained with the real source during optical calibrations. In SNOMAN, this source type should try to reproduce the real laserball asymmetry, its mask, and its spherical shape. The direction of each laserball surface photon should be selected out of a $2 \pi$ solid angle such that the orientation of the ray vary from the normal to the surface to its tangent [29]. The probability of each photon to come out of the surface should be weighted by the angular distribution of the laserball, extracted from the fit $\left(L_{i j}\right)$. 
Since the model is incomplete in SNOMAN (as of version 4_0091) and because the AV reflection study should remain as simple as possible, the default SNOMAN laserball was not used in the MC checks of Chapter 4.

\section{B.3.2 3D-PMT Model}

The use of the 3D-PMT model in SNOMAN provides the best response of the PMTs with their reflectors since it is likely that incoming photons reflect off the whole PMT assembly. The application of the data filters in Chapter 4 was made to get rid of the reflections caused by the PMT assembly. The reflections come approximately 20 ns after the PAVRs because they have an extra path length to go through in the $\mathrm{H}_{2} \mathrm{O}$ (between the AV and the PSUP). Like the PAVRs, their time differences to the prompt peak should vary with the position of the source. Most of the low hit PMTs (blue dots) in Figure 4.7 are probably due to those reflections that are not taken care of by the analytic model of Chapter 3 . Looking at the projection maps in this figure, there are fewer low reflected hits around the prompt hits, which clearly indicates that the light reflects with an important angle (not caused by the AV). The only exception is in Figure 4.8(b) where the PMT on the left at the back receives a lot of reflected light. This PMT passed the filters selection; the reflectors are like the $\mathrm{AV}$, characterized with reflection probabilities for both light polarizations in the 3D-PMT model [30]. This means that configuration $\mathrm{B}$ must have hit this PMT reflector with a high incident angle. Most PMT reflector reflected rays come out at $35^{\circ}$ from the normal. Further explanations are given in [1]. Reflections on PMT petals or other detector component would have to be identified as well, as it was done in this thesis, only if the width of the actual prompt time window was increased 
to include late light in the analysis of the optical data. Therefore, these reflections do not have to be considered as significant in the prompt light peak contamination since the present QOCA analysis only relies on prompt light. 


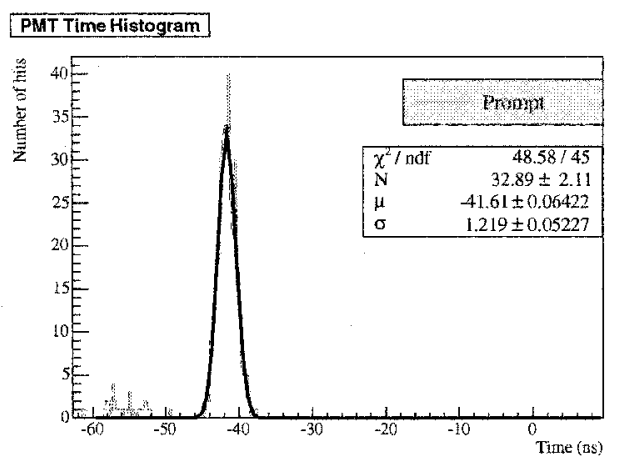

(a) Prompt (A) $R_{S}=475 \mathrm{~cm}$.

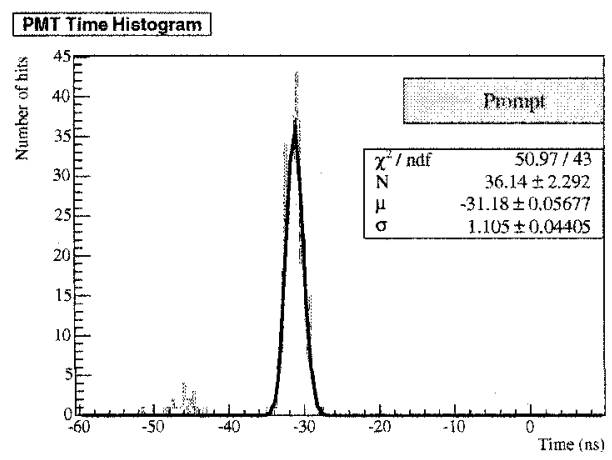

(c) Prompt (B) $R_{S}=550 \mathrm{~cm}$.

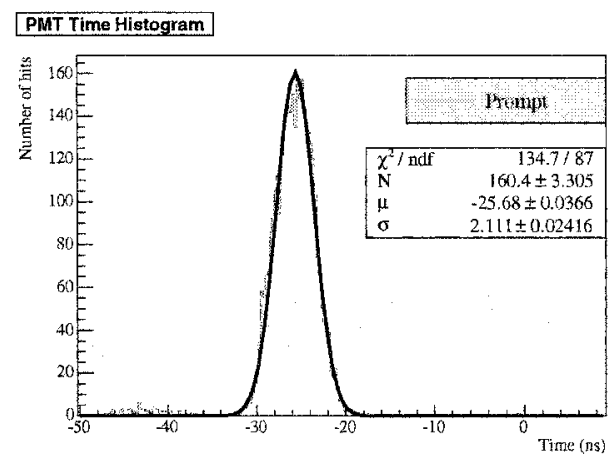

(e) Prompt (C) $R_{S}=575 \mathrm{~cm}$.

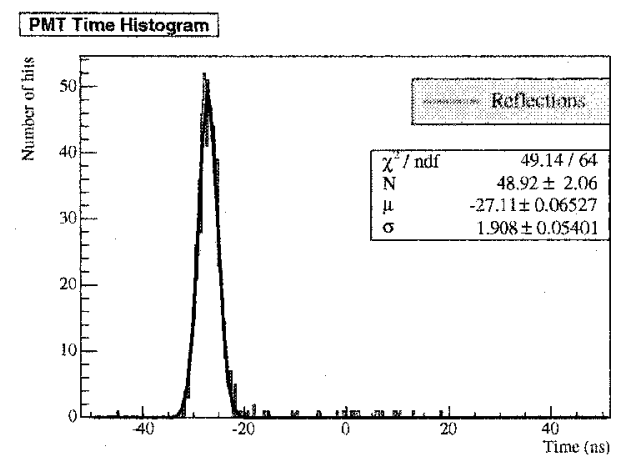

(b) Reflections (A) $R_{S}=475 \mathrm{~cm}$.

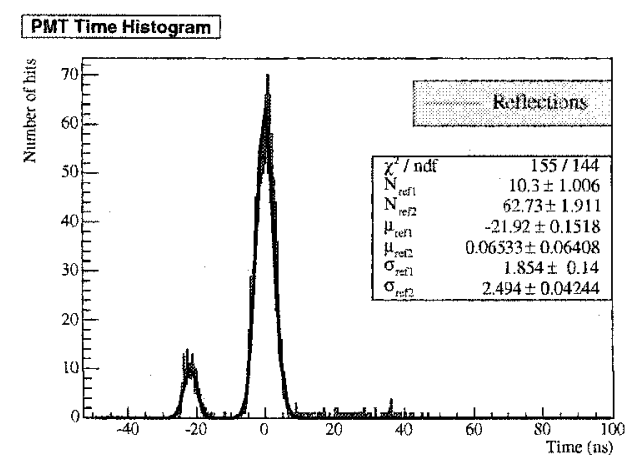

(d) Reflections (B) $R_{S}=550 \mathrm{~cm}$.

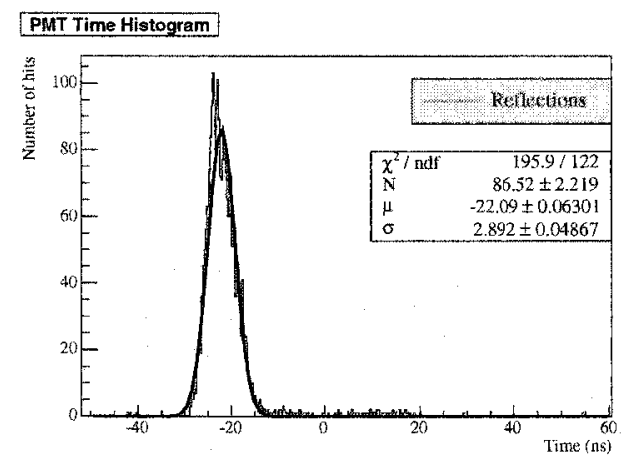

(f) Reflections (C) $R_{S}=575 \mathrm{~cm}$.

Figure B.4: Monte Carlo laserdisk source prompt and reflected light peak fits. The peaks are the same as in Figure B.3. The configuration is indicated between parentheses. 


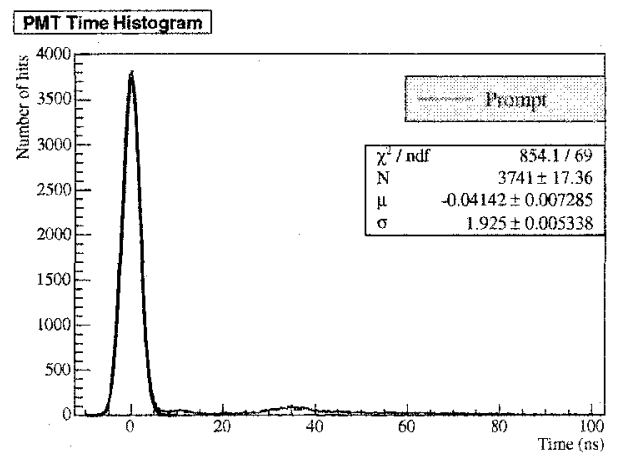

(a) Photon bomb (A) $R_{S}=475 \mathrm{~cm}$.

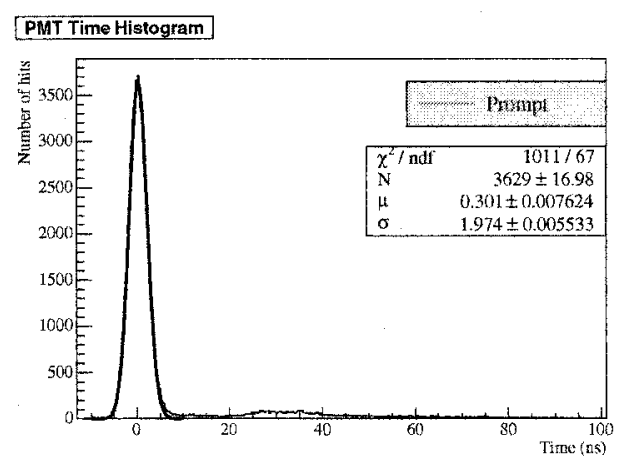

(c) Photon bomb (B) $R_{S}=550 \mathrm{~cm}$.

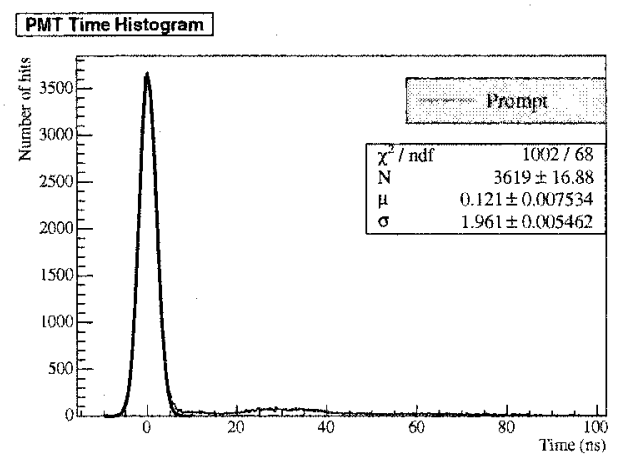

(e) Photon bomb (C) $R_{S}=575 \mathrm{~cm}$.

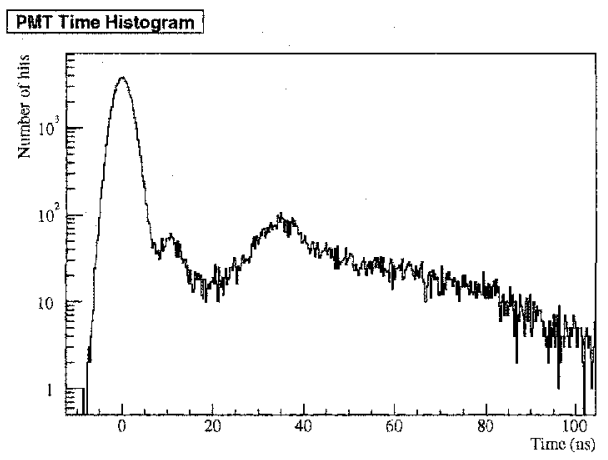

(b) Photon bomb (A) $R_{S}=475 \mathrm{~cm}$.

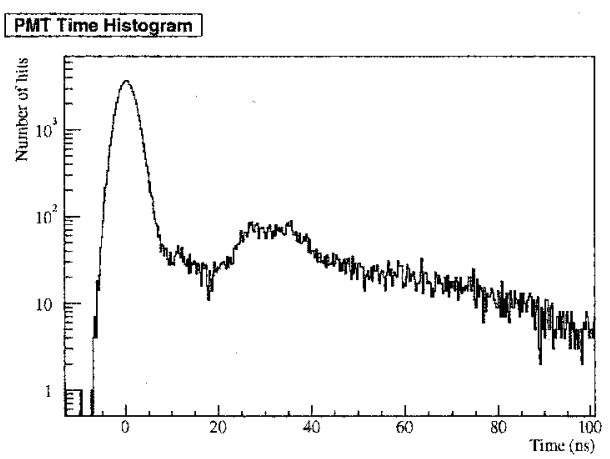

(d) Photon bomb (B) $R_{S}=550 \mathrm{~cm}$.

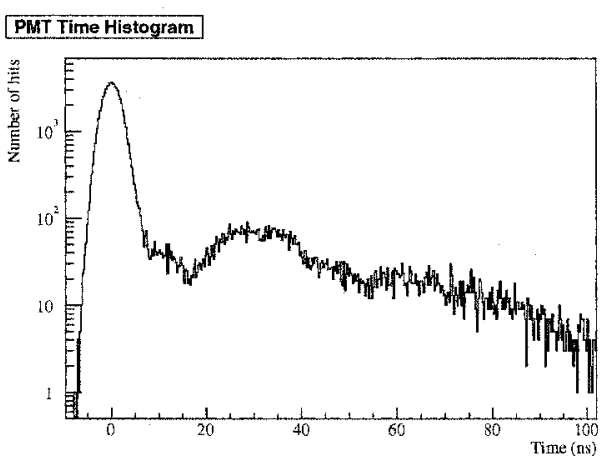

(f) Photon bomb (C) $R_{S}=575 \mathrm{~cm}$.

Figure B.5: Monte Carlo PMT time distributions for various photon bomb configurations. The configuration is indicated between parentheses. The logarithmic scale is also used to show the small amount of reflections compared to the fitted prompt light peak. The large $\chi^{2}$ values are due to that small amount of reflected light outside the prompt peak. 


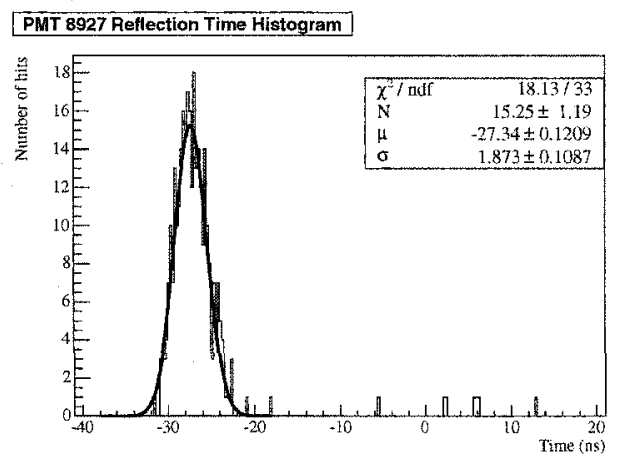

(a) Laserdisk (A) $R_{S}=475 \mathrm{~cm}$ PMT 8927.

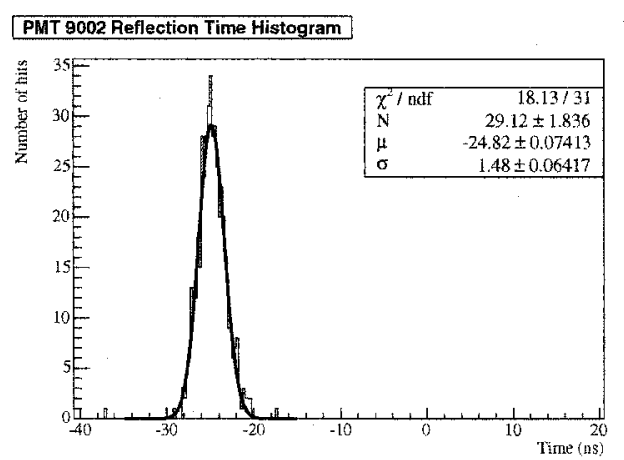

(c) Laserdisk (C) $R_{S}=575 \mathrm{~cm} \mathrm{PMT}$ 9002 .

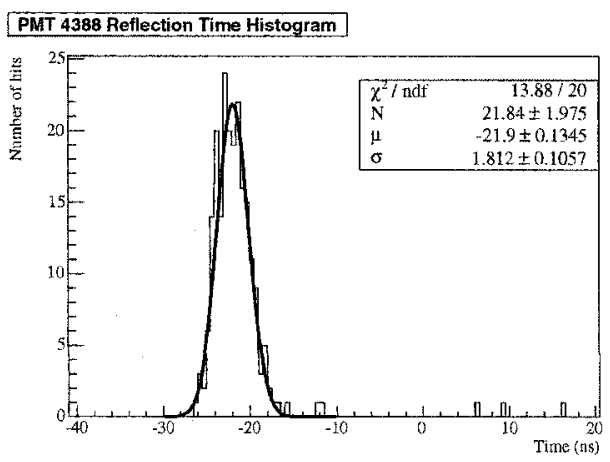

(b) Laserdisk (B) $R_{S}=550 \mathrm{~cm}$ PMT 4388.

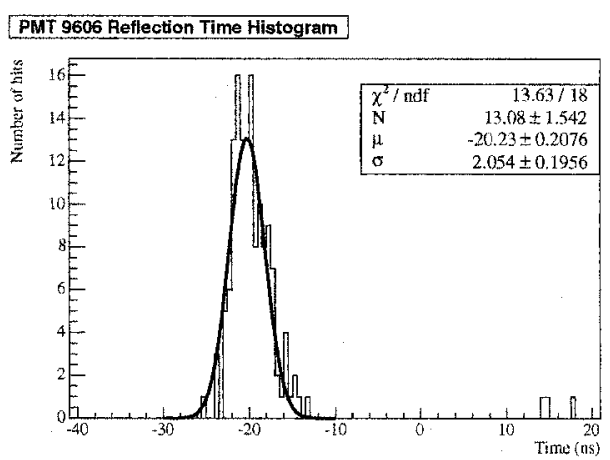

(d) Laserdisk (C) $R_{S}=575 \mathrm{~cm}$ PMT 9606.

Figure B.6: Monte Carlo PMT time distributions for various laserdisk configurations obtained from selected channels. These reflection peaks are extracted from the laserdisk time distributions of Figure B.4 by applying the filter 'Reflection' described in Table 4.2 to the specified PMT channel. 


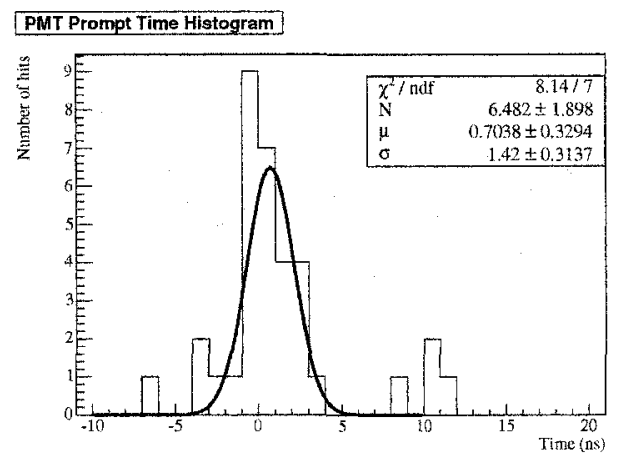

(a) Photon bomb (A) $R_{S}=475 \mathrm{~cm}$.

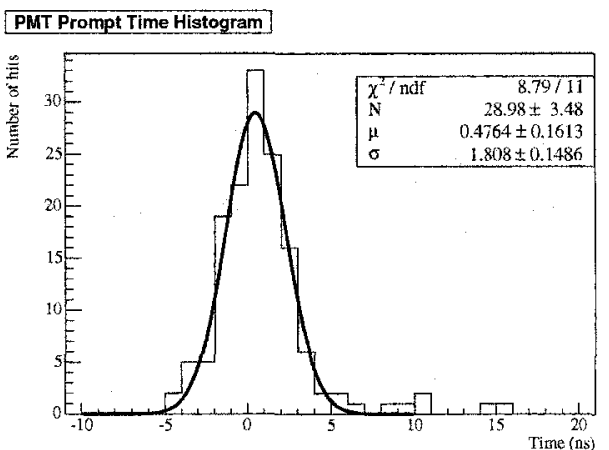

(b) Photon bomb (B) $R_{S}=550 \mathrm{~cm}$.

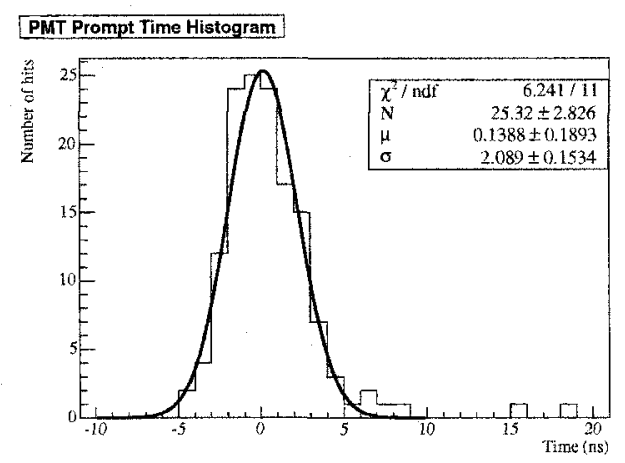

(c) Photon bomb (C) $R_{S}=575 \mathrm{~cm}$.

Figure B.7: Monte Carlo PMT time distributions for various photon bomb configurations obtained from a group of selected channels. These prompt peaks are extracted from the photon bomb time distributions of Figure B.5. It is obtained from keeping only $N_{T}$ PMTs to get the time difference $\left\langle\delta t_{\text {bomb }}\right\rangle$ of Equation (4.8). 


\section{Appendix C}

\section{Additional Fit Results}

To complete the study of the effects of using different subsets of runs and AV reflection cuts, an additional fit using the processing method with no PAVR cut was performed. This was done to verify if the prompt light contamination is real, but more importantly, to see how the fit handles it. There are here three different methods to process the data and two different ranges in radius, which gives six different fit results from the same scan. The fitted data subsets are summarized in Table C.1. As in Chapter 6, the $\mathrm{D}_{2} \mathrm{O}$ and $\mathrm{H}_{2} \mathrm{O}$ attenuations, and the PMTR are shown. All the plots of this appendix must be seen in color.

\section{C.1 Comparisons with Contaminated Data Fits}

The plots contained in this section are duplicated so that the effects of changing processing methods and using different data subsets can be isolated. The discussion that compares the fits obtained from the data processed with the standard and the enhanced methods was done in Chapter 6 . For the 'no cut' method, the cut for 


\begin{tabular}{|c|c|c|c|}
\hline Scan Acronym & Processing Method & PAVR Cut & $R_{S}^{\max }(\mathrm{cm})$ \\
\hline $\operatorname{oct} 03$ & Standard & $85^{\circ}$ (default) & 500 \\
\hline oct03nc & No cut & None & 500 \\
\hline oct03new & Enhanced & $R_{S^{-}}$dependent (new) & 500 \\
\hline oct03h & $\begin{array}{l}\text { Standard with more } \\
\text { high radius runs }\end{array}$ & $85^{\circ}$ (default) & 555 \\
\hline oct03hne & $\begin{array}{l}\text { No cut with more } \\
\text { high radius runs }\end{array}$ & None & 555 \\
\hline oct03hnew & $\begin{array}{l}\text { Enhanced with more } \\
\text { high radius runs }\end{array}$ & $R_{S}$-dependent (new) & 555 \\
\hline
\end{tabular}

Table C.1: More processing methods for the October 2003 scan.

PAVRs was simply eliminated, leaving the PAVRs to freely contaminate the prompt peak light. Like the other methods, it was used with $R_{S}^{\max }=500 \mathrm{~cm}$ runs and then with high radius runs with $R_{S}^{\max }=555 \mathrm{~cm}$.

\section{C.1.1 Media Attenuations}

Figure C.1 shows the media attenuation coefficients for all the fits with different subsets of runs. Compared to the standard or the enhanced processing methods, which both have a PAVR cut, the $\mathrm{D}_{2} \mathrm{O}$ attenuation decreases and the $\mathrm{H}_{2} \mathrm{O}$ attenuation increases for all wavelengths when no PAVR cut is applied. Figures C.1(a) and C.1(b) show that the effect is not important when $R_{S} \leqslant 500 \mathrm{~cm}$ which tells that the reflected light is not very intense. This is due to the low reflection probability found from low incident angles on the AV. Figures C.1(c) and C.1(d) show that the effect is more important when high radius runs are all analyzed. In this case, the reflection probability can be high and some PAVRs will really affect the prompt light 


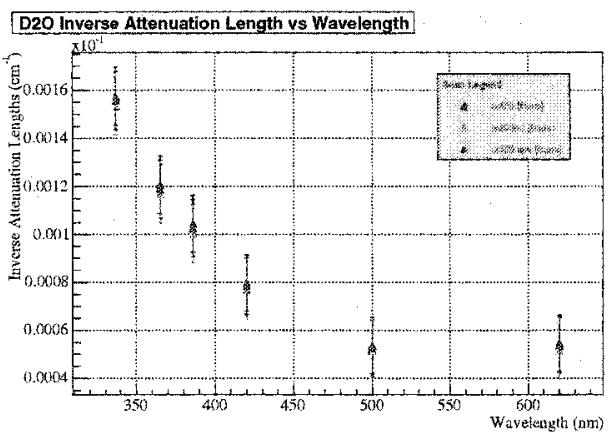

(a) $\mathrm{D}_{2} \mathrm{O}$ with $R_{S}^{\max }=500 \mathrm{~cm}$.

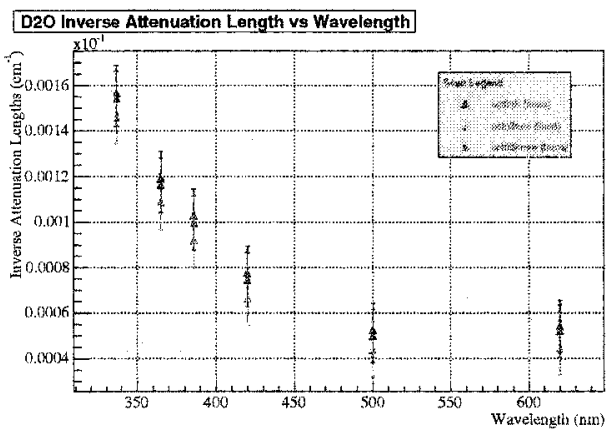

(c) $\mathrm{D}_{2} \mathrm{O}$ with $R_{S}^{\max }=555 \mathrm{~cm}$.

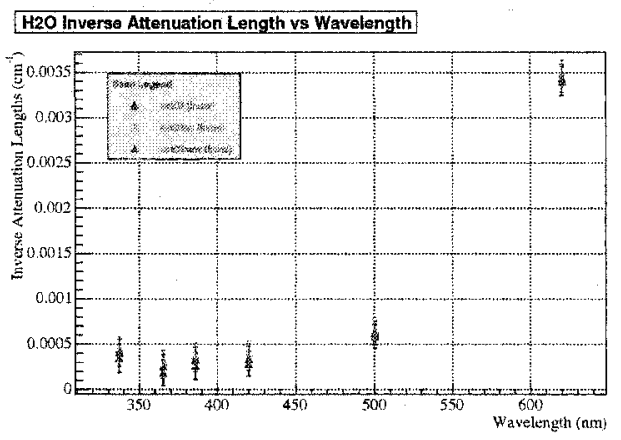

(b) $\mathrm{H}_{2} \mathrm{O}$ with $R_{S}^{\max }=500 \mathrm{~cm}$.

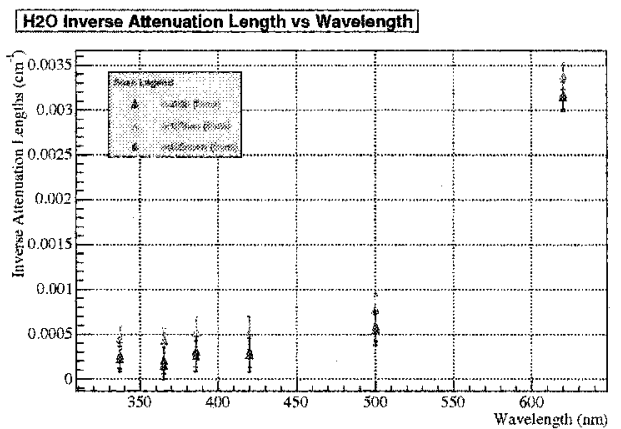

(d) $\mathrm{H}_{2} \mathrm{O}$ with $R_{S}^{\max }=555 \mathrm{~cm}$.

Figure C.1: $\mathrm{D}_{2} \mathrm{O}$ and $\mathrm{H}_{2} \mathrm{O}$ attenuations obtained from runs with $\mathrm{R}_{S}^{\max }=500$ and $555 \mathrm{~cm}$.

occupancy. The $\mathrm{D}_{2} \mathrm{O}$ attenuation decreases because more light is detected coming from the central volume. Inversely, the $\mathrm{H}_{2} \mathrm{O}$ attenuation increases as if the light was blocked by the $\mathrm{AV}-\mathrm{H}_{2} \mathrm{O}$ layer in front of the PMTs.

Figure C.2 shows the same results but rearranged by processing methods. Figures C.2(a) and C.2(b) show that the addition of high radius runs combined to the use of the standard processing method does not affect much the attenuations. The reason was shown to be that the method is too restrictive when eliminating PMTs. Figures C.2(c) and C.2(d) show that the addition of high radius runs combined using no PAVR cut does affect significantly the attenuations as explained before. 


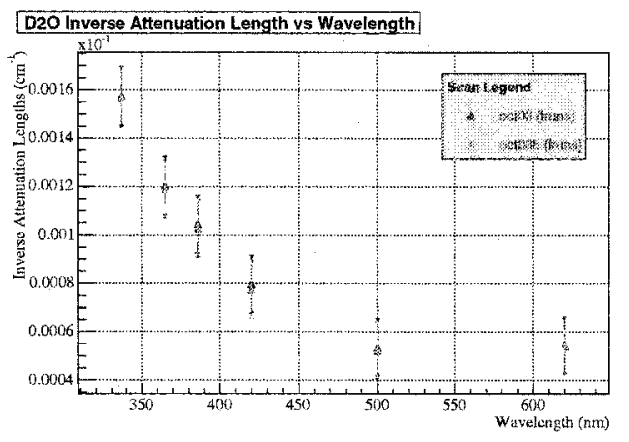

(a) $\mathrm{D}_{2} \mathrm{O}$ using the standard method.

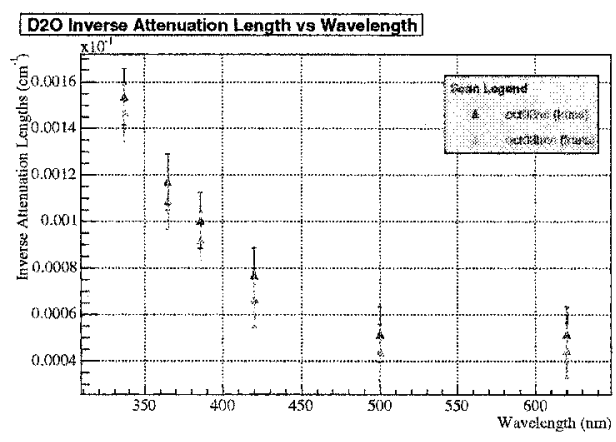

(c) $\mathrm{D}_{2} \mathrm{O}$ with no PAVR cut.

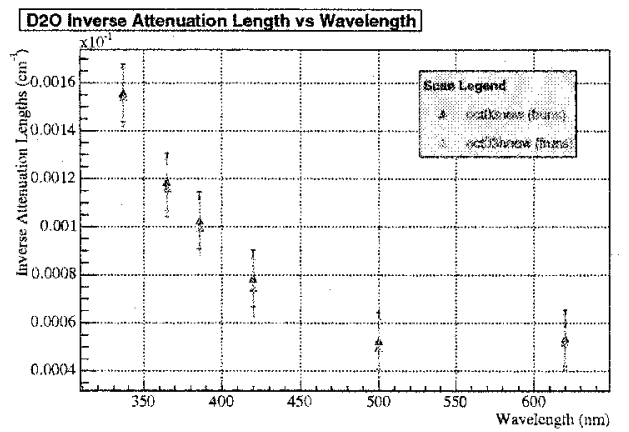

(e) $\mathrm{D}_{2} \mathrm{O}$ using the enhanced method.

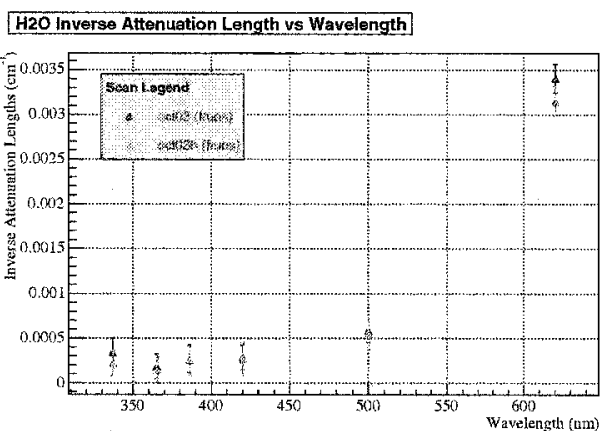

(b) $\mathrm{H}_{2} \mathrm{O}$ using the standard method.

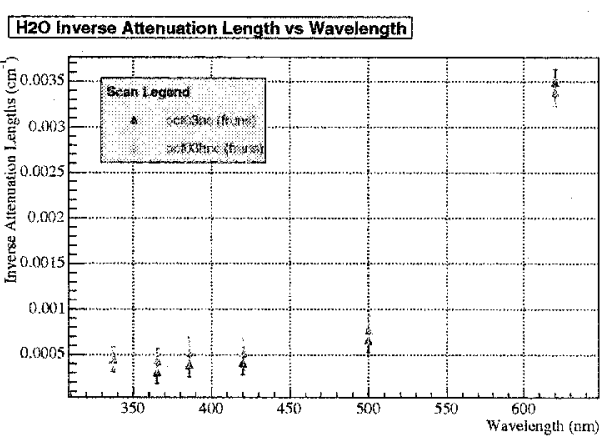

(d) $\mathrm{H}_{2} \mathrm{O}$ with no PAVR cut.

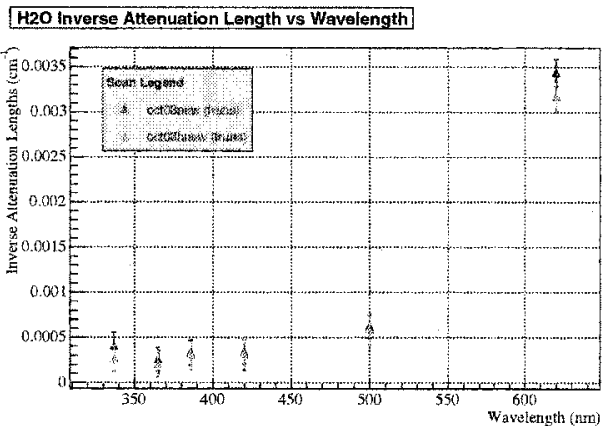

(f) $\mathrm{H}_{2} \mathrm{O}$ using the enhanced method.

Figure C.2: Effect of adding high radius runs on the $\mathrm{D}_{2} \mathrm{O}$ and $\mathrm{H}_{2} \mathrm{O}$ attenuations when using various processing methods. 
Figures C.2(e) and C.2(f) show that the addition of high radius runs combined to the use of the enhanced processing method does not affect much the attenuations since the method eliminates the reflections properly. Hence, the AV reflection cut is important to get the correct media attenuations in the whole $\mathrm{D}_{2} \mathrm{O}$ volume since the attenuations are sensitive to the reflections when high radius runs are analyzed.

\section{C.1.2 PMT Response}

Figures C.3 and C. 4 show the PMTR for all the fits for the six wavelengths. In Figure C.3, the PMTR results seem consistent. The PMTR between $\left[10^{\circ}, 40^{\circ}\right]$ slightly increases compared to the standard and enhanced methods for $R_{S}^{\max }=500$ $\mathrm{cm}$ when no PAVR cut is applied. The reflections do not affect much the response returned by the fit. This confirms what was found for the attenuations: the amount of reflected light is low when $R_{S}^{\max }=500 \mathrm{~cm}$. In Figure C.4, the PMTR varies a lot when no PAVR cut is applied. The PMTR between in the range $\left[10^{\circ}, 40^{\circ}\right]$ is more affected when high radius runs are included. It confirms that reflections do not reach PMTs at normal $\left(0^{\circ}\right)$ or high incidence. Beyond $40^{\circ}$, the effect is not seen since the light detected at these angles is mostly transmitted light.

Figures C.5, C.6, and C.7 show the effect on the PMTR when adding high radius runs for the three processing methods. As for the media attenuations, the results shown in Figures C.5 and C.7 are consistent for the standard and the enhanced methods. The variations at high incident angles come from sampling more PMTs. The effect of adding high radius runs seems to be the same for both the standard and the enhanced processing methods, showing that the PMT variability produces variations in a consistent way. Figure C.6 emphasizes the effect of the reflections. 
Hence, the AV reflection cut is very important to get the correct PMTR in a wide range of incident angle since the response of the PMTs is sensitive to the reflections from $\theta_{P M T}=10^{\circ}$ and above. Figure C.8 gives the PMTR comparison that was given in Chapter 6 but for all the wavelengths.

\section{C.1.3 Summary}

Table C.2 summarizes the variations of the optical parameters observed in this thesis using different processing methods given a similar data set. The increase or decrease observed always depend on three things: the AV reflections, the detector volume sampled, and the number of PMTs. The media attenuations are influenced by the reflections and the radius of the volume sampled. The PMTR is varying with the reflections and the number of PMTs sampled. For both, the variations can always be explained by two reasons. Hence, when the variations are small or when two fits are consistent, it can be caused by the counterbalance of two effects. 


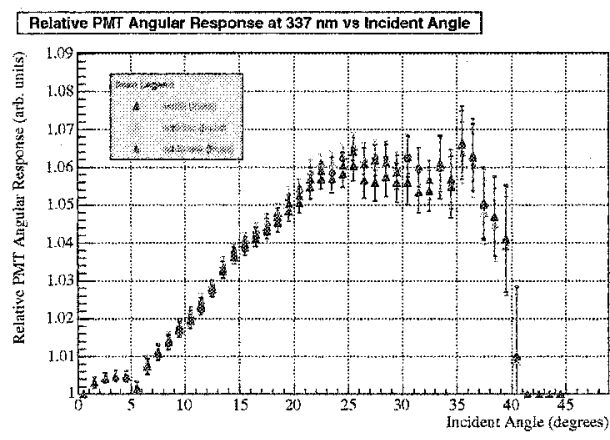

(a) PMTR at $337 \mathrm{~nm}$.

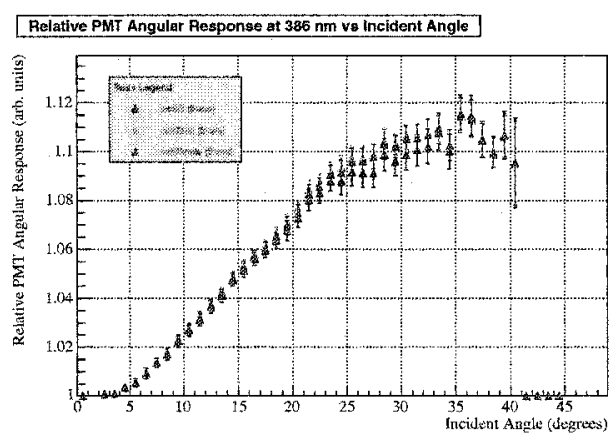

(c) PMTR at $386 \mathrm{~nm}$.

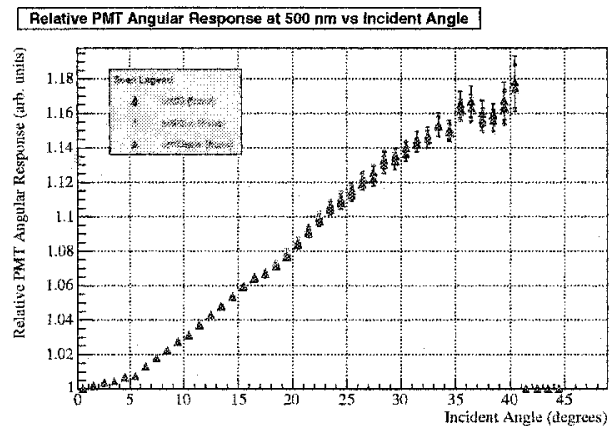

(e) PMTR at $500 \mathrm{~nm}$.

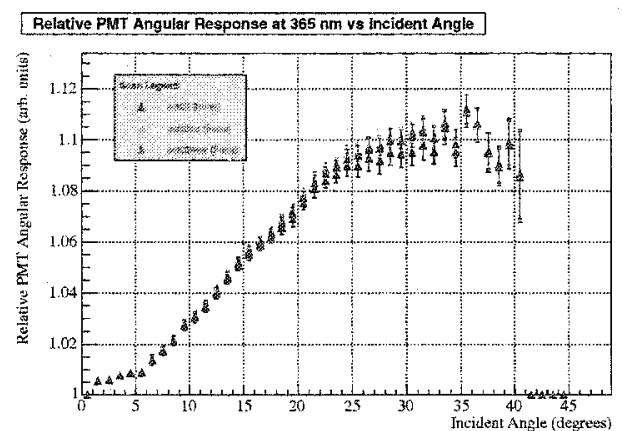

(b) PM'TR at $365 \mathrm{~nm}$.

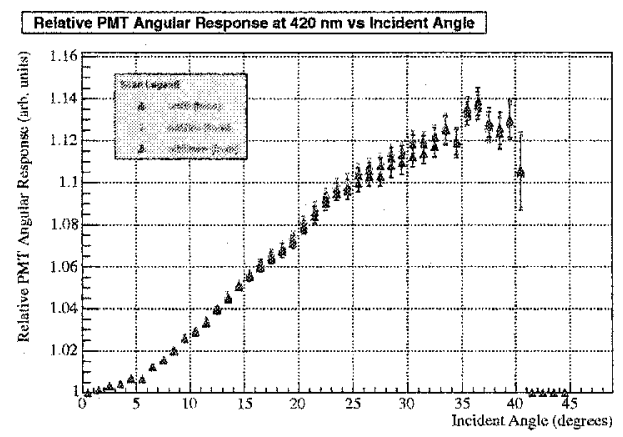

(d) PMTR at $420 \mathrm{~nm}$.

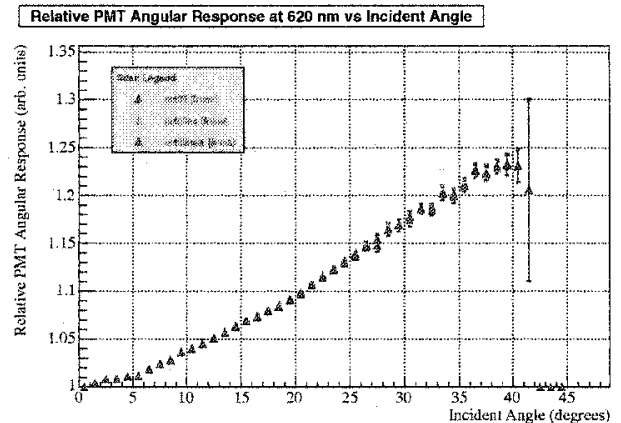

(f) PMTR at $620 \mathrm{~nm}$.

Figure C.3: Relative PMT angular response obtained from runs with $R_{S}^{\max }=500$ $\mathrm{cm}$. Note that the vertical scale is different for each wavelength. 


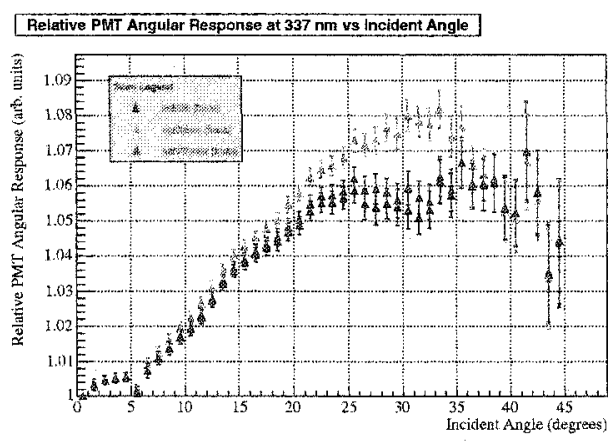

(a) PMTR at $337 \mathrm{~nm}$.

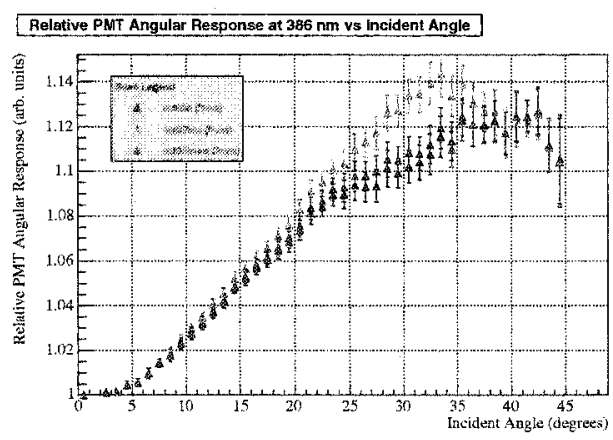

(c) PMTR at $386 \mathrm{~nm}$.

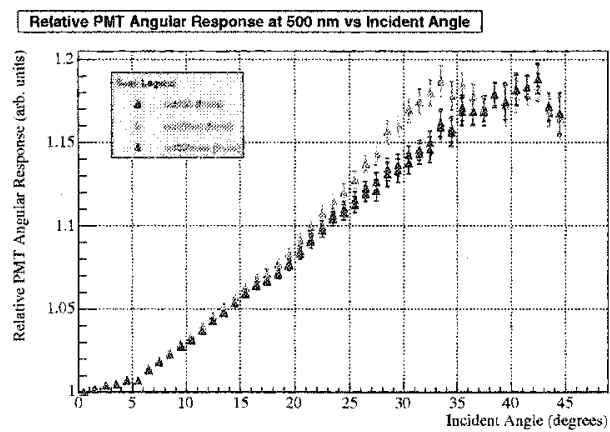

(e) PMTR at $500 \mathrm{~nm}$.

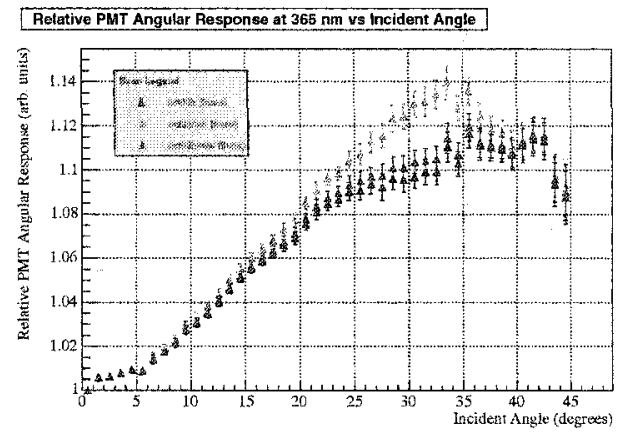

(b) PMTR at $365 \mathrm{~nm}$.

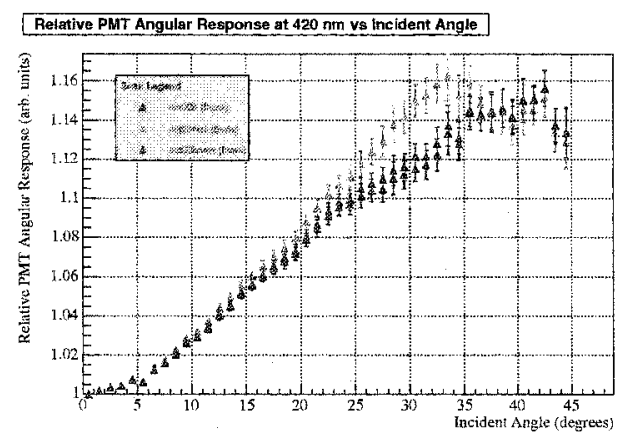

(d) PMTR at $420 \mathrm{~nm}$.

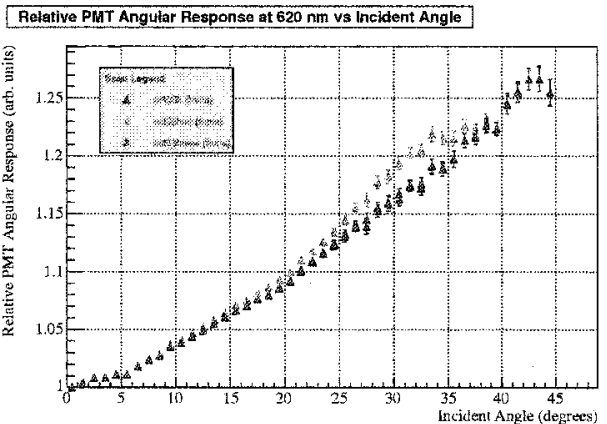

(f) PMTR at $620 \mathrm{~nm}$.

Figure C.4: Relative PMT angular response obtained from runs with $R_{S}^{\max }=555$ $\mathrm{cm}$. Note that the vertical scale is different for each wavelength. 


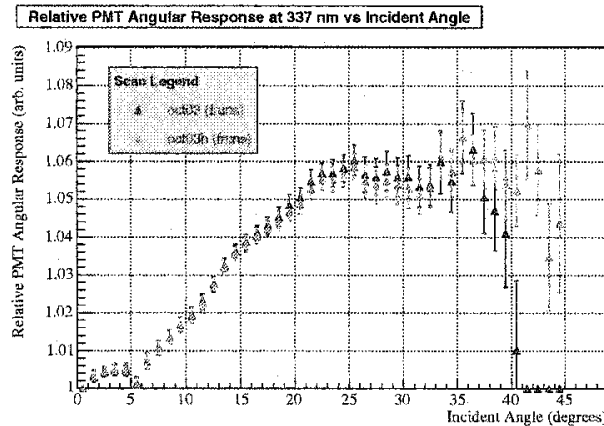

(a) PMTR at $337 \mathrm{~nm}$.

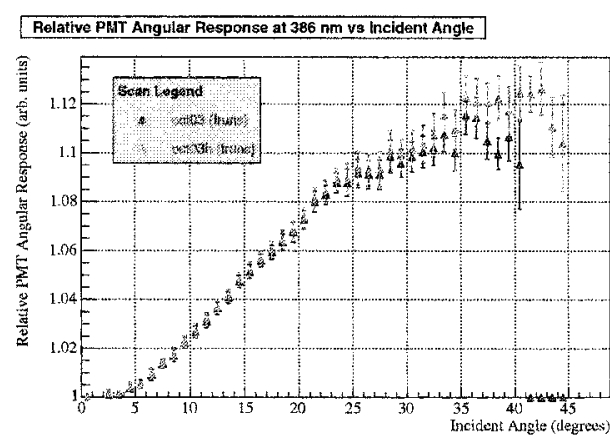

(c) PMTR at $386 \mathrm{~nm}$.

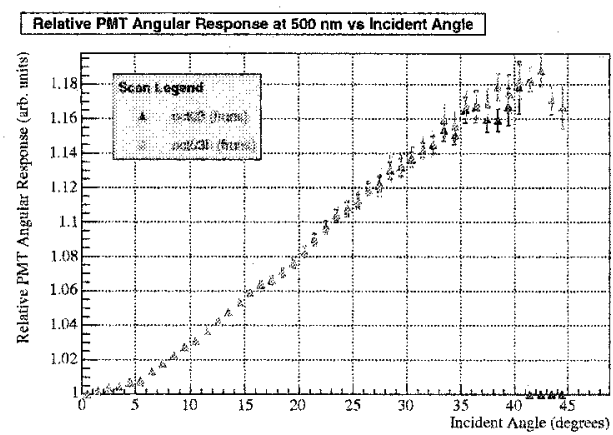

(e) PMTR at $500 \mathrm{~nm}$.

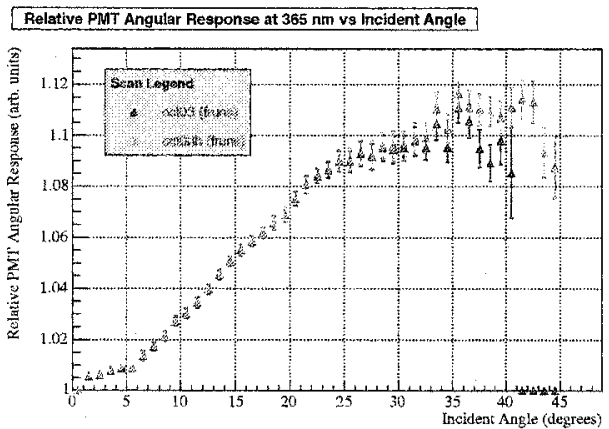

(b) PMTR at $365 \mathrm{~nm}$.

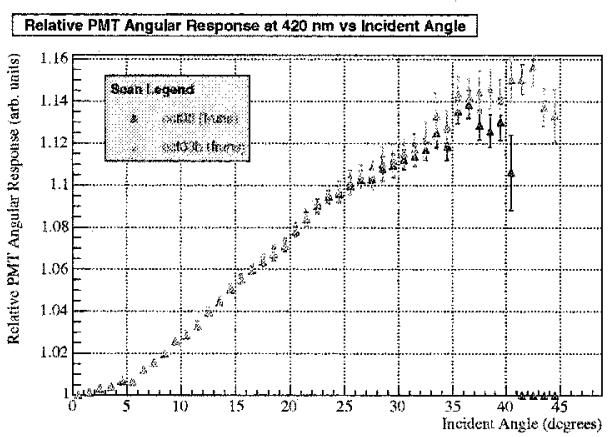

(d) PMTR at $420 \mathrm{~nm}$.

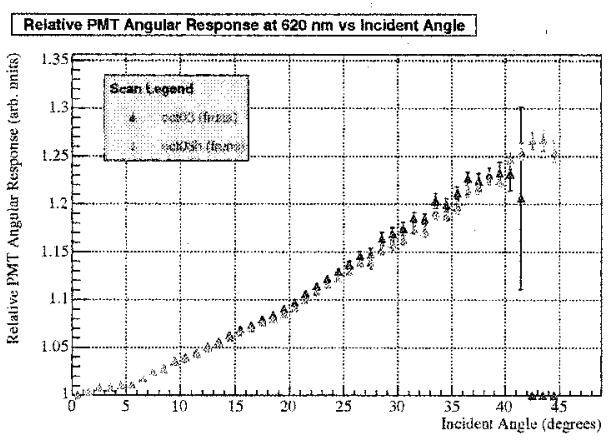

(f) PMTR at $620 \mathrm{~nm}$.

Figure C.5: Effect of adding high radius runs on the relative PMT angular response when using the standard processing method. Note that the vertical scale is different for each wavelength. 


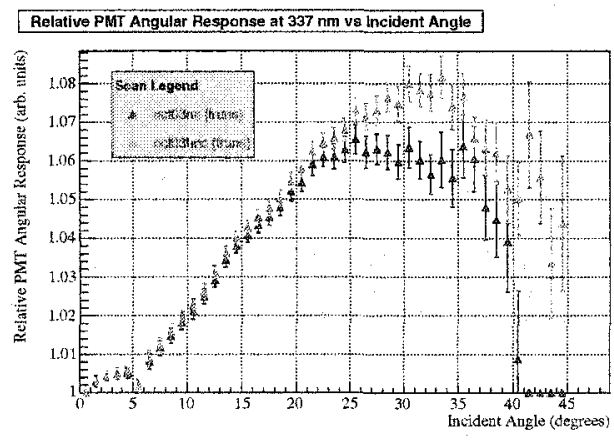

(a) PMTR at $337 \mathrm{~nm}$.

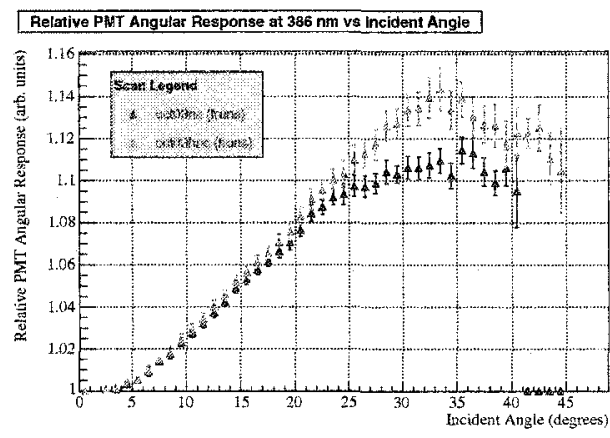

(c) PMTR at $386 \mathrm{~nm}$.

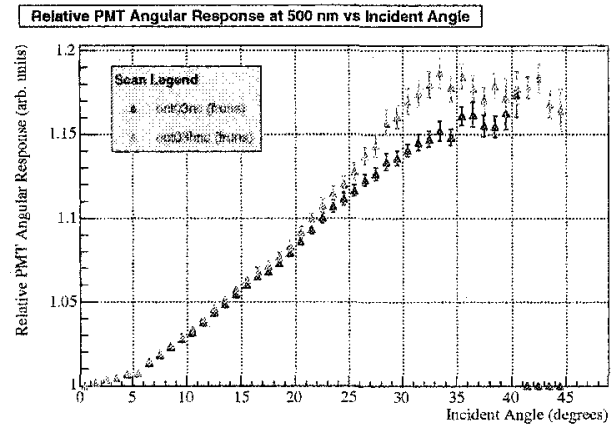

(e) PMTR at $500 \mathrm{~nm}$.

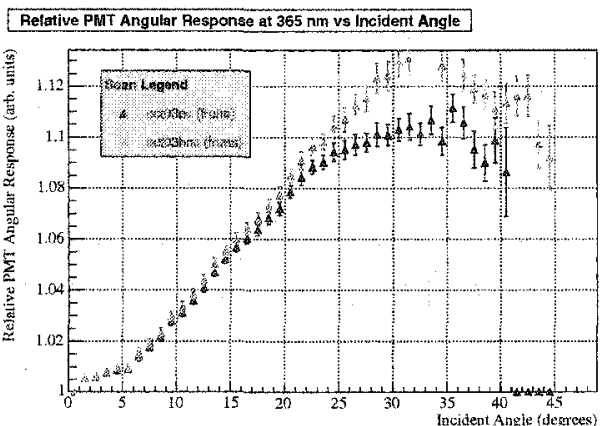

(b) PMTR at $365 \mathrm{~nm}$.

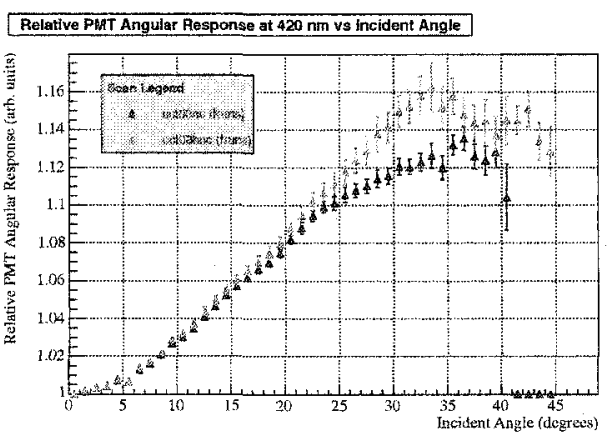

(d) PMTR at $420 \mathrm{~nm}$.

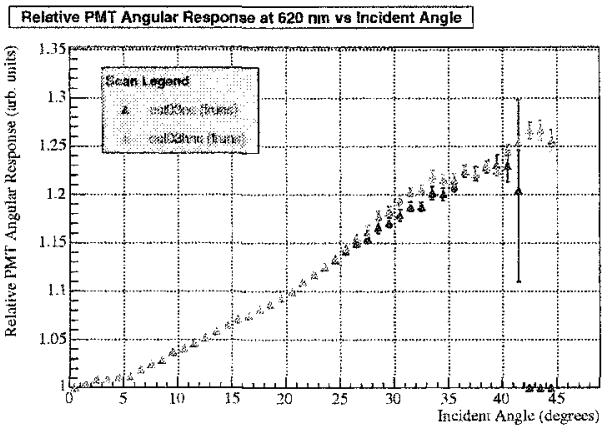

(f) PMTR at $620 \mathrm{~nm}$.

Figure C.6: Effect of adding high radius runs on the relative PMT angular response when no cut is applied for AV reflections. Note that the vertical scale is different for each wavelength. 


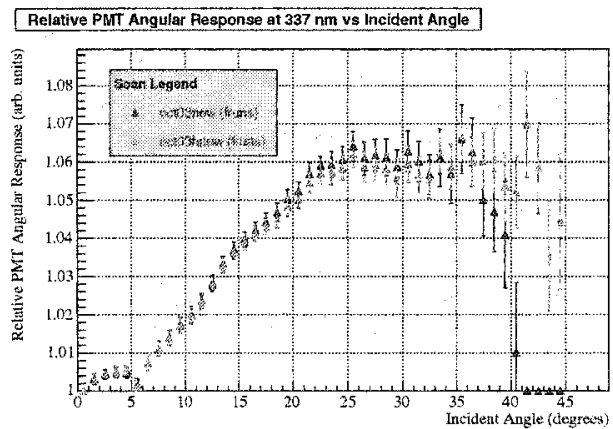

(a) PMTR at $337 \mathrm{~nm}$.

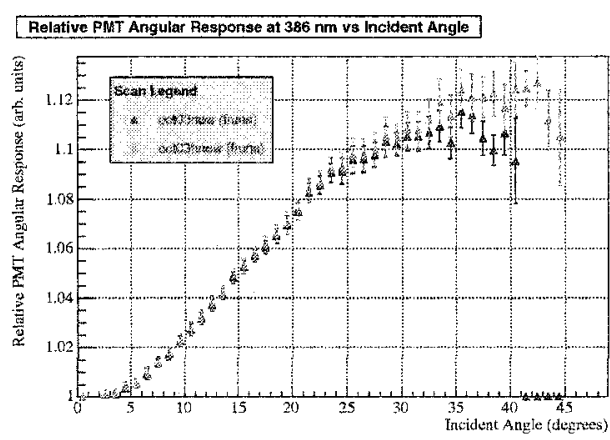

(c) PMTR at $386 \mathrm{~nm}$.

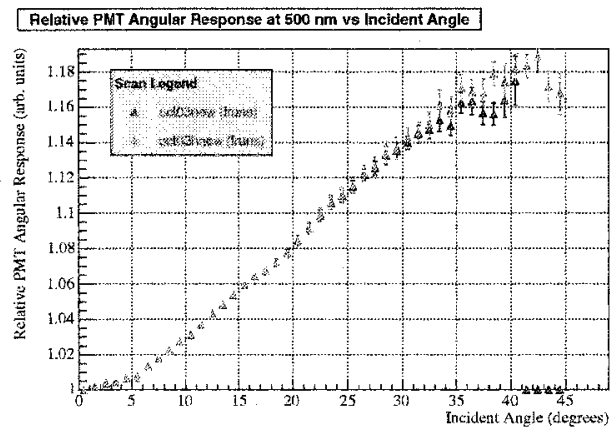

(e) PMTR at $500 \mathrm{~nm}$.

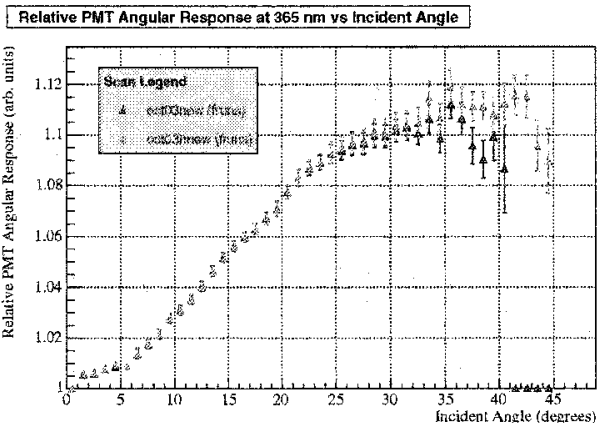

(b) PMTR at $365 \mathrm{~nm}$.

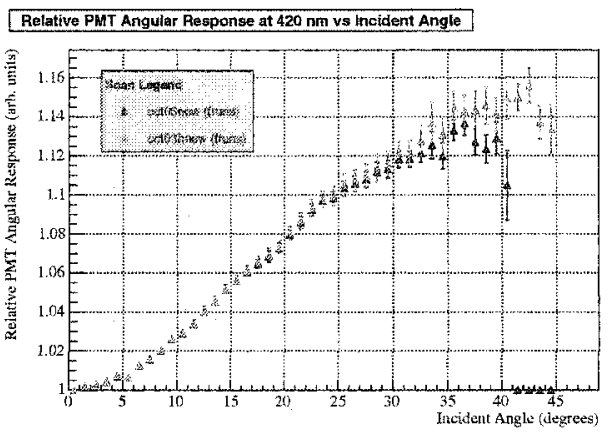

(d) PMTR at $420 \mathrm{~nm}$.

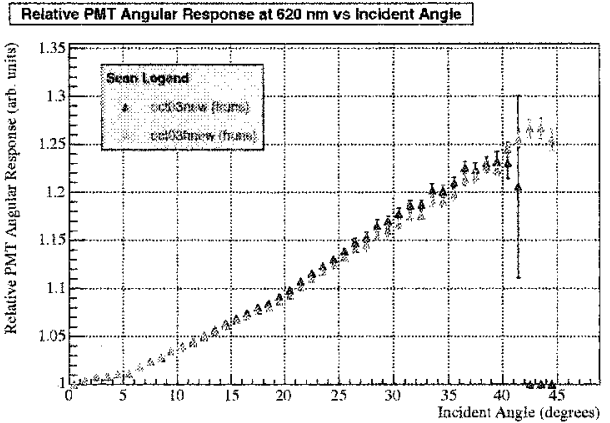

(f) PMTR at $620 \mathrm{~nm}$.

Figure $\boldsymbol{C . 7 : ~ E f f e c t ~ o f ~ a d d i n g ~ h i g h ~ r a d i u s ~ r u n s ~ o n ~ t h e ~ r e l a t i v e ~ P M T ~ a n g u l a r ~ r e s p o n s e ~}$ when using the enhanced processing method. Note that the vertical scale is different for each wavelength. 


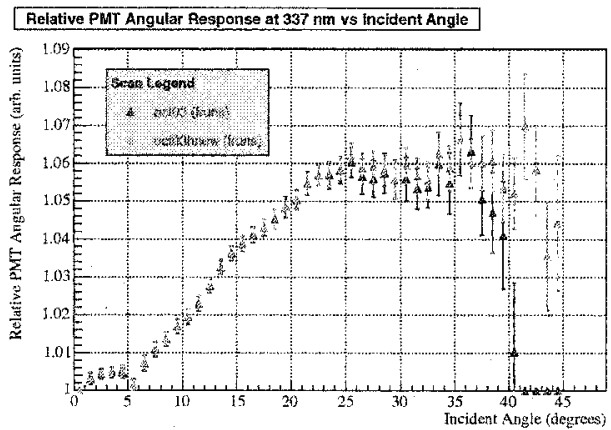

(a) PMTR at $337 \mathrm{~nm}$.

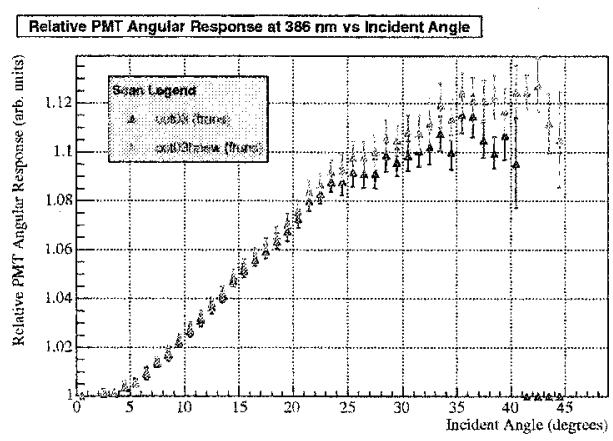

(c) PMTR at $386 \mathrm{~nm}$.

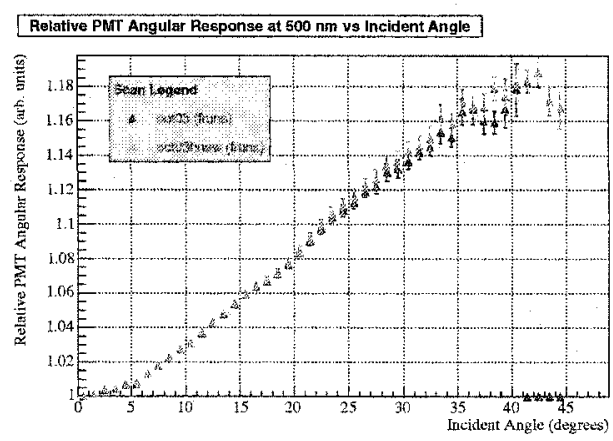

(e) PMTR at $500 \mathrm{~nm}$.

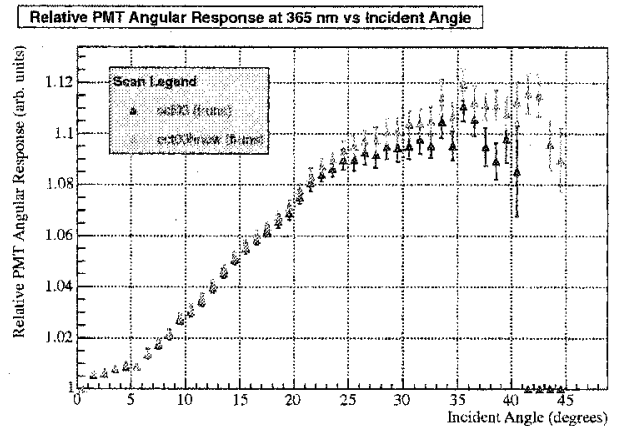

(b) PMTR at $365 \mathrm{~nm}$.

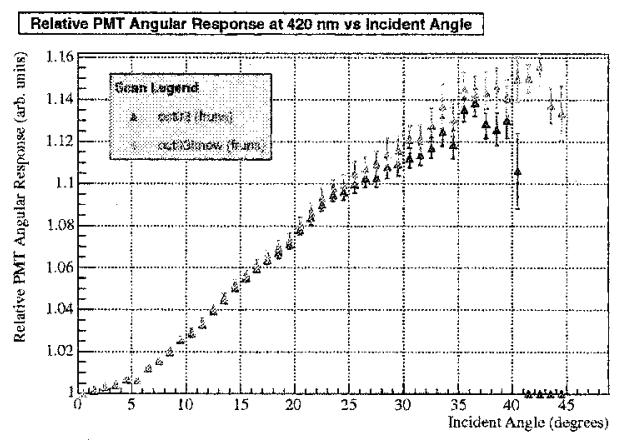

(d) PMTR at $420 \mathrm{~nm}$.

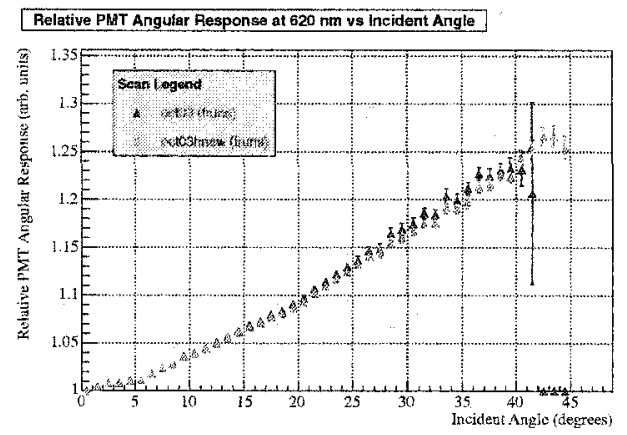

(f) PMTR at $620 \mathrm{~nm}$.

Figure C.8: Net effect of adding high radius runs and using the enhanced processing method on the relative PMT angular response. Note that the vertical scale is different for each wavelength. 


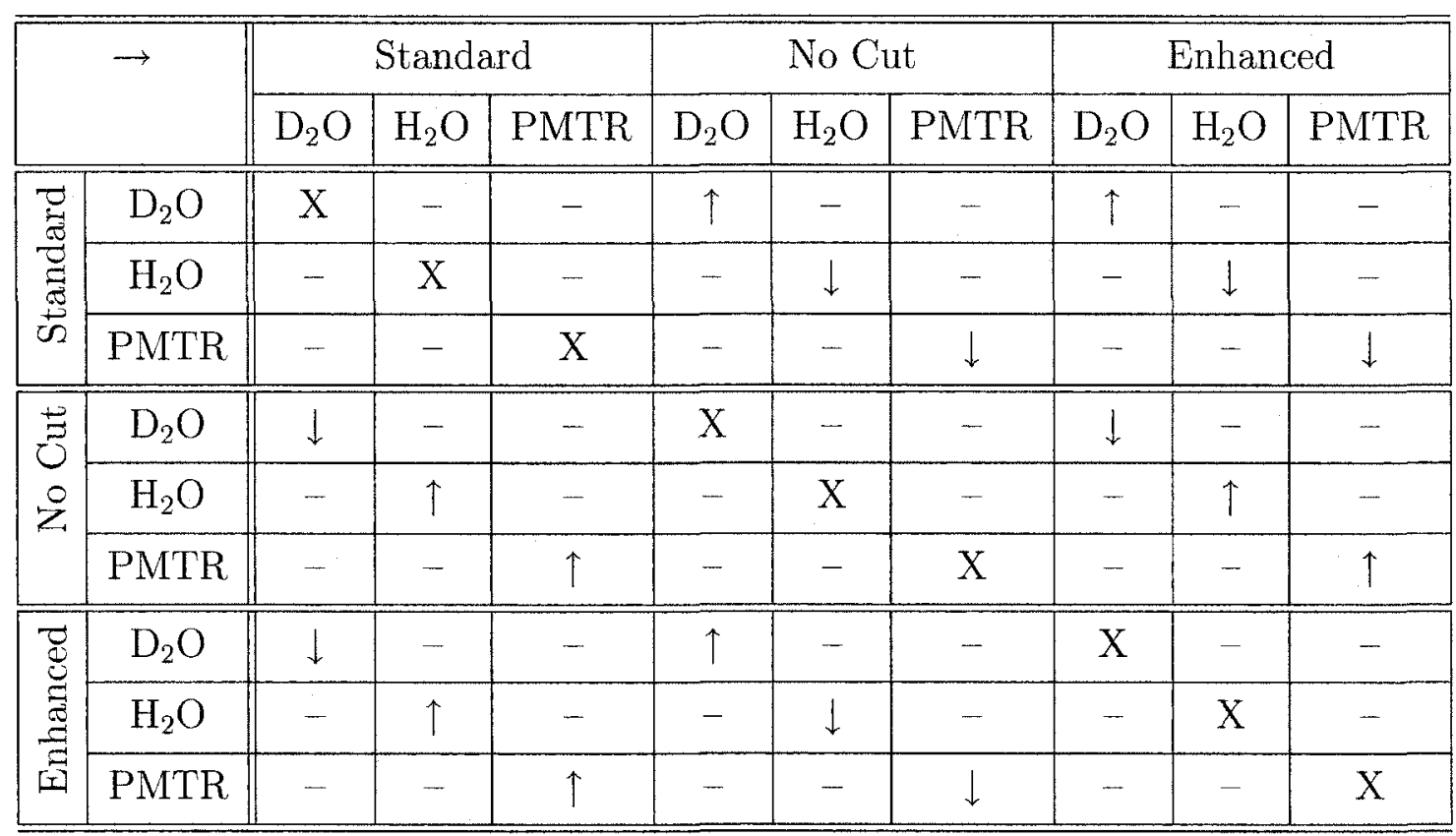

Table C.2: Summary of the optical parameters variations with respect to the processing method used. $\mathrm{D}_{2} \mathrm{O}$ and $\mathrm{H}_{2} \mathrm{O}$ refer to the media attenuations, PMTR to the PMT angular response. The PMTR comparison is made in the range $10^{\circ}-40^{\circ}$. The comparison is made from left to right: the parameter in the left part increases $(\uparrow)$ or decreases $(\downarrow)$ compared to the parameter in the right part of the table. The comparison is not applicable for bins with dashes (-) and unnecessary for bins with Xs. Note that the these variations are specific to the October 2003 scan data. 


\section{Bibliography}

[1] B. A. Moffat, The Optical Calibration of the Sudbury Neutrino Observatory, $\mathrm{PhD}$ thesis, Queen's University, Kingston, Canada, 2001.

[2] SNO Collaboration, Q. Ahmad et al., Measurements of charged current interactions produced by ${ }^{8} B$ solar neutrinos at the Sudbury Neutrino Observatory, Phys. Rev. Lett. 87, 071301, SNO first results, June 2001.

[3] R. Fernow, Introduction to Experimental Particle Physics (Cambridge University Press, 1986).

[4] J. D. Jackson, Classical Electrodynamics, Third ed. (John Wiley and Sons, 1999).

[5] R. J. Ford, Nitrogen/dye laser system for the optical calibration of SNO, Master's thesis, Queen's University, Kingston, Canada, 1993.

[6] R. J. Ford, SNO $N_{2} /$ Dye Laser and Fibre Optic Diffuser Ball System Manual, SNO-STR-96-056, 1997. 
[7] D. R. Grant, Optical Calibration of the Sudbury Neutrino Observatory and Determination of the ${ }^{8} B$ Solar Neutrino Flux in the Salt Phase, PhD thesis, Carleton University, Ottawa, Canada, 2004.

[8] C. V. Ouellet, A Study of Relative Efficiencies of Photomultiplier Tubes in the Sudbury Neutrino Observatory, Master's thesis, Queen's University, Kingston, Canada, 2003.

[9] C. Jillings, Multiple photo-electron effects in optical calibration, SNO Internal Report, 1999.

[10] J. C. Maneira, PMT variability with incidence angle in salt optics, SNO Internal Report, October 31, 2003.

[11] SNO Collaboration, QSNO Official Web Page, http://owl.phy.queensu.ca/private/qsno/, 2004.

[12] SNO Collaboration, The SNOMAN User Manual, SNO Collaboration internal reference manual updated periodically, 2004.

[13] R. Brun and F. Rademakers, ROOT - An object oriented data analysis framework, Nuclear Instuments and Methods A, http://root.cern.ch, 1997.

[14] P. Harvey, XSnoed Official Web Page, http://manhattan.sno.laurentian.ca/xsnoed/, 2001.

[15] M. S. Neubauer, Evidence for Electron Neutrino Flavor Change Through Measurement of the ${ }^{8} B$ Solar Neutrino Flux at the Sudbury Neutrino Observatory, PhD thesis, University of Pennsylvania, Philadelphia, USA, 2001. 
[16] E. Hecht, Optics, Third ed. (Addison Wesley, 1998).

[17] SNO Collaboration, The SNOMAN Companion, Online web page tool, http://www.physics.carleton.ca/research/sno/anal/doc/html/companion.html, 2004.

[18] S. Biller et al., Optics6b, SNO Internal Report, April 9, 2001.

[19] R. S. Dosanjh, Private communication, January 2004.

[20] R. S. Dosanjh, Salt Phase Optics Extraction, SNO Internal Report, May 2003.

[21] R. S. Dosanjh and D. R. Grant, Optics During the Salt Phase of SNO, SNO Internal Report, April 2002.

[22] R. S. Dosanjh, Laserball miniscans, SNO Internal Report, May 2002.

[23] M. D. Lay, Creation and Detection of Čerenkov Light in the Sudbury Neutrino Observatory, PhD thesis, Oxford University, Oxford, United Kingdom, 1994.

[24] J. C. Maneira, First laserball run in the NCD phase: preliminary plots, SNO Internal Report, June 11, 2004.

[25] J. C. Maneira, Laserball Deployment Plan Proposal for NCD Phase, SNO Internal Report, February 12, 2004.

[26] J. C. Maneira, Private communication, First NCD optical scan, July 5, 2004.

[27] D. R. Grant, Private communication, December 2003.

[28] J. Heise, Unusual SNO Objects, SNO Internal Report, December 20, 2003. 
[29] F. Duncan, Laserball Shadowing, SNO Internal Report, 2000.

[30] C. Krauss, Private communication, SNO Analysis Collaboration Meeting, February 2004. 